TRANSACTIONS OF THE

AMERICAN MATHEMATICAL SOCIETY

Volume 364, Number 3, March 2012, Pages 1225-1279

S 0002-9947(2011)05342-0

Article electronically published on October 17, 2011

\title{
QUANTUM DOUBLE OF HOPF MONADS AND CATEGORICAL CENTERS
}

\author{
ALAIN BRUGUIÈRES AND ALEXIS VIRELIZIER
}

\begin{abstract}
The center $\mathcal{Z}(\mathcal{C})$ of an autonomous category $\mathcal{C}$ is monadic over $\mathcal{C}$ (if certain coends exist in $\mathcal{C}$ ). The notion of a Hopf monad naturally arises if one tries to reconstruct the structure of $\mathcal{Z}(\mathcal{C})$ in terms of its monad $Z$ : we show that $Z$ is a quasitriangular Hopf monad on $\mathcal{C}$ and $\mathcal{Z}(\mathcal{C})$ is isomorphic to the braided category $Z-\mathcal{C}$ of $Z$-modules. More generally, let $T$ be a Hopf monad on an autonomous category $\mathcal{C}$. We construct a Hopf monad $Z_{T}$ on $\mathcal{C}$, the centralizer of $T$, and a canonical distributive law $\Omega: T Z_{T} \rightarrow Z_{T} T$. By Beck's theory, this has two consequences. On one hand, $D_{T}=Z_{T} \circ_{\Omega} T$ is a quasitriangular Hopf monad on $\mathcal{C}$, called the double of $T$, and $\mathcal{Z}(T-\mathcal{C}) \simeq D_{T}-\mathcal{C}$ as braided categories. As an illustration, we define the double $D(A)$ of a Hopf algebra $A$ in a braided autonomous category in such a way that the center of the category of $A$-modules is the braided category of $D(A)$-modules (generalizing the Drinfeld double). On the other hand, the canonical distributive law $\Omega$ also lifts $Z_{T}$ to a Hopf monad $\tilde{Z}_{T}^{\Omega}$ on $T-\mathcal{C}$, and $\tilde{Z}_{T}^{\Omega}\left(\mathbb{1}, T_{0}\right)$ is the coend of $T-\mathcal{C}$. For $T=Z$, this gives an explicit description of the Hopf algebra structure of the coend of $\mathcal{Z}(\mathcal{C})$ in terms of the structural morphisms of $\mathcal{C}$. Such a description is useful in quantum topology, especially when $\mathcal{C}$ is a spherical fusion category, as $\mathcal{Z}(\mathcal{C})$ is then modular.
\end{abstract}

\section{Contents}

Introduction 1226

1. Preliminaries and notation $\quad 1228$

2. Hopf monads and their modules 1233

3. Hopf monads, comonoidal adjunctions, and coends 1239

4. Distributive laws and liftings $\quad 1244$

5. The centralizer of a Hopf monad 1248

6. The double of a Hopf monad 1258

7. The centralizer of a Hopf monad on a category of modules 1261

8. The double of a Hopf algebra in a braided category 1267

9. Hopf monads and fusion categories $\quad 1275$

\begin{tabular}{ll} 
References & 1279 \\
\hline
\end{tabular}

Received by the editors June 5, 2009 and, in revised form, March 4, 2010.

2010 Mathematics Subject Classification. Primary 16W30, 18C20, 18 D10.

(C)2011 American Mathematical Society Reverts to public domain 28 years from publication 


\section{INTRODUCTION}

The center $\mathcal{Z}(\mathcal{C})$ of an autonomous category $\mathcal{C}$, introduced by Drinfeld, is a braided autonomous category. This construction establishes a bridge between the non-braided world and the braided world. It is useful, in particular, for comparing quantum invariants of 3 manifolds. Indeed, the center $\mathcal{Z}(\mathcal{C})$ of spherical fusion category $\mathcal{C}$ is modular (see Mü03], and it is conjectured that the Turaev-Viro invariant $\mathrm{TV}_{\mathcal{C}}$ (as revisited in [BW96]) is equal to the Reshetikhin-Turaev invariant $\mathrm{RT}_{\mathcal{Z}(\mathcal{C})}($ see RT91, Tur94]).

Let $\mathcal{C}$ be an autonomous category. If the coend

$$
Z(X)=\int^{Y \in \mathcal{C}}{ }^{\vee} Y \otimes X \otimes Y
$$

exists for all object $X$ of $\mathcal{C}$, then Day and Street DS07] showed that $Z$ is a monad on $\mathcal{C}$ and the center $\mathcal{Z}(\mathcal{C})$ is isomorphic to the category $Z$ - $\mathcal{C}$ of $Z$-modules in $\mathcal{C}$ (also called $Z$-algebras). By Tannaka reconstruction, we endow the $\operatorname{monad} Z$ with a quasitriangular Hopf monad structure which reflects the braided autonomous structure of $\mathcal{Z}(\mathcal{C})$ in the sense that $\mathcal{Z}(\mathcal{C}) \simeq Z$ - $\mathcal{C}$ as braided categories. The notion of a Hopf monad, which generalizes Hopf algebras to the non-braided (and nonlinear) setting, was introduced in [BV07] for this very purpose.

Given a modular category $\mathcal{B}$, the original construction of the Reshetikhin-Turaev invariant $\mathrm{RT}_{\mathcal{B}}$ involves the simple objects of $\mathcal{B}$ (see [RT91, Tur94]). However, when $\mathcal{B}=\mathcal{Z}(\mathcal{C})$ is the center of a spherical fusion category $\mathcal{C}$, we cannot compute explicitly by this method the invariant $\mathrm{RT}_{\mathcal{Z}(\mathcal{C})}$ in terms of $\mathcal{C}$, since there is no workable description of the simple objects of $\mathcal{Z}(\mathcal{C})$. Now Lyubashenko gave an alternate construction of the invariant $\mathrm{RT}_{\mathcal{B}}$ by means of the coend of the category $\mathcal{B}$, which is a Hopf algebra in $\mathcal{B}$ (see Lyu95, BV05]). So, to compute $\mathrm{RT}_{\mathcal{Z}(\mathcal{C})}$, all we need is an explicit description (in terms of $\mathcal{C}$ ) of the coend of $\mathcal{Z}(\mathcal{C})$ and its algebraic structure. To arrive at such a description, we develop the theory of the double of a Hopf monad.

Let $T$ be a Hopf monad on an autonomous category $\mathcal{C}$. We denote by $T$ - $\mathcal{C}$ the category of $T$-modules (also called $T$-algebras), which is autonomous. Assume $T$ is centralizable, meaning that the coend

$$
Z_{T}(X)=\int^{Y \in \mathcal{C}} \vee_{T}(Y) \otimes X \otimes Y
$$

exists for every object $X$ of $\mathcal{C}$. We endow $Z_{T}$ with a structure of a Hopf monad on $\mathcal{C}$ and call $Z_{T}$ the centralizer of $T$. In particular, the monad $Z$ defined above is the centralizer of the identity endofunctor $1_{\mathcal{C}}$ of the category $\mathcal{C}$.

Using adjunction and exactness properties of Hopf monads, we show that the identity endofunctor $1_{T-\mathcal{C}}$ of the category $T$ - $\mathcal{C}$ is centralizable and

$$
U_{T} Z_{1_{T-\mathcal{C}}}=Z_{T} U_{T}
$$

where $U_{T}: T-\mathcal{C} \rightarrow \mathcal{C}$ is the forgetful functor. This equality means that the Hopf monad $Z_{1_{T-\mathcal{C}}}$ is a lift to $T$ - $\mathcal{C}$ of the Hopf monad $Z_{T}$. Extending Beck's theory of distributive laws to Hopf monads, we show that such a lift is encoded by an invertible comonoidal distributive law $\Omega: T Z_{T} \rightarrow Z_{T} T$, called the canonical distributive law of $T$. The coend of $T-\mathcal{C}$ is therefore $\left(Z_{T}(\mathbb{1}), Z_{T}\left(T_{0}\right) \Omega_{\mathbb{1}}\right)$. When $T$ is quasitriangular, this coend has a structure of a Hopf algebra in the braided autonomous category $T$ - $\mathcal{C}$, which we elucidate in terms of $T$. In particular, for $T=Z$, we obtain 
an explicit description of the coend of $\mathcal{Z}(\mathcal{C})$. The case of fusion categories is treated in detail.

The canonical distributive law $\Omega$ also endows the composition of $Z_{T}$ and $T$ with a Hopf monad structure, denoted by $D_{T}=Z_{T} \circ_{\Omega} T$ and called the double of $T$. We prove that $D_{T}$ is quasitriangular and give a braided isomorphism:

$$
D_{T^{-}} \mathcal{C} \simeq \mathcal{Z}(T-\mathcal{C}) .
$$

This construction of the double of a centralizable Hopf monad on an autonomous category generalizes the Drinfeld double to a non-braided setting. As an illustration, we apply it to Hopf monads associated with Hopf algebras in braided categories. This leads to the double $D(A)$ of a Hopf algebra $A$ in a braided autonomous category $\mathcal{B}$ (which is different from the double considered in [Bes97] as it involves the coend of $\mathcal{B}$ ). More precisely, the endofunctor ? $\otimes A$ is a Hopf monad on $\mathcal{B}$. Assuming $\mathcal{B}$ admits a coend $C$, the Hopf monad ? $\otimes A$ is centralizable, and its centralizer is of the form ? $\otimes Z(A)$, where $Z(A)={ }^{\vee} A \otimes C$ is a Hopf algebra in $\mathcal{B}$. The canonical distributive law of ? $\otimes A$ is of the form $\operatorname{id}_{1_{\mathcal{B}}} \otimes \Omega$, where $\Omega: Z(A) \otimes A \rightarrow A \otimes Z(A)$ is a distributive law of Hopf algebras. Then

$$
D(A)=A \otimes_{\Omega} Z(A)=A \otimes{ }^{\vee} A \otimes C
$$

is a quasitriangular Hopf algebra in $\mathcal{B}$ such that

$$
\mathcal{Z}\left(\mathcal{B}_{A}\right) \simeq \mathcal{B}_{D(A)} \simeq{ }_{D(A)} \mathcal{B} \simeq \overline{\mathcal{Z}\left({ }_{A} \mathcal{B}\right)}
$$

as braided categories, where ${ }_{A} \mathcal{B}$ and $\mathcal{B}_{A}$ denote the categories of left and right $A$-modules in $\mathcal{B}$. In this context, a Hopf algebra $B$ in $\mathcal{B}$ is quasitriangular if it is endowed with an R-matrix, that is, a morphism $r: C \otimes C \rightarrow B \otimes B$ which encodes a braiding on $\mathcal{B}_{B}$ (or equivalently ${ }_{B} \mathcal{B}$ ). When $\mathcal{B}$ is the category of finite-dimensional vector spaces over a field $\mathbb{k}$, we recover the usual definition of an R-matrix and the Drinfeld double of a Hopf algebra $H$. Indeed, in that case, $C=\mathbb{k}, Z(H)=H^{* \text { cop }}$, and $D(H)=H \otimes_{\Omega} H^{* \text { cop }}$.

The canonical distributive law of a Hopf monad is in fact naturally defined in a more general setting. Let $T$ be a Hopf monad on an autonomous category $\mathcal{C}$ and $Q$ be a Hopf monad on $T$ - $\mathcal{C}$. Their cross product $Q \rtimes T=U_{T} Q F_{T}$ is a Hopf monad on $\mathcal{C}$, where $F_{T}: \mathcal{C} \rightarrow T$ - $\mathcal{C}$ is the left adjoint of the forgetful functor $U_{T}: T-\mathcal{C} \rightarrow \mathcal{C}$. If $Q \rtimes T$ is centralizable, then so is $Q$, and the Hopf monad $Z_{Q}$ is a lift to $T$ - $\mathcal{C}$ of the Hopf monad $Z_{Q \rtimes T}$ :

$$
U_{T} Z_{Q}=Z_{Q \rtimes T} U_{T}
$$

From this we obtain a canonical distributive law $\Omega: T Z_{Q \rtimes T} \rightarrow Z_{Q \rtimes T} T$ and a Hopf monad $D_{Q, T}=Z_{Q \rtimes T} \circ_{\Omega} T$ on $\mathcal{C}$. Moreover, we show

$$
D_{Q, T^{-}} \mathcal{C} \simeq \mathcal{Z}_{Q}(T-\mathcal{C})
$$

where $\mathcal{Z}_{Q}(T-\mathcal{C})$ is the center of $T$ - $\mathcal{C}$ relative to $Q$. When $Q=\mathrm{id}_{T-\mathcal{C}}$, we obtain the previous results.

This paper is organized as follows. In Section 1, we review several facts about monoidal categories, Hopf algebras in braided categories, and graphical calculus (which flows from bottom to top in this paper). Section 2 recalls the definition and elementary properties of Hopf monads. Section 3 deals with comonoidal adjunctions, exactness properties, and cross products of Hopf monads. In Section 4 we briefly recall the basic results of Beck's theory of distributive laws and extend them to the Hopf monad setting. In Section 5 , we define the centralizer $Z_{T}$ of a Hopf 
monad $T$ on $\mathcal{C}$ and relate it to the center $\mathcal{Z}_{T}(\mathcal{C})$ of $\mathcal{C}$ relative to $T$. In Section 6 , we define the canonical distributive law $\Omega$ of $T$ over $Z_{T}$ and the double $D_{T}=Z_{T} \circ_{\Omega} T$, and state their categorical properties. In Section 7 , we study the centralizer $Z_{Q}$ of a Hopf monad $Q$ on $T-\mathcal{C}$ and construct the canonical distributive law of $T$ over $Z_{Q \rtimes T}$. Section 8 is devoted to Hopf monads on a braided category $\mathcal{B}$. In particular, we define the double $D(A)$ of a Hopf algebra $A$ in a braided autonomous category. In Section 9, we treat the case of the center of a fusion category.

\section{Preliminaries and notation}

In this section, in order to fix notation and conventions, we review basic notions and facts concerning monoidal categories, (co)monoidal functors and natural transformations (see Mac98), autonomous categories, braided categories and functors, categorical centers (see [Kas95]), Hopf algebras in braided categories and their modules (see Maj95), and graphical calculus.

1.1. Categories. Unless otherwise specified, categories are small and monoidal categories are strict.

If $\mathcal{C}$ is a category, we denote by $\operatorname{Ob}(\mathcal{C})$ the set of objects of $\mathcal{C}$ and by $\operatorname{Hom}_{\mathcal{C}}(X, Y)$ the set of morphisms in $\mathcal{C}$ from an object $X$ to an object $Y$. The identity functor of $\mathcal{C}$ will be denoted by $1_{\mathcal{C}}$. We denote by $\mathcal{C}^{\text {op }}$ the opposite category (where the arrows are reversed).

Let $\mathcal{C}, \mathcal{D}$ be two categories. Functors from $\mathcal{C}$ to $\mathcal{D}$ are the objects of a category $\operatorname{Fun}(\mathcal{C}, \mathcal{D})$. Given two functors $F, G: \mathcal{C} \rightarrow \mathcal{D}$, a natural transformation $\alpha: F \rightarrow G$ is a family $\left\{\alpha_{X}: F(X) \rightarrow G(X)\right\}_{X \in \mathrm{Ob}(\mathcal{C})}$ of morphisms in $\mathcal{D}$ satisfying the following functoriality condition: $\alpha_{Y} F(f)=G(f) \alpha_{X}$ for every morphism $f: X \rightarrow Y$ in $\mathcal{C}$. We denote by $\operatorname{Hom}(F, G)$ the set $\operatorname{Hom}_{\mathrm{Fun}(\mathcal{C}, \mathcal{D})}(F, G)$ of natural transformations from $F$ to $G$ and by id ${ }_{F}$ the identity natural transformation of a functor $F$.

If $\mathcal{C}, \mathcal{C}^{\prime}$ are two categories, we denote by $\sigma_{\mathcal{C}, \mathcal{C}^{\prime}}$ the flip functor $\mathcal{C} \times \mathcal{C}^{\prime} \rightarrow \mathcal{C}^{\prime} \times \mathcal{C}$ defined by $\left(X, X^{\prime}\right) \mapsto\left(X^{\prime}, X\right)$.

1.2. Monoidal categories. Let $\mathcal{C}$ be a monoidal category with monoidal product $\otimes: \mathcal{C} \times \mathcal{C} \rightarrow \mathcal{C}$ and unit object $\mathbb{1}$. For $n \geq 0$, we denote by $\bigotimes_{n}$ the functor

$$
\otimes_{n}: \mathcal{C}^{n}=\underbrace{\mathcal{C} \times \cdots \times \mathcal{C}}_{n \text { times }} \rightarrow \mathcal{C}, \quad\left(X_{1}, \ldots, X_{n}\right) \mapsto X_{1} \otimes \cdots \otimes X_{n} .
$$

Note that $\otimes_{0}$ is the constant functor equal to $\mathbb{1}, \otimes_{1}=\mathrm{id}_{1_{\mathcal{C}}}$, and $\otimes_{2}=\otimes$.

For a family of functors $\left\{F_{i}: \mathcal{A}_{i} \rightarrow \mathcal{C}\right\}_{1 \leq i \leq n}$, set

$$
F_{1} \otimes \cdots \otimes F_{n}=\otimes_{n} \circ\left(F_{1}, \ldots, F_{n}\right): \mathcal{A}_{1} \times \cdots \times \mathcal{A}_{n} \rightarrow \mathcal{C} .
$$

For a functor $F: \mathcal{A} \rightarrow \mathcal{C}$, set

$$
F^{\otimes n}=\underbrace{F \otimes \cdots \otimes F}_{n \text { times }}: \mathcal{A}^{n} \rightarrow \mathcal{C} .
$$

If $\mathcal{C}$ is a monoidal category, we denote by $\mathcal{C}^{\otimes \mathrm{op}}$ the monoidal category $\left(\mathcal{C}, \otimes^{\mathrm{op}}, \mathbb{1}\right)$, where $X \otimes^{\text {op }}=Y \otimes X$, and by $\mathcal{C}^{\text {op }}$ the monoidal category $\left(\mathcal{C}^{\mathrm{op}}, \otimes, \mathbb{1}\right)$. 
1.3. Monoidal functors. Let $(\mathcal{C}, \otimes, \mathbb{1})$ and $(\mathcal{D}, \otimes, \mathbb{1})$ be two monoidal categories. A monoidal functor from $\mathcal{C}$ to $\mathcal{D}$ is a triple $\left(F, F_{2}, F_{0}\right)$, where $F: \mathcal{C} \rightarrow \mathcal{D}$ is a functor,

$$
F_{2}=\left\{F_{2}(X, Y): F(X) \otimes F(Y) \rightarrow F(X \otimes Y)\right\}_{X, Y \in \mathrm{Ob}(\mathcal{C})}
$$

is a natural transformation (from $F \otimes F$ to $F \otimes$ ), and $F_{0}: \mathbb{1} \rightarrow F(\mathbb{1}$ ) is a morphism in $\mathcal{D}$, such that

$$
\begin{aligned}
& F_{2}(X, Y \otimes Z)\left(\operatorname{id}_{F(X)} \otimes F_{2}(Y, Z)\right)=F_{2}(X \otimes Y, Z)\left(F_{2}(X, Y) \otimes \operatorname{id}_{F(Z)}\right), \\
& F_{2}(X, \mathbb{1})\left(\operatorname{id}_{F(X)} \otimes F_{0}\right)=\operatorname{id}_{F(X)}=F_{2}(\mathbb{1}, X)\left(F_{0} \otimes \operatorname{id}_{F(X)}\right),
\end{aligned}
$$

for all objects $X, Y, Z$ of $\mathcal{C}$.

A monoidal functor $\left(F, F_{2}, F_{0}\right)$ is said to be strong (resp. strict) if $F_{2}$ and $F_{0}$ are isomorphisms (resp. identities).

By a monoidal isomorphism, we mean a strong monoidal functor which is an isomorphism.

1.4. Monoidal natural transformations. Let $F: \mathcal{C} \rightarrow \mathcal{D}$ and $G: \mathcal{C} \rightarrow \mathcal{D}$ be two monoidal functors. A natural transformation $\varphi: F \rightarrow G$ is monoidal if it satisfies

$$
\varphi_{X \otimes Y} F_{2}(X, Y)=G_{2}(X, Y)\left(\varphi_{X} \otimes \varphi_{Y}\right) \quad \text { and } \quad G_{0}=\varphi_{\mathbb{1}} F_{0}
$$

1.5. Comonoidal functors. Let $(\mathcal{C}, \otimes, \mathbb{1})$ and $(\mathcal{D}, \otimes, \mathbb{1})$ be two monoidal categories. A comonoidal functor 1 from $\mathcal{C}$ to $\mathcal{D}$ is a triple $\left(F, F_{2}, F_{0}\right)$, where $F: \mathcal{C} \rightarrow \mathcal{D}$ is a functor,

$$
F_{2}=\left\{F_{2}(X, Y): F(X \otimes Y) \rightarrow F(X) \otimes F(Y)\right\}_{X, Y \in \mathrm{Ob}(\mathcal{C})}
$$

is a natural transformation (from $F \otimes$ to $F \otimes F$ ), and $F_{0}: F(\mathbb{1}) \rightarrow \mathbb{1}$ is a morphism in $\mathcal{D}$ such that

$$
\begin{aligned}
& \left(\operatorname{id}_{F(X)} \otimes F_{2}(Y, Z)\right) F_{2}(X, Y \otimes Z)=\left(F_{2}(X, Y) \otimes \operatorname{id}_{F(Z)}\right) F_{2}(X \otimes Y, Z), \\
& \left(\operatorname{id}_{F(X)} \otimes F_{0}\right) F_{2}(X, \mathbb{1})=\operatorname{id}_{F(X)}=\left(F_{0} \otimes \operatorname{id}_{F(X)}\right) F_{2}(\mathbb{1}, X),
\end{aligned}
$$

for all objects $X, Y, Z$ of $\mathcal{C}$.

We denote by $F_{3}: F \otimes_{3} \rightarrow F^{\otimes 3}$ the natural transformation defined by

$$
\begin{aligned}
F_{3}(X, Y, Z) & =\left(\operatorname{id}_{F(X)} \otimes F_{2}(Y, Z)\right) F_{2}(X, Y \otimes Z) \\
& =\left(F_{2}(X, Y) \otimes \operatorname{id}_{F(Z)}\right) F_{2}(X \otimes Y, Z) .
\end{aligned}
$$

A comonoidal functor $\left(F, F_{2}, F_{0}\right)$ is said to be strong (resp. strict) if $F_{2}$ and $F_{0}$ are isomorphisms (resp. identities). In that case, $\left(F, F_{2}^{-1}, F_{0}^{-1}\right)$ is a strong (resp. strict) monoidal functor.

1.6. Comonoidal natural transformations. Let $F: \mathcal{C} \rightarrow \mathcal{D}$ and $G: \mathcal{C} \rightarrow \mathcal{D}$ be two comonoidal functors. A natural transformation $\varphi: F \rightarrow G$ is comonoidal if it satisfies

$$
G_{2}(X, Y) \varphi_{X \otimes Y}=\left(\varphi_{X} \otimes \varphi_{Y}\right) F_{2}(X, Y) \quad \text { and } \quad G_{0} \varphi_{\mathbb{1}}=F_{0}
$$

\footnotetext{
${ }^{1}$ Comonoidal functors are also called opmonoidal functors.
} 
1.7. Autonomous categories. Recall that a duality in a monoidal category $\mathcal{C}$ is a quadruple $(X, Y, e, d)$, where $X, Y$ are objects of $\mathcal{C}, e: X \otimes Y \rightarrow \mathbb{1}$ (the evaluation) and $d: \mathbb{1} \rightarrow Y \otimes X$ (the coevaluation) are morphisms in $\mathcal{C}$ such that

$$
\left(e \otimes \operatorname{id}_{X}\right)\left(\operatorname{id}_{X} \otimes d\right)=\operatorname{id}_{X} \quad \text { and } \quad\left(\operatorname{id}_{Y} \otimes e\right)\left(d \otimes \operatorname{id}_{Y}\right)=\operatorname{id}_{Y} .
$$

Then $(X, e, d)$ is a left dual of $Y$ and $(Y, e, d)$ is a right dual of $X$.

If $D=(X, Y, e, d)$ and $D^{\prime}=\left(X^{\prime}, Y^{\prime}, e^{\prime}, d^{\prime}\right)$ are two dualities, two morphisms $f: X \rightarrow X^{\prime}$ and $g: Y^{\prime} \rightarrow Y$ are in duality with respect to $D$ and $D^{\prime}$ if

$$
\left.e^{\prime}\left(f \otimes \operatorname{id}_{Y^{\prime}}\right)=e\left(\operatorname{id}_{X} \otimes g\right) \quad \text { (or, equivalently, }\left(\operatorname{id}_{Y^{\prime}} \otimes f\right) d=\left(g \otimes \mathrm{id}_{X}\right) d^{\prime}\right) .
$$

In that case we write $f={ }^{\vee} g_{D, D^{\prime}}$ and $g=f_{D, D^{\prime}}^{\vee}$, or simply $f={ }^{\vee} g$ and $g=f^{\vee}$. Note that this defines a bijection between $\operatorname{Hom}_{\mathcal{C}}\left(X, X^{\prime}\right)$ and $\operatorname{Hom}_{\mathcal{C}}\left(Y^{\prime}, Y\right)$.

Left and right duals, if they exist, are essentially unique: if $(Y, e, d)$ and $\left(Y^{\prime}, e^{\prime}, d^{\prime}\right)$ are right duals of some object $X$, then there exists a unique isomorphism $u: Y \rightarrow Y^{\prime}$ such that $e^{\prime}=e\left(\operatorname{id}_{X} \otimes u^{-1}\right)$ and $d^{\prime}=\left(u \otimes \operatorname{id}_{X}\right) d$.

A left autonomous (resp. right autonomous, resp. autonomous) category is a monoidal category for which every object admits a left dual (resp. a right dual, resp. both a left and a right dual).

Assume $\mathcal{C}$ is a left autonomous category and, for each object $X$, pick a left dual $\left({ }^{\vee} X, \mathrm{ev}_{X}, \operatorname{coev}_{X}\right)$. This data defines a strong monoidal functor ${ }^{\vee} ?: \mathcal{C}^{\mathrm{op}, \otimes \mathrm{op}} \rightarrow \mathcal{C}$.

Likewise, if $\mathcal{C}$ is a right autonomous category, then picking a right dual $\left(X^{\vee}, \widetilde{\mathrm{ev}}_{X}\right.$, $\widetilde{\operatorname{coev}}_{X}$ ) for each object $X$ defines a strong monoidal functor $?^{\vee}: \mathcal{C}^{\mathrm{op}, \otimes \mathrm{op}} \rightarrow \mathcal{C}$.

Subsequently, when dealing with left or right autonomous categories, we shall always assume tacitly that left duals or right duals have been chosen. Moreover, in formulae, we abstain from writing the following canonical isomorphisms:

$$
\begin{array}{ll}
{ }^{\vee} ?_{2}(X, Y):{ }^{\vee} Y \otimes{ }^{\vee} X \rightarrow{ }^{\vee}(X \otimes Y), & { }^{\vee} ?_{0}: \mathbb{1} \rightarrow{ }^{\vee} \mathbb{1}, \\
?_{2}^{\vee}(X, Y): Y^{\vee} \otimes X^{\vee} \rightarrow(X \otimes Y)^{\vee}, & ?_{0}^{\vee}: \mathbb{1} \rightarrow \mathbb{1}^{\vee},
\end{array}
$$

and

$$
\begin{aligned}
& \left(\widetilde{\operatorname{ev}}_{X} \otimes \operatorname{idv}_{\left(X^{\vee}\right)}\right)\left(\operatorname{id}_{X} \otimes \operatorname{coev}_{X} \vee\right): X \rightarrow{ }^{\vee}\left(X^{\vee}\right), \\
& \left.\left(\operatorname{id}_{(\vee X}\right)^{\vee} \otimes \operatorname{ev}_{X}\right)\left(\widetilde{\operatorname{coev}^{\vee}}{ }_{X} \otimes \operatorname{id}_{X}\right): X \rightarrow\left({ }^{\vee} X\right)^{\vee} \text {. }
\end{aligned}
$$

1.8. Braided categories. Recall that a braiding on a monoidal category $\mathcal{C}$ is a natural isomorphism

$$
\tau=\left\{\tau_{X, Y}: X \otimes Y \rightarrow Y \otimes X\right\}_{X, Y \in \mathrm{Ob}(\mathcal{C})}
$$

$\left(\right.$ from $\otimes$ to $\left.\otimes \sigma_{\mathcal{C}, \mathcal{C}}\right)$ such that

$$
\tau_{X, Y \otimes Z}=\left(\operatorname{id}_{Y} \otimes \tau_{X, Z}\right)\left(\tau_{X, Y} \otimes \operatorname{id}_{Z}\right) \quad \text { and } \quad \tau_{X \otimes Y, Z}=\left(\tau_{X, Z} \otimes \operatorname{id}_{Y}\right)\left(\operatorname{id}_{X} \otimes \tau_{Y, Z}\right) .
$$

A braided category is a monoidal category endowed with a braiding.

The mirror of a braiding $\tau$ is the braiding $\bar{\tau}$ defined by $\bar{\tau}_{X, Y}=\tau_{Y, X}^{-1}$. The mirror of a braided category $\mathcal{B}$ is the braided category $\overline{\mathcal{B}}$ which coincides with $\mathcal{B}$ as a monoidal category but is endowed with the mirror braiding. 
If $\mathcal{B}$ is braided with braiding $\tau$, then $\mathcal{B}^{\otimes \circ \mathrm{op}}$ is braided with braiding $\tau^{\mathrm{o}}$ defined by $\tau_{X, Y}^{\mathrm{o}}=\tau_{Y, X}$. Note that $\tau \mapsto \tau^{\mathrm{O}}$ is a bijection between braidings on $\mathcal{B}$ and braidings on $\mathcal{B}^{\otimes \text { op }}$.

1.9. Braided functors. A braided functor between two braided categories $\mathcal{B}$ and $\mathcal{B}^{\prime}$ is a strong monoidal functor $F: \mathcal{B} \rightarrow \mathcal{B}^{\prime}$ such that

$$
F\left(\tau_{X, Y}\right) F_{2}(X, Y)=F_{2}(Y, X) \tau_{F(X), F(Y)}^{\prime}
$$

for all objects $X, Y$ of $\mathcal{B}$, where $\tau$ and $\tau^{\prime}$ are the braidings of $\mathcal{B}$ and $\mathcal{B}^{\prime}$.

Example 1.1. If $\mathcal{B}$ is a braided category with braiding $\tau$, the monoidal functor $\left(1_{\mathcal{B}}, \tau, \mathrm{id}_{\mathbb{1}}\right): \mathcal{B}^{\otimes \mathrm{op}} \rightarrow \mathcal{B}$ is a braided isomorphism.

1.10. The center of a monoidal category. Let $\mathcal{C}$ be a monoidal category. A left half braiding of $\mathcal{C}$ is a pair $(M, \sigma)$, where $M$ is an object of $\mathcal{C}$ and

$$
\sigma=\left\{\sigma_{X}: M \otimes X \rightarrow X \otimes M\right\}_{X \in \mathrm{Ob}(\mathcal{C})}
$$

is a natural transformation (from $M \otimes 1_{\mathcal{C}}$ to $1_{\mathcal{C}} \otimes M$ ) such that:

(i) $\sigma_{Y \otimes Z}=\left(\operatorname{id}_{Y} \otimes \sigma_{Z}\right)\left(\sigma_{Y} \otimes \mathrm{id}_{Z}\right)$ for all $Y, Z \in \mathrm{Ob}(\mathcal{C})$;

(ii) $\sigma_{\mathbb{1}}=\mathrm{id}_{M}$;

(iii) $\sigma$ is an isomorphism.

Note that if $\mathcal{C}$ is autonomous, (iii) is a consequence of (i) and (ii).

The center of $\mathcal{C}$ is the braided category $\mathcal{Z}(\mathcal{C})$ defined as follows. Its objects are left half braidings of $\mathcal{C}$. A morphism in $\mathcal{Z}(\mathcal{C})$ from $(M, \sigma)$ to $\left(M^{\prime}, \sigma^{\prime}\right)$ is a morphism $f: M \rightarrow M^{\prime}$ in $\mathcal{C}$ such that $\left(\operatorname{id}_{1_{\mathcal{C}}} \otimes f\right) \sigma=\sigma^{\prime}\left(f \otimes \operatorname{id}_{1_{\mathcal{C}}}\right)$. The monoidal product and braiding $\tau$ are

$$
(M, \sigma) \otimes(N, \gamma)=\left(M \otimes N,\left(\sigma \otimes \operatorname{id}_{N}\right)\left(\operatorname{id}_{M} \otimes \gamma\right)\right) \quad \text { and } \quad \tau_{(M, \sigma),(N, \gamma)}=\sigma_{N} .
$$

Note that if $\mathcal{C}$ is autonomous, so is $\mathcal{Z}(\mathcal{C})$.

Remark 1.2. Likewise, define a right half braiding of a monoidal category $\mathcal{C}$ to be a pair $(M, \sigma)$, where $M$ is an object of $\mathcal{C}$ and $\sigma: 1_{\mathcal{C}} \otimes M \rightarrow M \otimes 1_{\mathcal{C}}$ is a natural transformation satisfying analogous axioms. Right half braidings form a braided category $\mathcal{Z}^{\prime}(\mathcal{C})$ with braiding: $\tau_{(M, \sigma),(N, \gamma)}^{\prime}=\gamma_{M}$. We have

$$
\mathcal{Z}^{\prime}(\mathcal{C})=\mathcal{Z}\left(\mathcal{C}^{\text {op }}\right)^{\text {op }}=\mathcal{Z}\left(\mathcal{C}^{\otimes \text { op }}\right)^{\otimes \text { op }}
$$

The braided category $\mathcal{Z}^{\prime}(\mathcal{C})$ is isomorphic to the mirror of $\mathcal{Z}(\mathcal{C})$ via the braided isomorphism given by $(M, \sigma) \mapsto\left(M, \sigma^{-1}\right)$.

1.11. Algebras, bialgebras, and Hopf algebras in categories. Let $\mathcal{C}$ be a monoidal category. An algebra in $\mathcal{C}$ is an object $A$ of $\mathcal{C}$ endowed with morphisms $m: A \otimes A \rightarrow A$ (the product) and $u: \mathbb{1} \rightarrow A$ (the unit) such that

$$
m\left(m \otimes \mathrm{id}_{A}\right)=m\left(\operatorname{id}_{A} \otimes m\right) \quad \text { and } \quad m\left(\operatorname{id}_{A} \otimes u\right)=\operatorname{id}_{A}=m\left(u \otimes \operatorname{id}_{A}\right) .
$$

A coalgebra in $\mathcal{C}$ is an object $C$ of $\mathcal{C}$ endowed with morphisms $\Delta: C \rightarrow C \otimes C$ (the coproduct) and $\varepsilon: C \rightarrow \mathbb{1}$ (the counit) such that

$$
\left(\Delta \otimes \operatorname{id}_{C}\right) \Delta=\left(\operatorname{id}_{C} \otimes \Delta\right) \Delta \quad \text { and } \quad\left(\operatorname{id}_{C} \otimes \varepsilon\right) \Delta=\operatorname{id}_{C}=\left(\varepsilon \otimes \operatorname{id}_{C}\right) \Delta .
$$


Let $\mathcal{B}$ be a braided category with braiding $\tau$. A bialgebra in $\mathcal{B}$ is an object $A$ of $\mathcal{B}$ endowed with an algebra structure $(m, u)$ and a coalgebra structure $(\Delta, \varepsilon)$ in $\mathcal{B}$ satisfying

$$
\begin{aligned}
\Delta m & =(m \otimes m)\left(\mathrm{id}_{A} \otimes \tau_{A, A} \otimes \mathrm{id}_{A}\right)(\Delta \otimes \Delta), & \Delta u & =u \otimes u, \\
\varepsilon m & =\varepsilon \otimes \varepsilon, & \varepsilon u & =\mathrm{id}_{\mathbb{1}} .
\end{aligned}
$$

Let $A$ be a bialgebra in $\mathcal{B}$. Set

$$
m^{\mathrm{op}}=m \tau_{A, A}^{-1} \quad \text { and } \quad \Delta^{\mathrm{cop}}=\tau_{A, A}^{-1} \Delta .
$$

Then $\left(A, m^{\mathrm{op}}, u, \Delta, \varepsilon\right)$ is a bialgebra in the mirror $\overline{\mathcal{B}}$ of $\mathcal{B}$, called the opposite of $A$, and denoted by $A^{\mathrm{op}}$. Similarly $\left(A, m, u, \Delta^{\mathrm{cop}}, \varepsilon\right)$ is a bialgebra in $\overline{\mathcal{B}}$, called the $c o-$ opposite of $A$, and denoted by $A^{\mathrm{cop}}$. Consequently $A^{\mathrm{cop}, \mathrm{op}}=\left(A^{\mathrm{cop}}\right)^{\mathrm{op}}$ is a bialgebra in $\mathcal{B}$ (with product $m \tau_{A, A}$ and coproduct $\tau_{A, A}^{-1} \Delta$ ).

An antipode for a bialgebra $A$ is a morphism $S: A \rightarrow A$ in $\mathcal{B}$ such that

$$
m\left(S \otimes \operatorname{id}_{A}\right) \Delta=u \varepsilon=m\left(\operatorname{id}_{A} \otimes S\right) \Delta .
$$

If it exists, an antipode is unique, and it is a morphism of bialgebras $A \rightarrow A^{\text {cop,op }}$. A Hopf algebra in $\mathcal{B}$ is a bialgebra in $\mathcal{B}$ which admits an invertible antipode.

If $A$ is a Hopf algebra in $\mathcal{B}$, with antipode $S$, then $A^{\text {op }}$ and $A^{\text {cop }}$ are Hopf algebras in the mirror $\overline{\mathcal{B}}$ of $\mathcal{B}$, with antipode $S^{-1}$.

1.12. Modules in categories. Let $(A, m, u)$ be an algebra in a monoidal category $\mathcal{C}$. A left $A$-module (in $\mathcal{C}$ ) is a pair $(M, r)$, where $M$ is an object of $\mathcal{C}$ and $r: A \otimes M \rightarrow M$ is a morphism in $\mathcal{C}$, such that

$$
r\left(m \otimes \mathrm{id}_{M}\right)=r\left(\mathrm{id}_{A} \otimes r\right) \quad \text { and } \quad r\left(u \otimes \operatorname{id}_{M}\right)=\operatorname{id}_{M} .
$$

An $A$ - linear morphism between two left $A$-modules $(M, r)$ and $(N, s)$ is a morphism $f: M \rightarrow N$ such that $f r=s\left(\operatorname{id}_{A} \otimes f\right)$. Hence the category ${ }_{A} \mathcal{C}$ of left $A$-modules. Likewise, one defines the category $\mathcal{C}_{A}$ of right $A$-modules.

Let $A$ be a bialgebra in a braided category $\mathcal{B}$. Then the category ${ }_{A} \mathcal{B}$ is monoidal, with unit object $(\mathbb{1}, \varepsilon)$ and monoidal product

$$
(M, r) \otimes(N, s)=(r \otimes s)\left(\operatorname{id}_{A} \otimes \tau_{A, M} \otimes \operatorname{id}_{N}\right)\left(\Delta \otimes \operatorname{id}_{M \otimes N}\right),
$$

where $\Delta$ and $\epsilon$ are the coproduct and counit of $A$, and $\tau$ is the braiding of $\mathcal{B}$. Likewise the category $\mathcal{B}_{A}$ is monoidal, with unit object $(\mathbb{1}, \varepsilon)$ and monoidal product

$$
(M, r) \otimes(N, s)=(r \otimes s)\left(\operatorname{id}_{M} \otimes \tau_{N, A} \otimes \operatorname{id}_{A}\right)\left(\Delta \otimes \operatorname{id}_{M \otimes N}\right) .
$$

Assume $\mathcal{B}$ is autonomous. Then ${ }_{A} \mathcal{B}$ is autonomous if and only if $\mathcal{B}_{A}$ is autonomous, if and only if $A$ is a Hopf algebra. If $A$ is a Hopf algebra, with antipode $S$, then the duals of a left $A$-module $(M, r)$ are

$$
\begin{aligned}
& { }^{\vee}(M, r)=\left({ }^{\vee} M,\left(\operatorname{ev}_{M} \otimes \operatorname{id}_{M}\right)\left(\operatorname{id}_{\vee_{M}} \otimes r\left(S \otimes \operatorname{id}_{M}\right) \otimes \operatorname{id}_{\vee_{M}}\right)\left(\tau_{A, \vee_{M}} \otimes \operatorname{coev}_{M}\right)\right), \\
& (M, r)^{\vee}=\left(M^{\vee},\left(\operatorname{id}_{M^{\vee}} \otimes \widetilde{\operatorname{ev}}_{M}\right)\left(\operatorname{id}_{M^{\vee}} \otimes r \tau_{A, M}^{-1} \otimes \operatorname{id}_{M^{\vee}}\right)\left(\widetilde{\operatorname{coev}}_{M} \otimes S^{-1} \otimes \operatorname{id}_{M^{\vee}}\right)\right),
\end{aligned}
$$

and the duals of a right $A$-module $(M, r)$ are

$$
\begin{aligned}
& { }^{\vee}(M, r)=\left({ }^{\vee} M,\left(\operatorname{ev}_{M} \otimes \operatorname{id}_{\vee_{M}}\right)\left(\operatorname{id}_{\vee_{M}} \otimes r \tau_{M, A}^{-1} \otimes \operatorname{id}_{\vee_{M}}\right)\left(\operatorname{id}_{\vee_{M}} \otimes S^{-1} \otimes \operatorname{coev}_{M}\right)\right), \\
& (M, r)^{\vee}=\left(M^{\vee},\left(\operatorname{id}_{M} \vee \otimes \widetilde{\operatorname{ev}}_{M}\right)\left(\operatorname{id}_{M^{\vee}} \otimes r\left(\operatorname{id}_{M} \otimes S\right) \otimes \operatorname{id}_{M^{\vee}}\right)\left(\widetilde{\operatorname{coev}_{M}} \otimes \tau_{M^{\vee}, A}\right)\right) .
\end{aligned}
$$


Remark 1.3. Let $A$ be a Hopf algebra in a braided category $\mathcal{B}$, with braiding $\tau$. The functor $F_{A}:{ }_{A} \mathcal{B} \rightarrow \mathcal{B}_{A}$, defined by $F_{A}(M, r)=\left(M, r \tau_{M, A}\left(\operatorname{id}_{M} \otimes S\right)\right)$ and $F_{A}(f)=f$, gives rise to a monoidal isomorphism of categories:

$$
F_{A}=\left(F_{A}, \tau, \mathbb{1}\right):\left({ }_{A} \mathcal{B}\right)^{\otimes \mathrm{op}} \rightarrow \mathcal{B}_{A} .
$$

Therefore braidings on ${ }_{A} \mathcal{B}$ are in bijection with braidings on $\mathcal{B}_{A}$. More precisely, if $c$ is a braiding on $\mathcal{B}_{A}$, then

$$
c_{(M, r),(N, s)}^{\prime}=\tau_{M, N} c_{F_{A}(N, s), F_{A}(M, r)} \tau_{N, M}^{-1}
$$

is a braiding on ${ }_{A} \mathcal{B}$, making $F_{A}$ braided, and the correspondence $c \mapsto c^{\prime}$ is bijective.

1.13. Penrose graphical calculus. We represent morphisms in a category by diagrams to be read from bottom to top. Thus we draw the identity id $X$ of an object $X$, a morphism $f: X \rightarrow Y$, and its composition with a morphism $g: Y \rightarrow Z$ as follows:

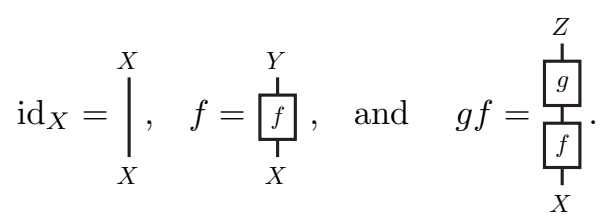

In a monoidal category, we represent the monoidal product of two morphisms $f: X \rightarrow Y$ and $g: U \rightarrow V$ by juxtaposition:

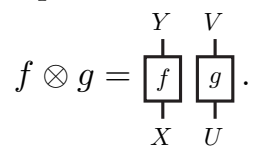

The duality morphisms of an autonomous category are depicted as

$$
\mathrm{ev}_{X}=\bigcap_{\vee_{X}}, \quad \operatorname{coev}_{X}=\bigcup^{X} \vee_{X}, \quad \widetilde{\mathrm{ev}}_{X}=\bigcap_{X}, \quad \text { and } \quad \widetilde{\operatorname{coev}}_{X}=\bigcup^{X^{\vee} X}
$$

The braiding $\tau$ of a braided category, and its inverse, are depicted as

$$
\tau_{X, Y}=Y_{X}^{Y} Y_{Y}^{X} \text { and } \tau_{Y, X}^{-1}=\searrow_{X}^{Y}
$$

Given a Hopf algebra $A$ in a braided category, we depict its product $m$, unit $u$, coproduct $\Delta$, counit $\varepsilon$, antipode $S$, and $S^{-1}$ as follows:

$$
m=\bigcap_{A}^{A}, \quad u=\left.\right|_{A} ^{A}, \quad \Delta=\bigcup_{A}^{A}, \quad \varepsilon=\uparrow_{A}^{A}, \quad S=\oint_{A}^{A}, \quad S^{-1}=\oint_{A}^{+} .
$$

\section{Hopf MONADS AND THEIR MODULES}

In this section, we review the notion of a Hopf monad. For a general treatment, we refer to BV07.

2.1. Monads. Let $\mathcal{C}$ be a category. Recall that the category $\operatorname{End}(\mathcal{C})$ of endofunctors of $\mathcal{C}$ is strict monoidal with composition for monoidal product and identity functor $1_{\mathcal{C}}$ for a unit object. A monad on $\mathcal{C}$ is an algebra in $\operatorname{End}(\mathcal{C})$, that is, a triple $(T, \mu, \eta)$, where $T: \mathcal{C} \rightarrow \mathcal{C}$ is a functor, $\mu: T^{2} \rightarrow T$ and $\eta: 1_{\mathcal{C}} \rightarrow T$ are natural transformations, such that

$$
\mu_{X} T\left(\mu_{X}\right)=\mu_{X} \mu_{T(X)} \quad \text { and } \quad \mu_{X} \eta_{T(X)}=\operatorname{id}_{T(X)}=\mu_{X} T\left(\eta_{X}\right)
$$

for any object $X$ of $\mathcal{C}$. 
Example 2.1. Let $A$ be an algebra in a monoidal category $\mathcal{C}$, with product $m$ and unit $u$. Then the endofunctor ? $\otimes A$ of $\mathcal{C}$, defined by $X \mapsto X \otimes A$, has a structure of a monad on $\mathcal{C}$ with product $\mu=\mathrm{id}_{1_{\mathcal{C}}} \otimes m$ and unit $\eta=\mathrm{id}_{1_{\mathcal{C}}} \otimes u$. Similarly, the endofunctor $A \otimes$ ? is a monad on $\mathcal{C}$ with product $m \otimes \operatorname{id}_{1_{\mathcal{C}}}$ and unit $u \otimes \operatorname{id}_{1_{\mathcal{C}}}$.

2.2. Bimonads. A bimonad 2 on a monoidal category $\mathcal{C}$ is a $\operatorname{monad}(T, \mu, \eta)$ on $\mathcal{C}$ such that the functor $T: \mathcal{C} \rightarrow \mathcal{C}$ is comonoidal and the natural transformations $\mu: T^{2} \rightarrow T$ and $\eta: 1_{\mathcal{C}} \rightarrow T$ are comonoidal. In other words, $T$ is endowed with a natural transformation $T_{2}: T \otimes \rightarrow T \otimes T$ and a morphism $T_{0}: T(\mathbb{1}) \rightarrow \mathbb{1}$ in $\mathcal{C}$ such that

$$
\begin{aligned}
& \left(\operatorname{id}_{T(X)} \otimes T_{2}(Y, Z)\right) T_{2}(X, Y \otimes Z)=\left(T_{2}(X, Y) \otimes \operatorname{id}_{T(Z)}\right) T_{2}(X \otimes Y, Z), \\
& \left(\operatorname{id}_{T(X)} \otimes T_{0}\right) T_{2}(X, \mathbb{1})=\operatorname{id}_{T(X)}=\left(T_{0} \otimes \operatorname{id}_{T(X)}\right) T_{2}(\mathbb{1}, X)
\end{aligned}
$$

and

$$
\begin{aligned}
& T_{2}(X, Y) \mu_{X \otimes Y}=\left(\mu_{X} \otimes \mu_{Y}\right) T_{2}(T(X), T(Y)) T\left(T_{2}(X, Y)\right), \\
& T_{0} \mu_{\mathbb{1}}=T_{0} T\left(T_{0}\right), \quad T_{2}(X, Y) \eta_{X \otimes Y}=\left(\eta_{X} \otimes \eta_{Y}\right), \quad T_{0} \eta_{\mathbb{1}}=\mathrm{id}_{\mathbb{1}} .
\end{aligned}
$$

Remark 2.2. A bimonad on a monoidal category $\mathcal{C}$ is nothing but an algebra in the strict monoidal category of comonoidal endofunctors of $\mathcal{C}$ (with a monoidal product $\circ$ and a unit object $1_{\mathcal{C}}$ ).

Remark 2.3. A bimonad $T$ on a monoidal category $\mathcal{C}=(\mathcal{C}, \otimes, \mathbb{1})$ may be viewed as a bimonad $T^{\mathrm{cop}}$ on the monoidal category $\mathcal{C}^{\otimes \mathrm{op}}=\left(\mathcal{C}, \otimes^{\mathrm{op}}, \mathbb{1}\right)$, with comonoidal structure $T_{2}^{\mathrm{cop}}(X, Y)=T_{2}(Y, X)$ and $T_{0}^{\mathrm{cop}}=T_{0}$. The bimonad $T^{\mathrm{cop}}$ is called the

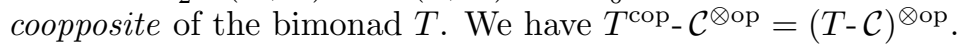

2.3. Antipodes. Right and left antipodes of a Hopf monad generalize the antipode of a Hopf algebra and its inverse. Let $(T, \mu, \eta)$ be a bimonad on a monoidal category $\mathcal{C}$.

Assume $\mathcal{C}$ is left autonomous. A left antipode for $T$ is a natural transformation $s^{l}=\left\{s_{X}^{l}: T\left({ }^{\vee} T(X)\right) \rightarrow{ }^{\vee} X\right\}_{X \in \mathrm{Ob}(\mathcal{C})}$ satisfying

$$
\begin{aligned}
& T_{0} T\left(\operatorname{ev}_{X}\right) T\left({ }^{\vee} \eta_{X} \otimes \operatorname{id}_{X}\right)=\operatorname{ev}_{T(X)}\left(s_{T(X)}^{l} T\left({ }^{\vee} \mu_{X}\right) \otimes \operatorname{id}_{T(X)}\right) T_{2}\left({ }^{\vee} T(X), X\right), \\
& \left(\eta_{X} \otimes \operatorname{id}_{\vee_{X}}\right) \operatorname{coev}_{X} T_{0}=\left(\mu_{X} \otimes s_{X}^{l}\right) T_{2}\left(T(X),{ }^{\vee} T(X)\right) T\left(\operatorname{coev}_{T(X)}\right),
\end{aligned}
$$

for every object $X$ of $\mathcal{C}$. By [BV07, Theorem 3.7], a left antipode $s^{l}$ is 'anti(co)multiplicative': for all objects $X, Y$ of $\mathcal{C}$,

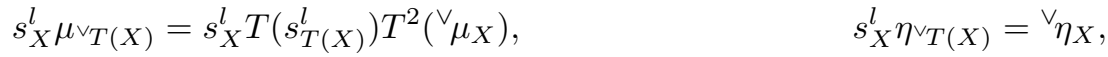

$$
\begin{aligned}
& s_{X \otimes Y}^{l} T\left({ }^{\vee} T_{2}(X, Y)\right)=\left(s_{Y}^{l} \otimes s_{X}^{l}\right) T_{2}\left({ }^{\vee} T(Y),{ }^{\vee} T(X)\right), \quad s_{\mathbb{1}}^{l} T\left({ }^{\vee} T_{0}\right)=T_{0} .
\end{aligned}
$$

Assume $\mathcal{C}$ is right autonomous. A right antipode for $T$ is a natural transformation $s^{r}=\left\{s_{X}^{r}: T\left(T(X)^{\vee}\right) \rightarrow X^{\vee}\right\}_{X \in \mathrm{Ob}(\mathcal{C})}$ satisfying

$$
\begin{aligned}
& T_{0} T\left(\widetilde{\operatorname{ev}}_{X}\right) T\left(\operatorname{id}_{X} \otimes \eta_{X}^{\vee}\right)=\widetilde{\operatorname{ev}}_{T(X)}\left(\operatorname{id}_{T(X)} \otimes s_{T(X)}^{r} T\left(\mu_{X}^{\vee}\right)\right) T_{2}\left(X, T(X)^{\vee}\right), \\
& \left(\operatorname{id}_{X} \vee \otimes \eta_{X}\right) \widetilde{\operatorname{cov}}_{X} T_{0}=\left(s_{X}^{r} \otimes \mu_{X}\right) T_{2}\left(T(X)^{\vee}, T(X)\right) T\left(\widetilde{\operatorname{cov}}_{T(X)}\right),
\end{aligned}
$$

\footnotetext{
${ }^{2}$ Bimonads were introduced in Moe02] under the name 'Hopf monads', which we prefer to reserve for bimonads with antipodes by analogy with Hopf algebras.
} 
for every object $X$ of $\mathcal{C}$. By [BV07, Theorem 3.7], a right antipode $s^{r}$ is also 'anti-(co)multiplicative': for all objects $X, Y$ of $\mathcal{C}$,

$$
\begin{array}{ll}
s_{X}^{r} \mu_{T(X)^{\vee}}=s_{X}^{r} T\left(s_{T(X)}^{r}\right) T^{2}\left(\mu_{X}^{\vee}\right), & s_{X}^{r} \eta_{T(X)^{\vee}}=\eta_{X} \vee, \\
s_{X \otimes Y}^{r} T\left(T_{2}(X, Y)^{\vee}\right)=\left(s_{Y}^{r} \otimes s_{X}^{r}\right) T_{2}\left(T(Y)^{\vee}, T(X)^{\vee}\right), & s_{\mathbb{1}}^{r} T\left(T_{0}^{\vee}\right)=T_{0} .
\end{array}
$$

Note that if a left (resp. right) antipode exists, then it is unique. Furthermore, when both exist, the left antipode $s^{l}$ and the right antipode $s^{r}$ are 'inverse' to each other in the sense that $\operatorname{id}_{T(X)}=s_{\vee_{T(X)}^{r}}^{r} T\left(\left(s_{X}^{l}\right)^{\vee}\right)=s_{T(X) \vee}^{l} T\left({ }^{\vee}\left(s_{X}^{r}\right)\right)$ for any object $X$ of $\mathcal{C}$.

2.4. Hopf monads. A Hopf monad is a bimonad on an autonomous category which has a left antipode and a right antipode.

Hopf monads generalize Hopf algebras in a non-braided setting. In particular, finite-dimensional Hopf algebras and several generalizations (Hopf algebras in braided autonomous categories, bialgebroids, etc.) provide examples of Hopf monads. In fact, any comonoidal adjunction between autonomous categories gives rise to a Hopf monad (see Theorem 3.3). It turns out that much of the theory of finite-dimensional Hopf algebras (such as the decomposition of Hopf modules, the existence of integrals, Maschke's criterium of semisimplicity, etc.) extends to Hopf monads; see BV07.

Example 2.4 (Hopf monads associated with Hopf algebras). Let $A$ be a Hopf algebra in a braided autonomous category $\mathcal{B}$, with braiding $\tau$. According to [BV07, the endofunctor ? $\otimes A$ of $\mathcal{B}$ has a structure of a Hopf monad on $\mathcal{B}$, with product $\mu=\mathrm{id}_{1_{\mathcal{B}}} \otimes m$, unit $\eta=\mathrm{id}_{1_{\mathcal{B}}} \otimes u$, comonoidal structure given by

$$
(? \otimes A)_{2}(X, Y)=\left(\operatorname{id}_{X} \otimes \tau_{Y, A} \otimes \operatorname{id}_{A}\right)\left(\mathrm{id}_{X \otimes Y} \otimes \Delta\right) \quad \text { and } \quad(? \otimes A)_{0}=\varepsilon,
$$

and left and right antipodes

$$
\begin{aligned}
& s_{X}^{l}=\left(\mathrm{ev}_{A} \otimes \mathrm{id}_{\vee_{X}}\right)\left(\mathrm{id}_{v_{A}} \otimes \tau v_{X, A}\right)\left(\mathrm{id}_{v_{A} \otimes \vee_{X}} \otimes S^{-1}\right), \\
& s_{X}^{r}=\left(\widetilde{\mathrm{ev}}_{A} \otimes \mathrm{id}_{X} \vee \tau_{A^{\vee} \otimes X^{\vee}, A}\left(\operatorname{id}_{A \vee} \otimes X^{\vee} \otimes S\right) .\right.
\end{aligned}
$$

Pictorially, the structural morphisms of $? \otimes A$ are

$$
\begin{aligned}
& \mu_{X}=\left.\right|_{X} ^{X} \bigcap_{A}^{A}, \quad \eta_{X}=\left.\right|_{X} ^{X} d^{A}, \quad(? \otimes A)_{2}(X, Y)=\left.\left.\right|_{X} ^{X}\right|_{Y} ^{A} Y_{A}^{Y},
\end{aligned}
$$

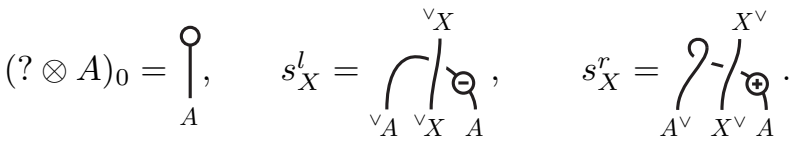

Similarly, the endofunctor $A \otimes$ ? of $\mathcal{B}$ has a structure of a Hopf monad on $\mathcal{B}$, with product $\mu=m \otimes \mathrm{id}_{1_{\mathcal{B}}}$, unit $\eta=u \otimes \mathrm{id}_{1_{\mathcal{B}}}$, comonoidal structure

$$
(A \otimes ?)_{2}(X, Y)=\left(\operatorname{id}_{A} \otimes \tau_{A, X} \otimes \operatorname{id}_{Y}\right)\left(\Delta \otimes \mathrm{id}_{X \otimes Y}\right) \quad \text { and } \quad(A \otimes ?)_{0}=\varepsilon,
$$

and left and right antipodes

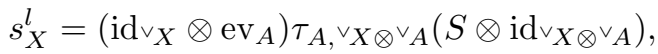

$$
\begin{aligned}
& s_{X}^{r}=\left(\operatorname{id}_{X} \vee \otimes \widetilde{e v}_{A}\right)\left(\tau_{A, X} \vee \otimes \operatorname{idv}_{A}\right)\left(S^{-1} \otimes \operatorname{id}_{X} \vee \otimes A^{\vee}\right) \text {. }
\end{aligned}
$$


Pictorially, the structural morphisms of $A \otimes ?$ are

$$
\begin{aligned}
& \mu_{X}=\left.\bigcap_{A}^{A}\right|_{A} ^{X}, \quad \eta_{X}=\left.b_{X}^{A}\right|_{X} ^{X}, \quad(A \otimes ?)_{2}(X, Y)=\left.\left.\left.\bigoplus_{A}^{A}\right|_{X} ^{X}\right|_{Y} ^{A}\right|_{Y} ^{Y},
\end{aligned}
$$

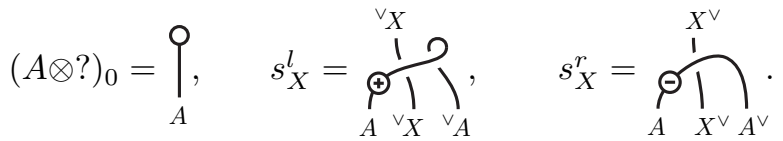

Example 2.5. The previous example can be extended to the non-braided setting as follows. Let $\mathcal{C}$ be an autonomous category and $(A, \sigma)$ be a Hopf algebra in the center $\mathcal{Z}(\mathcal{C})$ of $\mathcal{C}$ (see Section 1.10). Denote by $m, u, \Delta, \varepsilon, S$ the product, unit, coproduct, counit, and antipode of $(A, \sigma)$. Observe that $(A, m, u)$ is an algebra in $\mathcal{C}$. Then the endofunctor $A \otimes$ ? of $\mathcal{C}$ has a structure of a Hopf monad on $\mathcal{C}$, denoted by $A \otimes_{\sigma}$ ?, with product $\mu=m \otimes \mathrm{id}_{1_{\mathcal{C}}}$, unit $\eta=u \otimes \mathrm{id}_{1_{\mathcal{C}}}$, comonoidal structure

$$
\left(A \otimes_{\sigma} ?\right)_{2}(X, Y)=\left(\operatorname{id}_{A} \otimes \sigma_{X} \otimes \operatorname{id}_{Y}\right)\left(\Delta \otimes \operatorname{id}_{X \otimes Y}\right) \quad \text { and } \quad\left(A \otimes_{\sigma} ?\right)_{0}=\varepsilon,
$$

and left and right antipodes

$$
\begin{aligned}
& s_{X}^{l}=\left(\operatorname{id}_{X} \otimes \operatorname{ev}_{A}\right) \sigma \vee_{X} \otimes \vee_{A}\left(S \otimes \operatorname{id} \vee_{X} \otimes \vee_{A}\right), \\
& s_{X}^{r}=\left(\operatorname{id}_{X \vee} \otimes \tilde{e v}_{A}\right)\left(\sigma_{X} \vee \otimes \operatorname{id}_{A}\right)\left(S^{-1} \otimes \operatorname{id}_{X \vee} \otimes A^{\vee}\right) .
\end{aligned}
$$

Likewise, if $(A, \sigma)$ is a Hopf algebra in $\mathcal{Z}^{\prime}(\mathcal{C})$ (see Remark 1.2), then the endofunctor $? \otimes A$ of $\mathcal{C}$ has a structure of a Hopf monad on $\mathcal{C}$, denoted by ? $\otimes_{\sigma} A$, with product $\mu=\mathrm{id}_{1_{\mathcal{C}}} \otimes m$, unit $\eta=\mathrm{id}_{1_{\mathcal{C}}} \otimes u$, comonoidal structure given by

$$
\left(? \otimes_{\sigma} A\right)_{2}(X, Y)=\left(\operatorname{id}_{X} \otimes \sigma_{Y} \otimes \operatorname{id}_{A}\right)\left(\operatorname{id}_{X \otimes Y} \otimes \Delta\right) \quad \text { and } \quad\left(? \otimes_{\sigma} A\right)_{0}=\varepsilon,
$$

and left and right antipodes

$$
\begin{aligned}
& s_{X}^{l}=\left(\mathrm{ev}_{A} \otimes \mathrm{id}_{\vee_{X}}\right)\left(\mathrm{id}_{v_{A}} \otimes \sigma \vee_{X}\right)\left(\mathrm{id}_{\vee_{A}} \otimes^{\vee} X X S^{-1}\right), \\
& s_{X}^{r}=\left(\widetilde{\mathrm{ev}}_{A} \otimes \operatorname{id}_{X \vee}\right) \sigma_{A^{\vee} \otimes X^{\vee}}\left(\operatorname{id}_{A^{\vee} \otimes X^{\vee}} \otimes S\right) \text {. }
\end{aligned}
$$

Note that if $A$ is a Hopf algebra in an autonomous braided category $\mathcal{B}$ with braid$\operatorname{ing} \tau$, then $\left(A, \tau_{A,-}\right)$ is a Hopf algebra in $\mathcal{Z}(\mathcal{B}),\left(A, \tau_{-, A}\right)$ is a Hopf algebra in $\mathcal{Z}^{\prime}(\mathcal{B})$, and we have $A \otimes ?=A \otimes_{\tau_{A,-}}$ ? and $? \otimes A=? \otimes_{\tau_{-, A}} A$ as Hopf monads on $\mathcal{B}$.

2.5. Modules over a monad. Let $(T, \mu, \eta)$ be a monad on a category $\mathcal{C}$. An action of $T$ on an object $M$ of $\mathcal{C}$ is a morphism $r: T(M) \rightarrow M$ in $\mathcal{C}$ such that

$$
r T(r)=r \mu_{M} \quad \text { and } \quad r \eta_{M}=\operatorname{id}_{M} .
$$

The pair $(M, r)$ is then called a $T$-module in $\mathcal{C}$, or just a $T$-module 3

Given two $T$-modules $(M, r)$ and $(N, s)$ in $\mathcal{C}$, a morphism of $T$-modules from $(M, r)$ to $(N, r)$ is a morphism $f \in \operatorname{Hom}_{\mathcal{C}}(M, N)$ which is $T$-linear, that is, such that $f r=s T(f)$. This gives rise to the category $T$ - $\mathcal{C}$ of $T$-modules (in $\mathcal{C}$ ), with composition inherited from $\mathcal{C}$. We denote by $U_{T}: T-\mathcal{C} \rightarrow \mathcal{C}$ the forgetful functor of $T$ defined by $U_{T}(M, r)=M$ for any $T$-module $(M, r)$ and $U_{T}(f)=f$ for any $T$-linear morphism $f$.

\footnotetext{
${ }^{3}$ Pairs $(M, r)$ are usually called $T$-algebras in the literature (see Mac98). However, throughout this paper, pairs $(M, r)$ are considered as the analogues of modules over an algebra, and so the term 'algebra' would be awkward in this context.
} 
Example 2.6. Let $A$ be an algebra in a monoidal category $\mathcal{C}$ and consider the monads ? $\otimes A$ and $A \otimes$ ? of Example 2.1. Then the category of (? $\otimes A)$-modules (resp. of $\left(A \otimes\right.$ ?)-modules) coincides with the category $\mathcal{C}_{A}$ of right $A$-modules in $\mathcal{C}$ (resp. with the category ${ }_{A} \mathcal{C}$ of left $A$-modules in $\mathcal{C}$ ):

$$
(? \otimes A)-\mathcal{C}=\mathcal{C}_{A} \quad \text { and } \quad(A \otimes ?)-\mathcal{C}={ }_{A} \mathcal{C} .
$$

2.6. Tannaka dictionary. Structures of bimonad and Hopf monad on a monad $T$ have natural interpretations in terms of the category of $T$-modules:

Theorem 2.7 ([BV07]). Let $T$ be a monad on a monoidal category $\mathcal{C}$ and $T-\mathcal{C}$ be the category of $T$-modules. Then:

(a) If $T$ is a bimonad, then the category $T$ - $\mathcal{C}$ is monoidal by setting

$$
(M, r) \otimes(N, s)=\left(M \otimes N,(r \otimes s) T_{2}(M, N)\right) \quad \text { and } \quad \mathbb{1}_{T-\mathcal{C}}=\left(\mathbb{1}, T_{0}\right) .
$$

Moreover, this gives a bijective correspondence between bimonad structures on the monad $T$ and monoidal structures on $T-\mathcal{C}$ such that the forgetful functor $U_{T}: T-\mathcal{C} \rightarrow \mathcal{C}$ is strict monoidal.

(b) Assume $T$ is a bimonad and $\mathcal{C}$ is left (resp. right) autonomous. Then $T$ has a left (resp. right) antipode if and only if $T$-C is left (resp. right) autonomous. If $s^{l}$ is a left antipode for $T$, left duals in $T-\mathcal{C}$ are given by

$$
{ }^{\vee}(M, r)=\left({ }^{\vee} M, s_{M}^{l} T\left({ }^{\vee} r\right)\right), \quad \mathrm{ev}_{(M, r)}=\mathrm{ev}_{M}, \quad \operatorname{coev}_{(M, r)}=\operatorname{coev}_{M},
$$

and if $s^{r}$ is a right antipode for $T$, right duals in $T$ - $\mathcal{C}$ are given by

$$
(M, r)^{\vee}=\left(M^{\vee}, s_{M}^{r} T\left(r^{\vee}\right)\right), \quad \widetilde{\mathrm{ev}}_{(M, r)}=\widetilde{\mathrm{ev}}_{M}, \quad \widetilde{\operatorname{coev}}_{(M, r)}=\widetilde{\operatorname{coev}}_{M} .
$$

(c) Assume $T$ is a bimonad and $\mathcal{C}$ is autonomous. Then $T$ is a Hopf monad if and only if $T-\mathcal{C}$ is autonomous.

Example 2.8. Let $A$ be a Hopf algebra in a braided autonomous category $\mathcal{B}$ and consider the Hopf monads ? $\otimes A$ and $A \otimes ?$ of Example 2.4. Then

$$
(? \otimes A)-\mathcal{B}=\mathcal{B}_{A} \quad \text { and } \quad(A \otimes ?)-\mathcal{B}={ }_{A} \mathcal{B}
$$

as monoidal categories.

Example 2.9. More generally, let $\mathcal{C}$ be a monoidal category and $(A, \sigma)$ be a Hopf algebra in the braided category $\mathcal{Z}(\mathcal{C})$. Then ${ }_{A} \mathcal{C}$ coincides with the category of modules over the Hopf monad $A \otimes_{\sigma}$ ? on $\mathcal{C}$ defined in Example 2.5. Hence ${ }_{A} \mathcal{C}$ is autonomous, with unit object $(\mathbb{1}, \varepsilon)$ and monoidal product

$$
(M, r) \otimes(N, s)=(r \otimes s)\left(\operatorname{id}_{A} \otimes \sigma_{M} \otimes \operatorname{id}_{N}\right)\left(\Delta \otimes \operatorname{id}_{M \otimes N}\right) .
$$

Likewise, if $(A, \sigma)$ is a Hopf algebra in the braided category $\mathcal{Z}^{\prime}(\mathcal{C})$ (see Remark 1.2), then $\mathcal{C}_{A}$ coincides with the category of modules over the Hopf monad ? $\otimes_{\sigma} A$, and so is autonomous, with a unit object $(\mathbb{1}, \varepsilon)$ and a monoidal product

$$
(M, r) \otimes(N, s)=(r \otimes s)\left(\operatorname{id}_{M} \otimes \sigma_{N} \otimes \operatorname{id}_{A}\right)\left(\operatorname{id}_{M \otimes N} \otimes \Delta\right) .
$$


2.7. Quasitriangular Hopf monads. An R-matrix for a Hopf monad $(T, \mu, \eta)$ on an autonomous category $\mathcal{C}$ is a natural transformation

$$
R=\left\{R_{X, Y}: X \otimes Y \rightarrow T(Y) \otimes T(X)\right\}_{X, Y \in \mathrm{Ob}(\mathcal{C})}
$$

such that, for all objects $X, Y, Z$ of $\mathcal{C}$,

$$
\begin{gathered}
\left(\mu_{Y} \otimes \mu_{X}\right) R_{T(X), T(Y)} T_{2}(X, Y)=\left(\mu_{Y} \otimes \mu_{X}\right) T_{2}(T(Y), T(X)) T\left(R_{X, Y}\right), \\
\left(\operatorname{id}_{T(Z)} \otimes T_{2}(X, Y)\right) R_{X \otimes Y, Z} \\
\quad=\left(\mu_{Z} \otimes \operatorname{id}_{T(X) \otimes T(Y)}\right)\left(R_{X, T(Z)} \otimes \operatorname{id}_{T(Y)}\right)\left(\operatorname{id}_{X} \otimes R_{Y, Z}\right), \\
\left(T_{2}(Y, Z) \otimes \operatorname{id}_{T(X)}\right) R_{X, Y \otimes Z} \\
\quad=\left(\operatorname{id}_{T(Y) \otimes T(Z)} \otimes \mu_{X}\right)\left(\operatorname{id}_{T(Y)} \otimes R_{T(X), Z}\right)\left(R_{X, Y} \otimes \operatorname{id}_{Z}\right), \\
\left(\operatorname{id}_{T(X)} \otimes T_{0}\right) R_{\mathbb{1}, X}=\eta_{X}=\left(T_{0} \otimes \operatorname{id}_{T(X)}\right) R_{X, \mathbb{1}} .
\end{gathered}
$$

A quasitriangular Hopf monad is a Hopf monad equipped with an R-matrix.

Remark 2.10. For a bimonad, an R-matrix is also required to be $*$-invertible (see [BV07, Section 8.2]). For a Hopf monad $T$, this condition is automatic and we have

$$
\begin{aligned}
& R_{X, Y}^{*-1}=\left(\operatorname{id}_{T(X) \otimes T(Y)} \otimes \operatorname{ev}_{X}\left(s_{X}^{l} \otimes \operatorname{id}_{X}\right)\right) \\
& \quad\left(\operatorname{id}_{T(X)} \otimes R \vee_{T(X), Y} \otimes \operatorname{id}_{X}\right)\left(\operatorname{coev}_{T(X)} \otimes \operatorname{id}_{Y \otimes X}\right), \\
&=\left(\widetilde{\operatorname{ev}}_{Y}\left(\operatorname{id}_{Y} \otimes s_{Y}^{r}\right) \otimes \operatorname{id}_{T(X) \otimes T(Y)}\right) \\
& \quad\left(\operatorname{id}_{Y} \otimes R_{X, T(Y)} \otimes \operatorname{id}_{T(Y)}\right)\left(\operatorname{id}_{Y \otimes X} \otimes \widetilde{\left.\operatorname{coev}_{T(Y)}\right)}\right.
\end{aligned}
$$

where $s^{l}$ and $s^{r}$ are the left and right antipodes of $T$.

There is a natural interpretation of R-matrices for a Hopf monad $T$ in terms of braidings on the category of $T$-modules:

Theorem 2.11 ([BV07]). Let $T$ be a Hopf monad on an autonomous category $\mathcal{C}$. Then any $\mathrm{R}$-matrix $R$ for $T$ defines a braiding $\tau$ on the category $T-\mathcal{C}$ as follows:

$$
\tau_{(M, r),(N, s)}=(s \otimes t) R_{M, N}:(M, r) \otimes(N, s) \rightarrow(N, s) \otimes(M, r) .
$$

This assignment is a bijection between $\mathrm{R}$-matrices for $T$ and braidings on $T-\mathcal{C}$.

Remark 2.12. In Section 8.6 we define R-matrices for a Hopf algebra $A$ in a braided autonomous category $\mathcal{B}$ admitting a coend $C$. These R-matrices are morphisms $\mathfrak{r}: C \otimes C \rightarrow A \otimes A$ which encode R-matrices for the Hopf monads ? $\otimes A$ and $A \otimes ?$. They generalize the usual R-matrices for finite-dimensional Hopf algebras.

2.8. Morphisms of Hopf monads. A morphism of monads between two monads $(T, \mu, \eta)$ and $\left(T^{\prime}, \mu^{\prime}, \eta^{\prime}\right)$ on a category $\mathcal{C}$ is a natural transformation $f: T \rightarrow T^{\prime}$ such that, for every object $X$ of $\mathcal{C}$,

$$
f_{X} \mu_{X}=\mu_{X}^{\prime} f_{T^{\prime}(X)} T\left(f_{X}\right) \quad \text { and } \quad f_{X} \eta_{X}=\eta_{X}^{\prime} .
$$

According to [BV07, Lemma 1.7], a morphism of monads $f: T \rightarrow T^{\prime}$ yields a functor $f^{*}: T^{\prime}-\mathcal{C} \rightarrow T-\mathcal{C}$ defined by $f^{*}(M, r)=\left(M, r f_{M}\right)$. Moreover, the mapping $f \mapsto f^{*}$ is a bijective correspondence between: (i) morphisms of monads $f: T \rightarrow T^{\prime}$, and (ii) functors $F: T^{\prime}-\mathcal{C} \rightarrow T-\mathcal{C}$ such that $U_{T} F=U_{T^{\prime}}$.

A morphism of bimonads between two bimonads $T$ and $T^{\prime}$ on a monoidal category $\mathcal{C}$ is a morphism of monads $f: T \rightarrow T^{\prime}$ which is comonoidal, that is,

$$
T_{2}^{\prime}(X, Y) f_{X \otimes Y}=\left(f_{X} \otimes f_{Y}\right) T_{2}(X, Y) \quad \text { and } \quad T_{0}^{\prime} f_{\mathbb{1}}=T_{0} .
$$


According to [BV07, Lemma 2.9], the associated functor $f^{*}: T^{\prime}-\mathcal{C} \rightarrow \mathcal{C} \rightarrow T-\mathcal{C}$ is then monoidal strict. Moreover, the mapping $f \mapsto f^{*}$ is a bijective correspondence between: (i) morphisms of bimonads $f: T \rightarrow T^{\prime}$, and (ii) monoidal functors $F: T^{\prime}-\mathcal{C} \rightarrow T-\mathcal{C}$ such that $U_{T} F=U_{T^{\prime}}$ as monoidal functors.

A morphism of Hopf monads is a morphism of bimonads between Hopf monads.

Example 2.13. Let $A$ be a Hopf algebra in a braided autonomous category $\mathcal{B}$, with braiding $\tau$. Recall that $A^{\text {op }}$ is a Hopf algebra in the mirror $\overline{\mathcal{B}}$ of $\mathcal{B}$. The Hopf monad ? $\otimes A^{\text {op }}$ on $\overline{\mathcal{B}}$ may be seen as a Hopf monad on $\mathcal{B}$. Then

$$
\tau_{A, ?}: A \otimes ? \rightarrow ? \otimes A^{\mathrm{op}}
$$

is an isomorphism of Hopf monads and

$$
\left(\tau_{A, ?}\right)^{*}: \overline{\mathcal{B}}_{A^{\mathrm{op}}}=\left(? \otimes A^{\mathrm{op}}\right)-\mathcal{B} \rightarrow(A \otimes ?)-\mathcal{B}={ }_{A} \mathcal{B}
$$

is an isomorphism of monoidal categories. Likewise, since $\left(A^{\mathrm{op}}\right)^{\mathrm{op}}=A$ as Hopf algebras in $\mathcal{B}, \tau_{?, A}$ induces isomorphisms ? $\otimes A \rightarrow A^{\mathrm{op}} \otimes$ ? and ${ }_{A^{\mathrm{op}}} \overline{\mathcal{B}} \rightarrow \mathcal{B}_{A}$.

\section{Hopf monads, COMONOIDAL ADJUnCTIONS, AND COENDS}

Monads and adjunctions are closely related. This relationship extends naturally to Hopf monads and comonoidal adjunctions between autonomous categories. We show that the forgetful functor of a Hopf monad creates and preserves coends. Lastly, we define the pushforward of a Hopf monad under an adjunction and, as a special case, the cross product of Hopf monads.

3.1. Adjunctions. Let $\mathcal{C}$ and $\mathcal{D}$ be categories. Recall that an adjunction is a pair of functors $(F: \mathcal{C} \rightarrow \mathcal{D}, U: \mathcal{D} \rightarrow \mathcal{C})$ endowed with a bijection

$$
\operatorname{Hom}_{\mathcal{D}}(F(X), Y) \simeq \operatorname{Hom}_{\mathcal{C}}(X, U(Y))
$$

which is natural in both $X \in \mathrm{Ob}(\mathcal{C})$ and $Y \in \mathrm{Ob}(\mathcal{D})$. The functor $F$ is then called a left adjoint of $U$ and the functor $U$ a right adjoint of $F$. Note that a left (resp. right) adjoint of a given functor, if it exists, is unique up to a unique natural isomorphism.

An adjunction $(F, U)$ is entirely determined by two natural transformations $\eta: 1_{\mathcal{C}} \rightarrow U F$ and $\varepsilon: F U \rightarrow 1_{\mathcal{D}}$ satisfying

$$
U(\varepsilon) \eta_{U}=\operatorname{id}_{U} \quad \text { and } \quad \varepsilon_{F} F(\eta)=\operatorname{id}_{F} .
$$

These transformations $\eta$ and $\varepsilon$ are respectively called the unit and counit of the adjunction, and collectively the adjunction morphisms.

Adjunctions may be composed: given two adjunctions $(F: \mathcal{C} \rightarrow \mathcal{D}, U: \mathcal{D} \rightarrow \mathcal{C})$ and $\left(F^{\prime}: \mathcal{D} \rightarrow \mathcal{E}, U^{\prime}: \mathcal{E} \rightarrow \mathcal{D}\right)$, the pair $\left(F^{\prime} F: \mathcal{C} \rightarrow \mathcal{E}, U U^{\prime}: \mathcal{E} \rightarrow \mathcal{C}\right)$ is an adjunction called the composite of $(F, U)$ and $\left(F^{\prime}, U^{\prime}\right)$.

Adjunctions and monads are closely related. Indeed, if $T$ is a monad on a category $\mathcal{C}$, then the forgetful functor $U_{T}: T-\mathcal{C} \rightarrow \mathcal{C}$ has a left adjoint $F_{T}: \mathcal{C} \rightarrow T-\mathcal{C}$, defined by $F_{T}(X)=\left(T(X), \mu_{X}\right)$ for any object $X$ of $\mathcal{C}$ and $F_{T}(f)=T(f)$ for any morphism $f$ in $\mathcal{C}$. The unit of the adjunction $\left(F_{T}, U_{T}\right)$ is the unit $\eta: 1_{\mathcal{C}} \rightarrow T=$ $U_{T} F_{T}$ of the monad $T$, and the counit $\varepsilon: F_{T} U_{T} \rightarrow 1_{T-\mathcal{C}}$ of $\left(F_{T}, U_{T}\right)$ is the $T$-action, that is, $\varepsilon_{(M, r)}=r$ for any $T$-module $(M, r)$.

Moreover, if $(F: \mathcal{C} \rightarrow \mathcal{D}, U: \mathcal{D} \rightarrow \mathcal{C})$ is a pair of adjoint functors, with adjunction morphisms $\eta: 1_{\mathcal{C}} \rightarrow U F$ and $\varepsilon: F U \rightarrow 1_{\mathcal{D}}$, then $T=U F$ is a monad on $\mathcal{C}$ with a product $\mu=U\left(\varepsilon_{F}\right): T^{2} \rightarrow T$ and unit $\eta$. The monad $(T, \mu, \eta)$ is called the monad 
of the adjunction $(F, U)$. In addition, there exists a unique functor $K: \mathcal{D} \rightarrow T$ - $\mathcal{C}$ such that $U_{T} K=U$ and $K F=F_{T}$. The functor $K$ is called the comparison functor and is given by $K(D)=\left(U(D), U\left(\varepsilon_{D}\right)\right)$ for any object $D$ of $\mathcal{D}$.

Note that if $T$ is a monad on $\mathcal{C}$, then $T$ is the monad of the adjunction $\left(F_{T}, U_{T}\right)$ and the comparison functor is the identity functor. In general, however, the comparison functor of an adjunction need not be an equivalence.

3.2. Monadic adjunctions. An adjunction is monadic if its comparison functor (see Section 3.1) is an equivalence. Remark that the composite adjunction of two monadic adjunctions need not be monadic.

A functor $U: \mathcal{D} \rightarrow \mathcal{C}$ is monadic if it admits a left adjoint $F: \mathcal{C} \rightarrow \mathcal{D}$ and the adjunction $(F, U)$ is monadic. If such is the case, the monad $T=U F$ of the adjunction $(F, U)$ is called the monad of $U$. It is well-defined up to a unique isomorphism of monads (as the left adjoint $F$ is unique up to a unique natural isomorphism).

For example, if $T$ is a monad on a category $\mathcal{C}$, the forgetful functor $U_{T}: T-\mathcal{C} \rightarrow \mathcal{C}$ is monadic with monad $T$.

Remark 3.1. Let $U: \mathcal{D} \rightarrow \mathcal{C}$ be a functor. If there exist a monad $T$ on $\mathcal{C}$ and an isomorphism of categories $K: \mathcal{D} \rightarrow T-\mathcal{C}$ such that $U=U_{T} K$, then $F=K^{-1} F_{T}$ is left adjoint to $U$ and the adjunction $(F, U)$ is monadic with monad $T$ and comparison functor $K$.

3.3. Hopf monads and comonoidal adjunctions. A comonoidal adjunction is an adjunction $(F: \mathcal{C} \rightarrow \mathcal{D}, U: \mathcal{D} \rightarrow \mathcal{C})$, where $\mathcal{C}$ and $\mathcal{D}$ are monoidal categories, such that the right adjoint $U: \mathcal{D} \rightarrow \mathcal{C}$ is strong monoidal. In that case, the functor $F$ is comonoidal, with comonoidal structure given by

$$
F_{2}(X, Y)=\varepsilon_{F X \otimes F Y} F U_{2}(F X, F Y) F\left(\eta_{X} \otimes \eta_{Y}\right) \quad \text { and } \quad F_{0}=\varepsilon_{\mathbb{1}} F\left(U_{0}\right),
$$

and the adjunction unit $\eta: 1_{\mathcal{C}} \rightarrow U F$ and counit $\varepsilon: F U \rightarrow 1_{\mathcal{D}}$ are comonoidal natural transformations (viewing $U$ as a strong comonoidal functor).

For example, if $T$ is a bimonad on a monoidal category $\mathcal{C}$, then the adjunction $\left(F_{T}, U_{T}\right)$ is comonoidal.

Remark 3.2. Comonoidal adjunctions are called monoidal adjunctions in BV07. However, it is preferable to call them comonoidal adjunctions since they are nothing but adjunctions $(F, U)$ such that $F$ and $U$ are comonoidal functors and the adjunction unit $\eta: 1_{\mathcal{C}} \rightarrow U F$ and counit $\varepsilon: F U \rightarrow 1_{\mathcal{D}}$ are comonoidal natural transformations. Indeed, in that case, $U$ is a strong comonoidal functor, with $U_{2}(X, Y)^{-1}=U\left(\varepsilon_{X} \otimes \varepsilon_{Y}\right) U F_{2}(U(X), U(Y)) \eta_{U(X) \otimes U(Y)}$, which we may view as a strong monoidal functor.

The monad of a comonoidal adjunction between monoidal categories (resp. autonomous categories) is a bimonad (resp. a Hopf monad). More precisely:

Theorem 3.3 (BV07). Let $(F: \mathcal{C} \rightarrow \mathcal{D}, U: \mathcal{D} \rightarrow \mathcal{C})$ be a comonoidal adjunction between monoidal categories. Denote by $T=U F$ the monad of this adjunction. Then the functor $F$ is comonoidal and $T$ is a bimonad on $\mathcal{C}$. The comparison functor $K: \mathcal{D} \rightarrow T-\mathcal{C}$ is strong monoidal and satisfies $U_{T} K=U$ as monoidal functors and $K F=F_{T}$ as comonoidal functors. If the categories $\mathcal{C}$ and $\mathcal{D}$ are furthermore autonomous, then the bimonad $T$ is a Hopf monad. 
Remark 3.4. Let $(F, U)$ be a comonoidal adjunction between autonomous categories, with unit $\eta$ and counit $\varepsilon$. Let $T=U F$ be the Hopf monad associated with this comonoidal adjunction (see Theorem 3.3). Then the comonoidal structure and antipodes of $T$ are:

$$
\begin{aligned}
& T_{2}(X, Y)=U_{2}(F(X), F(Y))^{-1} U\left(\varepsilon_{F(X) \otimes F(Y)}\right) U F\left(U_{2}(F(X), F(Y))\left(\eta_{X} \otimes \eta_{Y}\right)\right), \\
& T_{0}=U_{0}^{-1} U\left(\varepsilon_{\mathbb{1}}\right) U F\left(U_{0}\right), \\
& s_{X}^{l}={ }^{\vee} \eta_{X} U_{1}^{l}(F(X))^{-1} U\left(\varepsilon^{\vee} F(X)\right) U F\left(U_{1}^{l}(F(X))\right), \\
& s_{X}^{r}=\eta_{X}^{\vee} U_{1}^{r}(F(X))^{-1} U\left(\varepsilon_{F(X)}\right) U F\left(U_{1}^{r}(F(X))\right),
\end{aligned}
$$

where $U_{1}^{l}(Y):{ }^{\vee} U(Y) \rightarrow U\left({ }^{\vee} Y\right)$ and $U_{1}^{r}(Y): U(Y)^{\vee} \rightarrow U\left(Y^{\vee}\right)$ are the compatibility isomorphisms of $U$ with duals (see [BV07, Section 3.2]).

3.4. Hopf monads and right adjoints. If $F: \mathcal{C} \rightarrow \mathcal{D}$ is a functor between autonomous categories, denote by $F^{!}: \mathcal{C} \rightarrow \mathcal{D}$ the functor defined by $F^{!}(X)=F\left({ }^{\vee} X\right)^{\vee}$ and $F^{!}(f)=F\left({ }^{\vee} f\right)^{\vee}$ for all objects $X$ and morphisms $f$ in $\mathcal{C}$.

Lemma 3.5. Let $U: \mathcal{D} \rightarrow \mathcal{C}$ be a strong monoidal functor between autonomous categories. If $F: \mathcal{C} \rightarrow \mathcal{D}$ is a left adjoint for $U$, then $F^{!}$is a right adjoint for $U$.

Proof. Since $U$ is strong monoidal, we have $U\left({ }^{\vee} X\right) \simeq{ }^{\vee} U(X)$ for any object $X$ of $\mathcal{C}$. Hence we have the following isomorphisms:

$$
\begin{aligned}
\operatorname{Hom}_{\mathcal{C}}(U(X), Y) \simeq \operatorname{Hom}_{\mathcal{C}}\left({ }^{\vee} Y,{ }^{\vee} U(X)\right) \simeq \operatorname{Hom}_{\mathcal{C}}\left({ }^{\vee} Y, U\left({ }^{\vee} X\right)\right) \\
\simeq \operatorname{Hom}_{\mathcal{D}}\left(F\left({ }^{\vee} Y\right),{ }^{\vee} X\right) \simeq \operatorname{Hom}_{\mathcal{D}}\left(X, F\left({ }^{\vee} Y\right)^{\vee}\right)=\operatorname{Hom}_{\mathcal{D}}\left(X, F^{!}(Y)\right)
\end{aligned}
$$

which are natural in both $X \in \mathrm{Ob}(\mathcal{C})$ and $Y \in \mathrm{Ob}(\mathcal{D})$.

Proposition 3.6. Let $T$ be a Hopf monad on an autonomous category $\mathcal{C}$. Then:

(a) The endofunctor $T^{!}$of $\mathcal{C}$ is a right adjoint of $T$.

(b) The functor $F_{T}^{!}: \mathcal{C} \rightarrow T-\mathcal{C}$ is a right adjoint of the forgetful functor $U_{T}$.

Proof. Part (a) is [BV07, Corollary 3.12]. Part (b) is Lemma 3.5 applied to the comonoidal adjunction $\left(F_{T}, U_{T}\right)$.

Remark 3.7. If $T$ is a Hopf monad on an autonomous category $\mathcal{C}$, then the adjunction morphisms $e: T T^{!} \rightarrow 1_{\mathcal{C}}$ and $h: 1_{\mathcal{C}} \rightarrow T^{!} T$ are given by $e_{X}=s_{V_{X}}^{r}$ and $h_{X}=\left(s_{X}^{l}\right)^{\vee}$, where $s^{l}$ and $s^{r}$ denote the left and right antipodes of $T$.

Recall that a functor $G: \mathcal{D} \rightarrow \mathcal{C}$ preserves colimits if the image under $G$ of a colimit in $\mathcal{D}$ is a colimit in $\mathcal{C}$. A functor $G: \mathcal{D} \rightarrow \mathcal{C}$ creates colimits if, for any functor $F: I \rightarrow \mathcal{D}$ such that $G F: I \rightarrow \mathcal{C}$ has a colimit, this colimit lifts uniquely to a colimit of $F$. See Mac98 for more precise definitions.

Since the forgetful functor of a monad which preserves colimits creates colimits (by [Bor94, Proposition 4.3.2]), Proposition 3.6 admits the following corollary:

Corollary 3.8. A Hopf monad $T$ on an autonomous category $\mathcal{C}$ preserves colimits, and its forgetful functor $U_{T}: T-\mathcal{C} \rightarrow \mathcal{C}$ creates and preserves colimits. 
3.5. Coends and Hopf monads. Let $\mathcal{C}$ and $\mathcal{D}$ be categories and $F: \mathcal{C}^{\mathrm{op}} \times \mathcal{C} \rightarrow \mathcal{D}$ be a functor. A dinatural transformation $d: F \rightarrow Z$ from $F$ to an object $Z$ of $\mathcal{D}$ is a family $d=\left\{d_{X}: F(X, X) \rightarrow Z\right\}_{X \in \mathrm{Ob}(\mathcal{C})}$ of morphisms in $\mathcal{D}$ satisfying the dinaturality condition

$$
d_{Y} F\left(\operatorname{id}_{Y}, f\right)=F\left(f, \operatorname{id}_{X}\right) d_{X}
$$

for every morphism $f: X \rightarrow Y$ in $\mathcal{C}$. We denote by $\operatorname{DinAT}(F, Z)$ the set of dinatural transformations from $F$ to $Z$.

A coend of a functor $F: \mathcal{C}^{\mathrm{op}} \times \mathcal{C} \rightarrow \mathcal{D}$ consists of an object $C$ of $\mathcal{C}$ and a dinatural transformation $i: F \rightarrow C$ which is universal in the sense that, for every dinatural transformation $d: F \rightarrow Z$, there exists a unique morphism $r: C \rightarrow Z$ such that $d_{X}=r \circ i_{X}$ for all $X \in \mathrm{Ob}(\mathcal{C})$. In other words, the map

$$
\left\{\begin{aligned}
\operatorname{Hom}_{\mathcal{D}}(C, Z) & \rightarrow \operatorname{DinAT}(F, Z) \\
r & \mapsto r i
\end{aligned}\right.
$$

is a bijection. The dinatural transformation $i$ is then called a universal dinatural transformation for $F$. A coend of $F$, if it exists, is unique up to unique isomorphism. Following Mac98, we denote it by $\int^{X \in \mathcal{C}} F(X, X)$.

Coends are well-behaved under adjunction:

Lemma 3.9. Let $\mathcal{C}, \mathcal{D}, \mathcal{E}$ be categories, $(F: \mathcal{C} \rightarrow \mathcal{D}, U: \mathcal{D} \rightarrow \mathcal{C})$ be an adjunction, and $G: \mathcal{D}^{\mathrm{op}} \times \mathcal{C} \rightarrow \mathcal{E}$ be a functor. We have

$$
\int^{X \in \mathcal{C}} G(F(X), X) \simeq \int^{Y \in \mathcal{D}} G(Y, U(Y))
$$

meaning that if either coend exists, then both exist and they are naturally isomorphic.

Proof. Denote by $\eta: 1_{\mathcal{C}} \rightarrow U F$ and $\varepsilon: F U \rightarrow 1_{\mathcal{D}}$ the adjunction morphisms. The lemma results from the existence of a bijection:

$$
\psi: \operatorname{DinAT}\left(G\left(F \times 1_{\mathcal{C}}\right), E\right) \rightarrow \operatorname{Dinat}\left(G\left(1_{\mathcal{D}^{\text {op }}} \times U\right), E\right)
$$

which is natural in $E \in \mathrm{Ob}(\mathcal{E})$. It is defined by $\psi(d)=d_{U} G\left(\varepsilon, \mathrm{id}_{U}\right)$, and its inverse by $\psi^{-1}(t)=t_{F} G\left(\mathrm{id}_{F}, \eta\right)$.

Coends are special cases of colimits (see [Mac98]), and in particular, a functor which preserves (resp. creates) colimits preserves (resp. creates) coends. Hence, by Corollary 3.8

Proposition 3.10. Let $T$ be a Hopf monad on an autonomous category $\mathcal{C}$ and $F: \mathcal{D}^{\mathrm{op}} \times \mathcal{D} \rightarrow T-\mathcal{C}$ be a functor. Then the coend $C=\int^{Y \in \mathcal{D}} U_{T} F(Y, Y)$ exists if and only if the coend $\int^{Y \in \mathcal{D}} F(Y, Y)$ exists. Moreover, given a coend $C=$ $\int^{Y \in \mathcal{D}} U_{T} F(Y, Y)$ with universal dinatural transformation $i_{Y}: U_{T} F(Y, Y) \rightarrow C$, there exists a unique action $r: T(C) \rightarrow C$ of $T$ on $C$ such that $i_{Y}: F(Y, Y) \rightarrow(C, r)$ is $T$-linear. We then have $(C, r)=\int{ }^{Y \in \mathcal{D}} F(Y, Y)$ with universal dinatural transformation $i$. The morphism $r: T(C) \rightarrow C$ is characterized by

$$
r T\left(i_{Y}\right)=i_{Y} \alpha_{Y}, \text { where } F(Y, Y)=\left(U_{T} F(Y, Y), \alpha_{Y}\right),
$$

as $T(i)$ is a universal dinatural transformation. 
3.6. Pushforward of a monad under an adjunction. Let $(F: \mathcal{C} \rightarrow \mathcal{D}, U: \mathcal{D} \rightarrow$ $\mathcal{C}$ ) be an adjunction and $Q$ be an endofunctor of $\mathcal{D}$. The endofunctor $U Q F$ of $\mathcal{C}$ is called the pushforward of $Q$ under the adjunction $(F, U)$ and is denoted by $(F, U)_{*} Q$.

If $Q$ is a monad, then $(F, U)_{*} Q$ is a monad: it is the monad of the composite adjunction $\left(F_{Q} F, U U_{Q}\right)$ of $(F, U)$ and $\left(F_{Q}, U_{Q}\right)$.

If $Q$ is comonoidal and $(F, U)$ is comonoidal, then $(F, U)_{*} Q$ is comonoidal with comonoidal structure the composition of the comonoidal structures of $U_{T}, Q$, and $F_{T}$.

By Theorem 3.3 if the adjunction $(F, U)$ is comonoidal and $Q$ is a bimonad, then $(F, U)_{*} Q$ is a bimonad (since the composite of comonoidal adjunctions is a comonoidal adjunction).

Finally, if $\mathcal{C}$ and $\mathcal{D}$ are autonomous, $(F, U)$ is comonoidal, and $Q$ is a Hopf monad, then $(F, U)_{*} Q$ is a Hopf monad.

Remark 3.11. The structural morphisms of $(F, U)_{*} Q$ can be expressed using those of $Q$ and the adjunction morphisms of $(F, U)$ (by applying Remark 3.4).

3.7. Cross products. Let $T$ be a monad on a category $\mathcal{C}$ and $Q$ be an endofunctor of $T$-C. Denote by $\eta$ and $\varepsilon$ the unit and counit of $\left(U_{T}, F_{T}\right)$. The pushforward of $Q$ under the adjunction $\left(F_{T}, U_{T}\right)$ is called the cross product of $Q$ by $T$ and denoted by $Q \rtimes T$. Recall that $Q \rtimes T=U_{T} Q F_{T}$ as an endofunctor of $\mathcal{C}$.

If $(Q, q, v)$ is a monad on $T$ - $\mathcal{C}$, then $Q \rtimes T$ is a monad on $\mathcal{C}$ with product $p$ and unit $e$ given by

$$
p=q_{F_{T}} Q\left(\varepsilon_{Q F_{T}}\right) \text { and } e=v_{F_{T}} \eta .
$$

If $T$ is a bimonad and $Q$ is comonoidal, then $Q \rtimes T$ is a comonoidal with comonoidal structure given by

$$
\begin{aligned}
& (Q \rtimes T)_{2}(X, Y)=Q_{2}\left(F_{T}(X), F_{T}(Y)\right) Q\left(\varepsilon_{F_{T}(X) \otimes F_{T}(Y)} F_{T}\left(\eta_{X} \otimes \eta_{Y}\right)\right), \\
& (Q \rtimes T)_{0}=Q_{0} Q\left(\varepsilon_{\left(\mathbb{1}, T_{0}\right)}\right) .
\end{aligned}
$$

If $T$ and $Q$ are bimonads, then $Q \rtimes T$ is a bimonad. If $T$ and $Q$ are Hopf monads, then $Q \rtimes T$ is a Hopf monad with left and right antipodes given by

$$
a_{X}^{l}={ }^{\vee} \eta_{X} S_{\vee_{F_{T}}(X)}^{l} Q\left(\varepsilon^{\vee}{{ } F_{T}(X)}\right) \text { and } \quad a_{X}^{r}=\eta_{X}^{\vee} S_{F_{T}^{\vee}(X)}^{r} Q\left(\varepsilon_{Q F_{T}(X) \vee}\right),
$$

where $S^{l}$ and $S^{r}$ are the antipodes of $Q$.

Example 3.12. Let $H$ be a bialgebra over a field $\mathbb{k}$ and $A$ be an $H$-module algebra, that is, an algebra in the monoidal category ${ }_{H}$ Vect $_{\mathrm{k}}$ of left $H$-modules. In this situation, the cross product $A \rtimes H$ (see [Maj95, Proposition 1.6.6]) is the $\mathbb{k}$-algebra $A \rtimes H=A \otimes_{\mathbb{k}} H$ with unit $1_{A} \otimes 1_{H}$ and product given by

$$
(a \otimes h)(b \otimes k)=a\left(h_{(1)} \cdot b\right) \otimes h_{(2)} \cdot k .
$$

Recall that $H \otimes$ ? is a monad on $\operatorname{Vect}_{\mathbb{k}}$ and $A \otimes$ ? is a monad on ${ }_{H}$ Vect $_{\mathbb{k}_{\mathbb{k}}}$. Then

$$
(A \otimes ?) \rtimes(H \otimes ?)=(A \rtimes H) \otimes ?
$$

as monads. Moreover, if $H$ is a quasitriangular bialgebra and $A$ is a $H$-module bialgebra, that is, a bialgebra in the braided category ${ }_{H} \operatorname{Vect}_{\mathbb{k}}$, then $A \rtimes H$ is a 
$\mathbb{k}$-bialgebra with counit $\varepsilon_{A} \otimes \varepsilon_{H}$ and coproduct given by

$$
\Delta(a \otimes h)=\sum_{i} a_{(1)} \otimes t_{i} \cdot h_{(1)} \otimes r_{i} \cdot a_{(2)} \otimes h_{(2)},
$$

where $R=\sum_{i} r_{i} \otimes t_{i}$ is the R-matrix of $H$. In that case $(A \otimes$ ? $) \rtimes(H \otimes ?)=(A \rtimes H) \otimes$ ? as bimonads.

\section{Distributive LAWS AND Liftings}

Given two monads $P$ and $T$ on a category $\mathcal{C}$, when is the composition $P T$ a monad? How can one lift $P$ to a monad on the category $T$ - $\mathcal{C}$ ? Beck's theory of distributive law [Bec69] answers these questions. In this section, we recall the basic results of this theory and extend them to Hopf monads.

4.1. Distributive laws between algebras. Let $(A, m, u)$ and $(B, \mu, \eta)$ be two algebras in a monoidal category $\mathcal{C}$. Given a morphism $\Omega: B \otimes A \rightarrow A \otimes B$ in $\mathcal{C}$, set

$$
p=(m \otimes \mu)\left(\operatorname{id}_{A} \otimes \Omega \otimes \operatorname{id}_{B}\right):(A \otimes B) \otimes(A \otimes B) \rightarrow(A \otimes B) .
$$

Then $(A \otimes B, p, u \otimes \eta)$ is an algebra in $\mathcal{C}$ if and only if $\Omega$ satisfies

$$
\begin{aligned}
& \Omega\left(\operatorname{id}_{B} \otimes m\right)=\left(m \otimes \operatorname{id}_{B}\right)\left(\operatorname{id}_{A} \otimes \Omega\right)\left(\Omega \otimes \operatorname{id}_{A}\right), \quad \Omega\left(\operatorname{id}_{B} \otimes u\right)=u \otimes \operatorname{id}_{B}, \\
& \Omega\left(\mu \otimes \mathrm{id}_{A}\right)=\left(\mathrm{id}_{A} \otimes \mu\right)\left(\Omega \otimes \mathrm{id}_{B}\right)\left(\mathrm{id}_{B} \otimes \Omega\right), \quad \Omega\left(\eta \otimes \mathrm{id}_{A}\right)=\mathrm{id}_{A} \otimes \eta .
\end{aligned}
$$

If such is the case, we say that $\Omega$ is a distributive law of $B$ over $A$. The algebra $(A \otimes B, p, u \otimes \eta)$ is then denoted by $A \otimes_{\Omega} B$. Note that $i=\left(\operatorname{id}_{A} \otimes \eta\right): A \rightarrow A \otimes_{\Omega} B$ and $j=\left(u \otimes \operatorname{id}_{B}\right): B \rightarrow A \otimes_{\Omega} B$ are algebra morphisms and that the middle unitary law holds:

$$
p\left(\mathrm{id}_{A} \otimes \eta \otimes u \otimes \mathrm{id}_{A}\right)=\mathrm{id}_{A \otimes B} .
$$

In other words, we have $p(i \otimes j)=\mathrm{id}_{A \otimes B}$.

Remark 4.1. Let $(C, p, e)$ be an algebra in $\mathcal{C}$ and $i: A \rightarrow C, j: B \rightarrow C$ be two algebra morphisms such that $\Theta=p(i \otimes j): A \otimes B \rightarrow C$ is an isomorphism in $\mathcal{C}$. Then there exists a unique distributive law $\Omega$ of $B$ over $A$ such that $\Theta$ is an algebra isomorphism from $A \otimes_{\Omega} B$ to $C$. Moreover:

$$
\Omega=\Theta^{-1} p(j \otimes i), \quad i=\Theta\left(\operatorname{id}_{A} \otimes \eta\right) \quad \text { and } \quad j=\Theta\left(u \otimes \operatorname{id}_{B}\right) .
$$

Remark 4.2. If a distributive law $\Omega: B \otimes A \rightarrow A \otimes B$ of $B$ over $A$ is an isomorphism, then $\Omega^{-1}$ is a distributive law of $A$ over $B$ and $\Omega: B \otimes_{\Omega^{-1}} A \rightarrow A \otimes_{\Omega} B$ is an isomorphism of algebras.

Example 4.3. Let $A$ and $B$ be bialgebras in a braided category $\mathcal{B}$. A distributive law of $B$ over $A$ is comultiplicative if it satisfies

$$
\begin{aligned}
& \left(\operatorname{id}_{A} \otimes \tau_{A, B} \otimes \operatorname{id}_{B}\right)\left(\Delta_{A} \otimes \Delta_{B}\right) \Omega=(\Omega \otimes \Omega)\left(\operatorname{id}_{B} \otimes \tau_{B, A} \otimes \operatorname{id}_{A}\right)\left(\Delta_{B} \otimes \Delta_{A}\right), \\
& \left(\varepsilon_{A} \otimes \varepsilon_{B}\right) \Omega=\varepsilon_{B} \otimes \varepsilon_{A},
\end{aligned}
$$

where $\tau$ is the braiding of $\mathcal{B}$. A comultiplicative distributive law is nothing but a distributive law between algebras in the monoidal category of coalgebras in $\mathcal{B}$. Let $\Omega$ be a comultiplicative distributive law of $B$ over $A$. Then $A \otimes_{\Omega} B$ is a bialgebra 
in $\mathcal{B}$. Furthermore, if $A$ and $B$ are Hopf algebras, then $A \otimes_{\Omega} B$ is a Hopf algebra with structural morphisms:

$$
\begin{array}{ll}
m_{A \otimes_{\Omega} B}=\left(m_{A} \otimes m_{B}\right)\left(\operatorname{id}_{A} \otimes \Omega \otimes i_{B}\right), & u_{A \otimes_{\Omega} B}=u_{A} \otimes u_{B}, \\
\Delta_{A \otimes_{\Omega} B}=\left(\operatorname{id}_{A} \otimes \tau_{A, B} \otimes i_{B}\right)\left(\Delta_{A} \otimes \Delta_{B}\right), & \varepsilon_{A \otimes_{\Omega} B}=\varepsilon_{A} \otimes \varepsilon_{B}, \\
S_{A \otimes_{\Omega} B}=S_{A} \otimes S_{B}, &
\end{array}
$$

where $m_{C}, u_{C}, \Delta_{C}, \varepsilon_{C}, S_{C}$ denote respectively the product, unit, coproduct, counit, and antipode of a Hopf algebra $C$.

4.2. Lifting monads and bimonads. Let $(P, m, u)$ be a monad on a category $\mathcal{C}$ and $U: \mathcal{D} \rightarrow \mathcal{C}$ be a functor. A lift of the monad $P$ to $\mathcal{D}$ is a monad $(\tilde{P}, \tilde{m}, \tilde{u})$ on $\mathcal{D}$ such that $P U=U \tilde{P}, m_{U}=U(\tilde{m})$, and $u_{U}=U(\tilde{u})$.

Let $P$ be a bimonad on a monoidal category $\mathcal{C}$ and $U: \mathcal{D} \rightarrow \mathcal{C}$ be a strong monoidal functor. A lift of the bimonad $P$ to $\mathcal{D}$ is bimonad $\tilde{P}$ on $\mathcal{D}$ which is a lift of the monad $P$ to $\mathcal{D}$ such that $U \tilde{P}=P U$ as comonoidal functors.

4.3. Distributive laws between monads. Let $(T, \mu, \eta)$ and $(P, m, u)$ be monads on a category $\mathcal{C}$. Following Beck Bec69, a distributive law of $T$ over $P$ is a natural transformation $\Omega: T P \rightarrow P T$ verifying

$$
\begin{array}{ll}
\Omega_{X} T\left(m_{X}\right)=m_{T(X)} P\left(\Omega_{X}\right) \Omega_{P(X)}, & \Omega_{X} T\left(u_{X}\right)=u_{T(X)}, \\
\Omega_{X} \mu_{P(X)}=P\left(\mu_{X}\right) \Omega_{T(X)} T\left(\Omega_{X}\right), & \Omega_{X} \eta_{P(X)}=P\left(\eta_{X}\right),
\end{array}
$$

for all object $X$ of $\mathcal{C}$.

Remark 4.4. Viewing the monads $T$ and $P$ as algebras in the monoidal category of endofunctors of $\mathcal{C}$ (with monoidal product $\circ$ and unit object $1_{\mathcal{C}}$ ), the above definition of a distributive law agrees with that given in Section 4.1.

Let $\Omega$ be a distributive law of $T$ over $P$. First, $\Omega$ defines a monad structure on the endofunctor $P T$ of $\mathcal{C}$, with product $p$ and unit $e$ given by

$$
p_{X}=m_{T(X)} P^{2}\left(\mu_{X}\right) P\left(\Omega_{T(X)}\right) \quad \text { and } \quad e_{X}=u_{T(X)} \eta_{X} .
$$

The monad $(P T, p, e)$ is denoted by $P \circ_{\Omega} T$. Second, $\Omega$ defines a lift $\left(\tilde{P}^{\Omega}, \tilde{m}, \tilde{u}\right)$ of the monad $P$ to the category $T-\mathcal{C}$ as follows:

$$
\tilde{P}^{\Omega}(M, r)=\left(P(M), P(r) \Omega_{M}\right), \quad \tilde{m}_{(M, r)}=m_{M}, \quad \text { and } \quad \tilde{u}_{(M, r)}=u_{M} .
$$

Furthermore, there is a canonical isomorphism of categories

$$
K:\left\{\begin{array}{llc}
\tilde{P}^{\Omega}-(T-\mathcal{C}) & \longrightarrow & \left(P \circ_{\Omega} T\right)-\mathcal{C} \\
((M, r), s) & \longmapsto & \left(M, U_{T}(s) P(r)\right)
\end{array}\right.
$$

with inverse

$$
K^{-1}:\left\{\begin{array}{ccc}
\left(P \circ_{\Omega} T\right)-\mathcal{C} & \longrightarrow & \tilde{P}_{-}(T-\mathcal{C}) \\
(A, \alpha) & \longmapsto & \left(\left(A, \alpha u_{T(A)}\right), \alpha P\left(\eta_{A}\right)\right)
\end{array} .\right.
$$

In fact $K$ is the comparison functor of the composite adjunction

$$
\tilde{P}^{\Omega}-(T-\mathcal{C}) \underset{F_{\tilde{P} \Omega}}{\stackrel{U_{\tilde{P} \Omega}}{\rightleftharpoons}} T-\mathcal{C} \underset{F_{T}}{\stackrel{U_{T}}{\longrightarrow}} \mathcal{C} .
$$

Thus this composite adjunction is monadic with $\operatorname{monad} P \circ_{\Omega} T$. 
The assignments $\Omega \mapsto P \circ_{\Omega} T$ and $\Omega \mapsto \tilde{P}^{\Omega}$ are one-to-one in the following sense:

Theorem 4.5 ([Bec69]). Let $(T, \mu, \eta)$ and $(P, m, u)$ be monads on a category $\mathcal{C}$. We have bijective correspondences between:

(i) distributive laws $\Omega: T P \rightarrow P T$ of $T$ over $P$;

(ii) products $p: P T P T \rightarrow P T$ for which:

(a) $\left(P T, p, u_{T} \eta\right)$ is a monad on $\mathcal{C}$;

(b) $u_{T}: T \rightarrow P T$ and $P(\eta): P \rightarrow P T$ are morphisms of monads;

(c) the middle unitary law $p_{X} P\left(\eta_{P T(X)} u_{T(X)}\right)=\mathrm{id}_{P T(X)}$ holds;

(iii) lifts of the monad $P$ on $\mathcal{C}$ to a monad $\tilde{P}$ on $T-\mathcal{C}$.

4.4. Distributive laws between bimonads. Let $T$ and $P$ be bimonads on a monoidal category $\mathcal{C}$. Recall that $T P$ and $P T$ are comonoidal endofunctors of $\mathcal{C}$. A distributive law $\Omega: T P \rightarrow P T$ of $T$ over $P$ is comonoidal if it is comonoidal as a natural transformation, that is, if it satisfies

$$
(P T)_{2}(X, Y) \Omega_{X \otimes Y}=\left(\Omega_{X} \otimes \Omega_{Y}\right)(T P)_{2}(X, Y) \quad \text { and } \quad(P T)_{0} \Omega_{\mathbb{1}}=(T P)_{0} .
$$

Remark 4.6. Viewing the bimonads $T$ and $P$ as algebras in the monoidal category of comonoidal endofunctors of $\mathcal{C}$ (see Remark 2.2), a comonoidal distributive law is a distributive law in the sense of Section 4.1 .

Beck's Theorem 4.5 was generalized by Street Str72] to monads in a 2-category. Applying this theorem to the case of the 2-category of monoidal categories and comonoidal functors, we obtain:

Theorem 4.7. Let $(T, \mu, \eta)$ and $(P, m, u)$ be bimonads on a monoidal category $\mathcal{C}$. We have bijective correspondences between:

(i) comonoidal distributive laws $\Omega: T P \rightarrow P T$ of $T$ over $P$;

(ii) products $p: P T P T \rightarrow P T$ for which:

(a) $\left(P T, p, u_{T} \eta\right)$ is a bimonad on $\mathcal{C}$;

(b) $u_{T}: T \rightarrow P T$ and $P(\eta): P \rightarrow P T$ are morphisms of bimonads;

(c) the middle unitary law $p_{X} P\left(\eta_{P T(X)} u_{T(X)}\right)=\mathrm{id}_{P T(X)}$ holds;

(iii) Lifts of the bimonad $P$ on $\mathcal{C}$ to a bimonad $\tilde{P}$ on $T-\mathcal{C}$.

Also, if $\Omega$ is a comonoidal distributive law of $T$ over $P$, the canonical isomorphism of categories $\tilde{P}^{\Omega}-(T-\mathcal{C}) \simeq\left(P \circ_{\Omega} T\right)-\mathcal{C}$ is strict monoidal.

Example 4.8. Let $\mathcal{B}$ be a braided category, $A$ and $B$ be two bialgebras in $\mathcal{B}$, and $\Omega: B \otimes A \rightarrow A \otimes B$ be a morphism in $\mathcal{B}$. Then the following conditions are equivalent:

(i) $\Omega \otimes \mathrm{id}_{1_{\mathcal{B}}}$ is a comonoidal distributive law of $B \otimes$ ? over $A \otimes$ ?;

(ii) $\operatorname{id}_{1_{\mathcal{B}}} \otimes \Omega$ is a comonoidal distributive law of ? $\otimes A$ over ? $\otimes B$;

(iii) $\Omega$ is a comultiplicative distributive law of $B$ over $A$ (see Example 4.3). If such is the case, we have the following equalities of bimonads:

$$
\begin{aligned}
(A \otimes ?) \circ_{\left(\Omega \otimes \mathrm{id}_{1_{\mathcal{B}}}\right)}(B \otimes ?) & =\left(A \otimes_{\Omega} B\right) \otimes ?, \\
(? \otimes B) \circ_{\left(\mathrm{id}_{\mathcal{B}_{\mathcal{B}}} \otimes \Omega\right)}(? \otimes A) & =? \otimes\left(A \otimes_{\Omega} B\right) .
\end{aligned}
$$

Remark 4.9. Let $\Omega: T P \rightarrow P T$ be a distributive law between monads on a category $\mathcal{C}$. Then $\tilde{P}^{\Omega} \rtimes T=P \circ_{\Omega} T$ as monads, where $\rtimes$ denotes the cross product (see Section 3.7). Moreover, if $\mathcal{C}$ is monoidal, $T$ and $P$ are bimonads, and $\Omega$ is comonoidal, then $\tilde{P}^{\Omega} \rtimes T=P \circ_{\Omega} T$ as bimonads. 
4.5. Distributive laws and antipodes. We show here that if $\Omega: T P \rightarrow P T$ is a comonoidal distributive law between Hopf monads, then the composition $P{ }_{\Omega} T$ and the lift $\tilde{P}^{\Omega}$ are Hopf monads:

Proposition 4.10. Let $T$ and $P$ be bimonads on a monoidal category $\mathcal{C}$ and let $\Omega: T P \rightarrow P T$ be a comonoidal distributive law of $T$ over $P$. Then:

(a) If $\mathcal{C}$ is left autonomous, $T$ has a left antipode $s^{l}$, and $P$ has a left antipode $S^{l}$, then the bimonads $P \circ_{\Omega} T$ and $\tilde{P}^{\Omega}$ have left antipodes, denoted by $a^{l}$ and $\tilde{S}^{l}$ respectively, given by

$$
\begin{aligned}
& a_{X}^{l}=S_{X}^{l} P\left(s_{P(X)}^{l}\right) P T\left({ }^{\vee} \Omega_{X}\right): P T\left({ }^{\vee} P T(X)\right) \rightarrow{ }^{\vee} X, \\
& \tilde{S}_{(M, r)}^{l}=S_{M}^{l}: \tilde{P}^{\Omega}\left({ }^{\vee} \tilde{P}^{\Omega}(M, r)\right) \rightarrow{ }^{\vee}(M, r) .
\end{aligned}
$$

(b) If $\mathcal{C}$ is right autonomous, $T$ has a right antipode $s^{r}$, and $P$ has a right antipode $S^{r}$, then the bimonads $P \circ_{\Omega} T$ and $\tilde{P}^{\Omega}$ have right antipodes, denoted by $a^{r}$ and $\tilde{S}^{r}$ respectively, given by

$$
\begin{aligned}
& a_{X}^{r}=S_{X}^{r} P\left(s_{P(X)}^{r}\right) \operatorname{PT}\left(\Omega_{X}^{\vee}\right): \operatorname{PT}\left(\operatorname{PT}(X)^{\vee}\right) \rightarrow X^{\vee}, \\
& \tilde{S}_{(M, r)}^{r}=S_{M}^{r}: \tilde{P}^{\Omega}\left(\tilde{P}^{\Omega}(M, r)^{\vee}\right) \rightarrow(M, r)^{\vee} .
\end{aligned}
$$

Proof. Let us prove part (a). One first checks that $a_{X}^{l}$ satisfies the axioms of a left antipode, that is,

$$
\begin{aligned}
(P T)_{0} P T & \left(\mathrm{ev}_{X}\right) P T\left({ }^{\vee}\left(u_{T(X)} \eta_{X}\right) \otimes \operatorname{id}_{X}\right) \\
& =\operatorname{ev}_{P T(X)}\left(a_{P T(X)}^{l} P T\left({ }^{\vee} p_{X}\right) \otimes \operatorname{id}_{P T(X)}\right)(P T)_{2}\left({ }^{\vee} P T(X), X\right), \\
\left(u_{T(X)} \eta_{X}\right. & \left.\otimes \operatorname{id}_{\vee_{X}}\right) \operatorname{coev}_{X}(P T)_{0} \\
& =\left(p_{X} \otimes a_{X}^{l}\right)(P T)_{2}\left(P T(X),{ }^{\vee} P T(X)\right) P T\left(\operatorname{coev}_{P T(X)}\right) .
\end{aligned}
$$

This can be done by applying the axioms for the left antipodes $s^{l}$ and $S^{l}$ of $T$ and $P$ and the axioms for the distributive law $\Omega$. By Theorem 2.7(b), this implies that $\left(P \circ_{\Omega} T\right)-\mathcal{C}$ is left autonomous. Now recall that

$$
K:\left\{\begin{array}{lll}
\tilde{P}^{\Omega}-(T-\mathcal{C}) & \stackrel{\sim}{\longrightarrow}\left(P \circ_{\Omega} T\right)-\mathcal{C} \\
((M, r), s) & \longmapsto & (M, s P(r))
\end{array}\right.
$$

is a strict monoidal isomorphism of categories (see Section 4.3). Therefore $\tilde{P}^{\Omega}-(T-\mathcal{C})$ is left autonomous and so, by Theorem [2.7(b), $\tilde{P}^{\Omega}$ has a left antipode $\tilde{S}^{l}$. Furthermore, given a $\tilde{P}^{\Omega}$-module $((M, r), s)$, we have

$$
K^{-1}\left({ }^{\vee} K((M, r), s)\right)=\left(\left({ }^{\vee} M, s_{M}^{l} T(r)\right), \rho\right),
$$

where $U_{T}(\rho)=a_{M}^{l} P T\left({ }^{\vee} P(r)^{\vee} s\right) P\left(\eta_{M}\right)=S_{M}^{l} P\left({ }^{\vee} s\right)$. Hence $\tilde{S}_{(M, r)}^{l}=S_{M}^{l}$.

Part (b) results from part (a) applied to the coopposite Hopf monads

$$
\left(P \circ_{\Omega} T\right)^{\mathrm{cop}}=P^{\mathrm{cop}} \circ_{\Omega} T^{\mathrm{cop}} \text { and }\left(\tilde{P}^{\Omega}\right)^{\mathrm{cop}}=\left(\widetilde{P^{\mathrm{cop}}}\right)^{\Omega},
$$

see Remark 2.3 .

From Proposition 4.10, Theorem 4.7 and Remark 4.9, we deduce:

Corollary 4.11. If $T$ and $P$ are Hopf monads on an autonomous category $\mathcal{C}$ and $\Omega: T P \rightarrow P T$ is a comonoidal distributive law, then $P \circ_{\Omega} T$ is a Hopf monad on $\mathcal{C}$, $\tilde{P}^{\Omega}$ is a Hopf monad on $T-\mathcal{C}$, and $\tilde{P}^{\Omega} \rtimes T=P \circ_{\Omega} T$ as Hopf monads. 
4.6. Invertible distributive laws. Let $T, P$ be two monads on a category $\mathcal{C}$ and $\Omega: T P \rightarrow P T$ be an invertible distributive law of $T$ over $P$. Then $\Omega^{-1}: P T \rightarrow T P$ is a distributive law of $P$ over $T$ and $\Omega$ is a isomorphism of monads from $T \circ_{\Omega^{-1}} P$ to $P \circ_{\Omega} T$.

If $\mathcal{C}$ is monoidal, $P, T$ are bimonads, and $\Omega: T P \rightarrow P T$ is a comonoidal distributive law of $T$ on $P$, then $\Omega^{-1}: P T \rightarrow T P$ is a comonoidal distributive law of $P$ over $T$ and $\Omega$ is a isomorphism of bimonads from $T \circ_{\Omega^{-1}} P$ to $P \circ_{\Omega} T$.

Proposition 4.12. Let $P, T$ be Hopf monads on an autonomous category $\mathcal{C}$. Then any comonoidal distributive law $\Omega: T P \rightarrow P T$ of $T$ over $P$ is invertible. Furthermore, for any object $X$ of $\mathcal{C}$, we have

$$
\Omega_{X}^{-1}=S_{\vee_{T P(X)}^{r}}^{r} P\left(s_{P\left({ }^{\vee} T P(X)\right)}^{r}\right) P T\left(\Omega_{\vee_{T P(X)}}\right) P T\left(P\left(s_{P(X)}^{l}\right)^{\vee}\right) P T\left(S_{X}^{l \vee}\right),
$$

where $s^{l}, s^{r}, S^{l}, S^{r}$ denote left and right antipodes of $T$ and $P$, respectively.

Proof. The functors $T, P$ and $P T$ are Hopf monads by assumption and Corollary 4.11. Therefore, by Proposition 3.6, the functors $T^{!}, P^{!}$, and $(P T)^{!}$are right adjoints for $T, P$, and $P T$, respectively. On the other hand, by composition of adjunctions, $P^{!} \circ T^{!}=(P T)^{!}$is a right adjoint for $T P$. As a left adjoint is unique up to a unique natural isomorphism, we obtain a canonical isomorphism $\alpha: P T \rightarrow T P$. Denoting $e: T T^{!} \rightarrow 1_{\mathcal{C}}, h: 1_{\mathcal{C}} \rightarrow T^{!} T, e^{\prime}: P P^{!} \rightarrow 1_{\mathcal{C}}, h^{\prime}: 1_{\mathcal{C}} \rightarrow P^{!} P$, $E: P T(P T)^{!} \rightarrow 1_{\mathcal{C}}$, and $H: 1_{\mathcal{C}} \rightarrow(P T)^{!} P T$ the adjunction morphisms, we have $\alpha=E_{T P} P T P^{!}\left(h_{P}\right) P T\left(h^{\prime}\right)$. Now the adjunction morphisms can be expressed in terms of the antipodes, see Remark 3.7. Therefore, using Proposition 4.10, we get that, for any object $X$ of $\mathcal{C}$,

$$
\alpha_{X}=S_{\vee_{T P(X)}^{r}}^{r} P\left(s_{P\left({ }^{\vee} T P(X)\right)}^{r}\right) P T\left(\Omega_{\vee_{T P(X)}}^{\vee} P T\left(P\left(s_{P(X)}^{l}\right)^{\vee}\right) P T\left(S_{X}^{l \vee}\right) .\right.
$$

Furthermore, $E_{X}=S_{\vee_{X}}^{r} P\left(s_{P\left({ }^{\vee} X\right)}^{r}\right) P T\left(\Omega_{\vee_{X}}^{\vee}\right)$ and $H_{X}=(P T)^{!}\left(\Omega_{X}\right) P\left(s_{P(X)}^{l}\right)^{\vee} S_{X}^{l \vee}$. Hence

$$
\begin{aligned}
\operatorname{id}_{P T(X)} & =E_{P T(X)} P T\left(H_{X}\right) \\
& =E_{P T(X)} P T(P T)^{!}\left(\Omega_{X}\right) P T\left(P\left(s_{P(X)}^{l}\right)^{\vee}\right) P T\left(S_{X}^{l \vee}\right) \\
& =\Omega_{X} E_{T P(X)} P T\left(P\left(s_{P(X)}^{l}\right)^{\vee}\right) P T\left(S_{X}^{l \vee}\right) \quad \text { by functoriality of } E \\
& =\Omega_{X} S_{\vee_{T P(X)}^{r}} P\left(s_{P\left({ }^{r} T P(X)\right)}^{r}\right) P T\left(\Omega_{\vee_{T P(X)}}^{\vee}\right) P T\left(P\left(s_{P(X)}^{l}\right)^{\vee}\right) P T\left(S_{X}^{l \vee}\right) \\
& =\Omega_{X} \alpha_{X} .
\end{aligned}
$$

This shows that $\Omega$, as the inverse of the isomorphism $\alpha$, is an isomorphism.

Remark 4.13. Let $\Omega: B \otimes A \rightarrow A \otimes B$ be a distributive law between two Hopf algebras $A$ and $B$ in a braided autonomous category $\mathcal{B}$ with braiding $\tau$. Then, applying Proposition 4.12 to the distributive law of Example 4.8, we find that $\Omega$ is invertible and its inverse is given by

$$
\Omega^{-1}=\left(S_{B}^{-1} \otimes S_{A}^{-1}\right) \tau_{B, A}^{-1} \Omega \tau_{A, B}\left(S_{A} \otimes S_{B}\right),
$$

where $S_{A}$ and $S_{B}$ are the antipodes of $A$ and $B$.

\section{The Centralizer of a Hopf monad}

In this section, we introduce the notion of centralizer of a Hopf monad and interpret its category of modules as the categorical center relative to the Hopf monad. 
5.1. Centralizers of endofunctors. Let $\mathcal{C}$ be a monoidal category and $T$ be an endofunctor of $\mathcal{C}$.

A centralizer of $T$ at an object $X$ of $\mathcal{C}$ is a pair $(Z, \delta)$, where $Z \in \mathrm{Ob}(\mathcal{C})$ and

$$
\delta=\left\{\delta_{Y}: X \otimes Y \rightarrow T(Y) \otimes Z\right\}_{Y \in \mathrm{Ob}(\mathcal{C})}: X \otimes 1_{\mathcal{C}} \rightarrow T \otimes Z
$$

is a natural transformation, verifying the following universal property: for every object $W$ of $\mathcal{C}$ and every natural transformation $\xi: X \otimes 1_{\mathcal{C}} \rightarrow T \otimes W$, there exists a unique morphism $r: Z \rightarrow W$ in $\mathcal{C}$ such that $\xi=\left(\operatorname{id}_{T} \otimes r\right) \delta$. Note that a centralizer of $T$ at $X$, if it exists, is unique up to a unique isomorphism.

Remark 5.1. The notion of a centralizer is not invariant under left/right symmetry. We should properly call it a 'left-handed' centralizer. We can as well define a 'right-handed' centralizer of $T$ at $X$ to be a pair $\left(Z^{\prime}, \delta^{\prime}\right)$, with

$$
\delta^{\prime}=\left\{\delta_{Y}^{\prime}: Y \otimes X \rightarrow Z^{\prime} \otimes T(Y)\right\}_{Y \in \mathrm{Ob}(\mathcal{C})}: 1_{\mathcal{C}} \otimes X \rightarrow Z^{\prime} \otimes T
$$

satisfying the relevant universal property. Note that this is equivalent to saying that $\left(Z^{\prime}, \delta^{\prime}\right)$ is a 'left-handed' centralizer of $T$ at $X$ in the monoidal category $\mathcal{C}^{\otimes 0 p}$. By left/right symmetry, all notions and results concerning 'left-handed' centralizers can be adapted to the 'right-handed' version.

The endofunctor $T$ is said to be centralizable at an object $X$ of $\mathcal{C}$ if it admits a centralizer at $X$.

A centralizer of $T$ is a pair $\left(Z_{T}, \partial\right)$, where $Z_{T}$ is an endofunctor of $\mathcal{C}$ and

$$
\partial=\left\{\partial_{X, Y}: X \otimes Y \rightarrow T(Y) \otimes Z_{T}(X)\right\}_{X, Y \in \operatorname{Ob}(\mathcal{C})}: \otimes \rightarrow\left(T \otimes Z_{T}\right) \sigma_{\mathcal{C}, \mathcal{C}}
$$

is a natural transformation such that $\left(Z_{T}(X), \partial_{X, 1_{\mathcal{C}}}\right)$ is a centralizer of $T$ at $X$ for every object $X$ of $\mathcal{C}$.

The endofunctor $T$ is said to be centralizable if it admits a centralizer. An endofunctor of $\mathcal{C}$ is centralizable if and only if it is centralizable at every object of $\mathcal{C}$. In that case, its centralizer is essentially unique. More precisely:

Lemma 5.2. Let $T$ be an endofunctor of a monoidal category $\mathcal{C}$. We have:

(a) Given a centralizer $\left(Z_{T}(X), \partial_{X}\right)$ of $T$ at every object $X$ of $\mathcal{C}$, the assignment $Z_{T}: X \mapsto Z_{T}(X)$ admits a unique structure of functor such that

$$
\partial=\left\{\partial_{X, Y}=\left(\partial_{X}\right)_{Y}: X \otimes Y \rightarrow T(Y) \otimes Z_{T}(X)\right\}_{X, Y \in \operatorname{Ob}(\mathcal{C})}
$$

is a natural transformation. The pair $\left(Z_{T}, \partial\right)$ is then a centralizer of $T$.

(b) If $(Z, \partial)$ and $\left(Z^{\prime}, \partial^{\prime}\right)$ are centralizers of $T$, then there exists a unique natural isomorphism $\alpha: Z \rightarrow Z^{\prime}$ such that $\partial^{\prime}=\left(\operatorname{id}_{T} \otimes \alpha\right) \partial$.

Proof. For each morphism $f: X \rightarrow X^{\prime}$ in $\mathcal{C}$, by the universal property of centralizers there exists a unique morphism $Z_{T}(f): Z_{T}(X) \rightarrow Z_{T}\left(X^{\prime}\right)$ such that

$$
\left(\operatorname{id}_{T} \otimes Z_{T}(f)\right) \partial_{X, 1_{\mathcal{C}}}=\partial_{X^{\prime}, 1_{\mathcal{C}}}\left(f \otimes 1_{\mathcal{C}}\right),
$$

and this assignment defines the only structure of functor on $Z_{T}$ such that $\partial$ is a natural transformation. 
5.2. Centralizers and coends. In this section, we give a characterization of centralizable endofunctors in a left autonomous category in terms of coends.

Proposition 5.3. Let $\mathcal{C}$ be a left autonomous category, $T$ be an endofunctor of $\mathcal{C}$, and $X$ be an object of $\mathcal{C}$. Then $T$ is centralizable at $X$ if and only if the coend

$$
Z_{T}(X)=\int^{Y \in \mathcal{C}}{ }^{\vee} T(Y) \otimes X \otimes Y
$$

exists. If such is the case, denoting by $i$ the universal dinatural transformation of the coend and setting

$$
\left(\partial_{X}\right)_{Y}=\left(\operatorname{id}_{T(Y)} \otimes i_{Y}\right)\left(\operatorname{coev}_{T(Y)} \otimes \mathrm{id}_{X \otimes Y}\right),
$$

the pair $\left(Z_{T}(X), \partial_{X}\right)$ is a centralizer of $T$ at $X$.

Proof. Let $F: \mathcal{C}^{\text {op }} \times \mathcal{C} \rightarrow \mathcal{C}$ be the functor defined by $F(Y, Z)={ }^{\vee} T(Y) \otimes X \otimes Z$ and $F(f, g)={ }^{\vee} T(f) \otimes X \otimes g$. By duality, we have a bijection

$$
\psi: \operatorname{Dinat}(F, Z) \rightarrow \operatorname{Hom}\left(X \otimes 1_{\mathcal{C}}, T \otimes Z\right)
$$

which is natural in $Z \in \mathrm{Ob}(\mathcal{C})$. It is defined by

$$
\psi(j)_{Y}=\left(\operatorname{id}_{T(Y)} \otimes j_{Y}\right)\left(\operatorname{coev}_{T(Y)} \otimes \operatorname{id}_{X \otimes Y}\right): X \otimes Y \rightarrow T(Y) \otimes Z
$$

and its inverse by

$$
\psi^{-1}(\delta)_{Y}=\left(\operatorname{ev}_{T(Y)} \otimes \operatorname{id}_{Z}\right)\left(\operatorname{id}_{T(Y)} \otimes \delta_{Y}\right):{ }^{\vee} T(Y) \otimes X \otimes Y \rightarrow Z .
$$

Therefore $T$ is centralizable at $X$ if and only if $F$ admits a coend and, if so, the centralizer of $T$ at $X$ is canonically isomorphic to the coend of $F$.

5.3. Extended factorization property of the centralizer. Let $T$ be a centralizable endofunctor of a monoidal category $\mathcal{C}$ and $\left(Z_{T}, \partial\right)$ be a centralizer of $T$. For any non-negative integer $n$, let

$$
\partial^{n}: \otimes_{n+1} \rightarrow\left(T^{\otimes n} \otimes Z_{T}^{n}\right) \sigma_{\mathcal{C}, \mathcal{C}^{n}}
$$

be the natural transformation defined by the following diagram:

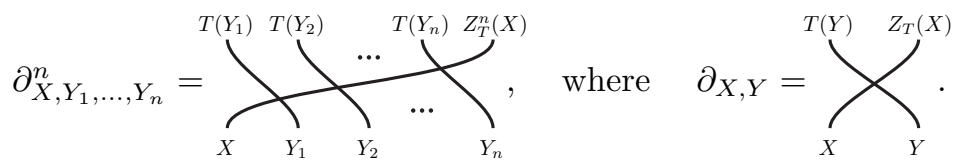

In other words, the morphisms

$$
\partial_{X, Y_{1}, \ldots, Y_{n}}^{n}: X \otimes Y_{1} \otimes \cdots \otimes Y_{n} \rightarrow T\left(Y_{1}\right) \otimes \cdots \otimes T\left(Y_{n}\right) \otimes Z_{T}^{n}(X)
$$

are defined inductively by $\partial_{X}^{0}=\mathrm{id}_{X}$ and

$$
\partial_{X, Y_{1}, \ldots, Y_{n+1}}^{n+1}=\left(\operatorname{id}_{T\left(Y_{1}\right) \otimes \cdots \otimes T\left(Y_{n}\right)} \otimes \partial_{Z_{T}^{n}(X), Y_{n+1}}\right)\left(\partial_{X, Y_{1}, \ldots, Y_{n}}^{n} \otimes \operatorname{id}_{Y_{n+1}}\right) .
$$

Notice $\partial^{1}=\partial$ and $\partial^{p+q}=\left(\operatorname{id}_{T \otimes p} \otimes \partial^{q}\right)\left(\partial^{p} \otimes \operatorname{id}_{\otimes_{q}}\right)$ for all non-negative integers $p, q$.

Lemma 5.4. Assume $\mathcal{C}$ is left autonomous. Let $\mathcal{D}$ be category and $K, L: \mathcal{D} \rightarrow \mathcal{C}$ be two functors. For every non-negative integer $n$ and every natural transformation $\xi: K \otimes \otimes_{n} \rightarrow\left(T^{\otimes n} \otimes L\right) \sigma_{\mathcal{D}, \mathcal{C}^{n}}$, there exists a unique natural transformation 

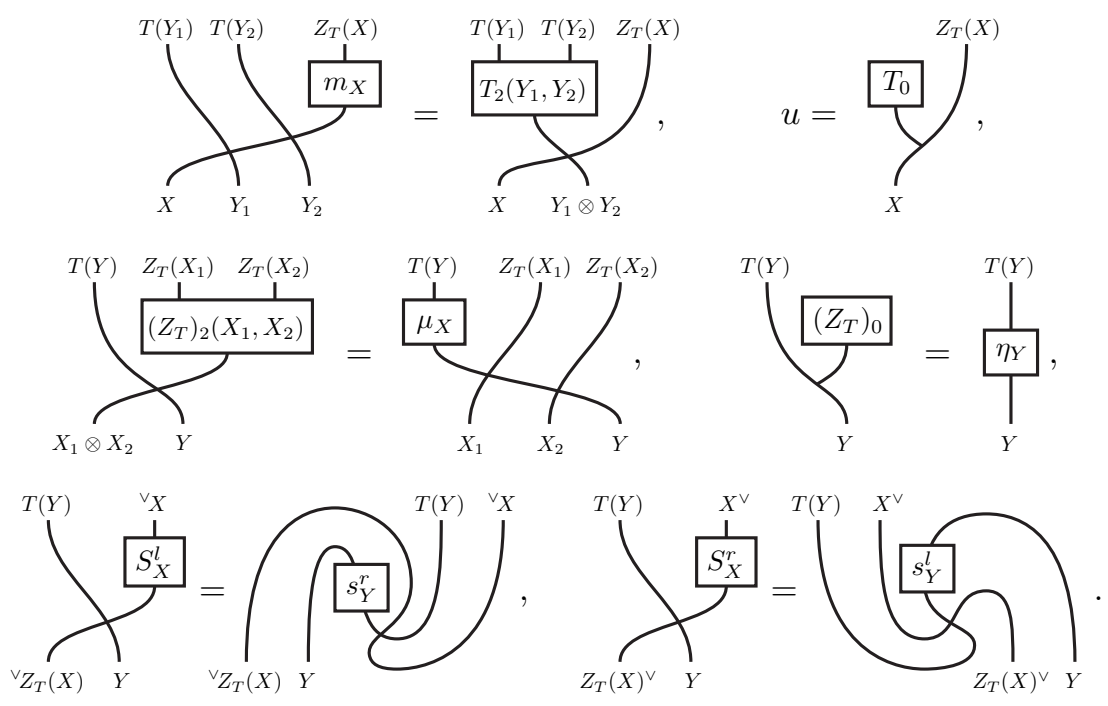

FiguRE 1. Structural morphisms of $Z_{T}$

$r: Z_{T}^{n} K \rightarrow L$ such that

$$
\left(\operatorname{id}_{T\left(Y_{1}\right) \otimes \cdots \otimes T\left(Y_{n}\right)} \otimes r_{X}\right) \partial_{X, Y_{1}, \ldots, Y_{n}}^{n}=\xi_{X, Y_{1}, \ldots, Y_{n}},
$$

that is,

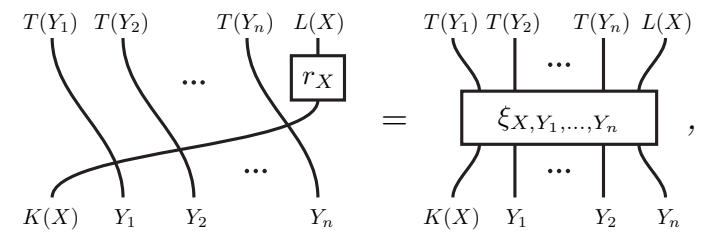

for all $X \in \mathrm{Ob}(\mathcal{D})$ and $Y_{1}, \ldots, Y_{n} \in \mathrm{Ob}(\mathcal{C})$.

Remark 5.5. We will often write the equality defining $r$ in Lemma 5.4 as

$$
\left(\mathrm{id}_{T \otimes n} \otimes r\right) \partial_{K, 1_{\mathcal{C}}}^{n}=\xi .
$$

Strictly speaking, it should be $\left(\mathrm{id}_{T^{\otimes n}} \otimes r\right) \sigma_{\mathcal{D}, \mathcal{C}^{n}} \partial_{K, 1_{\mathcal{C}^{n}}}^{n}=\xi$. However, in this kind of formula, we will usually omit the permutation $\sigma$, as it can easily be recovered from the context.

Proof of Lemma 5.4. The lemma can be verified by induction on $n$ using the Parameter Theorem and Fubini Theorem for coends (see [Mac98]) and the fact that, by Proposition 5.3, we have $Z_{T} K(X)=\int^{Y \in \mathcal{C} \vee} T(Y) \otimes K(X) \otimes Y$ for all $X \in \mathrm{Ob}(\mathcal{C})$.

5.4. Structure of centralizers. In this section, we show that the centralizer $Z_{T}$ of a Hopf monad $T$ is a Hopf monad. The structural morphisms of $Z_{T}$ are defined as in Figure 1 using the extended factorization property of $Z_{T}$ given in Lemma 5.4. More precisely:

Theorem 5.6. Let $T$ be a centralizable endofunctor of a left autonomous category $\mathcal{C}$ and let $\left(Z_{T}, \partial\right)$ be its centralizer. We have: 
(a) If $T$ is comonoidal, then $Z_{T}$ is a monad on $\mathcal{C}$, with product $m: Z_{T}^{2} \rightarrow Z_{T}$ and unit $u: 1_{\mathcal{C}} \rightarrow Z_{T}$ defined by

$$
\left(\mathrm{id}_{T \otimes 2} \otimes m\right) \partial^{2}=\left(T_{2} \otimes \mathrm{id}_{Z_{T}}\right) \partial_{1_{\mathcal{C},} \otimes} \quad \text { and } \quad u=\left(T_{0} \otimes \mathrm{id}_{Z_{T}}\right) \partial_{1_{\mathcal{C}}, \mathbb{1}} .
$$

(b) If $(T, \mu, \eta)$ is a monad, then $Z_{T}$ is comonoidal, with comonoidal structure defined by

$$
\begin{aligned}
& \left(\operatorname{id}_{T} \otimes\left(Z_{T}\right)_{2}\right) \partial_{\otimes, 1_{\mathcal{C}}}=\left(\mu \otimes \operatorname{id}_{Z_{T}^{\otimes 2}}\right)\left(\partial_{1_{\mathcal{C}}, T} \otimes \mathrm{id}_{Z_{T}}\right)\left(\operatorname{id}_{1_{\mathcal{C}}} \otimes \partial\right), \\
& \left(\operatorname{id}_{T} \otimes\left(Z_{T}\right)_{0}\right) \partial_{\mathbb{1}, 1_{\mathcal{C}}}=\eta .
\end{aligned}
$$

(c) If $T$ is a bimonad, then $Z_{T}$ is a bimonad on $\mathcal{C}$, with the monad structure of part (a) and the comonoidal structure of part (b).

(d) If $\mathcal{C}$ is autonomous, $T$ is a bimonad, and $T$ has a right antipode $s^{r}$, then the bimonad $Z_{T}$ has a left antipode $S^{l}$ defined by

$$
\left(\operatorname{id}_{T} \otimes S^{l}\right) \partial{ }^{\vee} Z_{T}, 1_{\mathcal{C}}={ }^{\vee}\left(\left(s^{r} \otimes \operatorname{id}_{Z_{T}}\right) \partial_{1_{\mathcal{C}}, T^{\vee}}\right) .
$$

(e) If $\mathcal{C}$ is autonomous, $T$ is a bimonad, and $T$ has a left antipode $s^{l}$, then the bimonad $Z_{T}$ has a right antipode $S^{r}$ defined by

$$
\left(\operatorname{id}_{T} \otimes S^{r}\right) \partial_{Z_{T}^{\vee}, 1_{\mathcal{C}}}=\left(\left(s^{l} \otimes \operatorname{id}_{Z_{T}}\right) \partial_{1_{\mathcal{C}}, \vee_{T}}\right)^{\vee} .
$$

In particular, if $\mathcal{C}$ is autonomous and $T$ is a Hopf monad, then $Z_{T}$ is a Hopf monad.

Remark 5.7. The centralizer construction $T \mapsto Z_{T}$ is functorial, contravariant in $T$. More precisely, let $\mathcal{C}$ be a left autonomous category and $T, T^{\prime}$ be two centralizable endofunctors of $\mathcal{C}$, with centralizers $\left(Z_{T}, \partial\right)$ and $\left(Z_{T^{\prime}}, \partial^{\prime}\right)$, respectively. Then, for each natural transformation $f: T \rightarrow T^{\prime}$, there exists a unique natural transformation $Z_{f}: Z_{T^{\prime}} \rightarrow Z_{T}$ such that

$$
\left(\mathrm{id}_{T^{\prime}} \otimes Z_{f}\right) \partial^{\prime}=\left(f \otimes \mathrm{id}_{Z_{T}}\right) \partial .
$$

We have $Z_{f g}=Z_{g} Z_{f}$ and $Z_{\mathrm{id}_{T}}=\mathrm{id}_{Z_{T}}$. Moreover, if $f$ is comonoidal, then $Z_{f}$ is a morphism of monads. If $f$ if a morphism of monads, then $Z_{f}$ is comonoidal. Thus, if $f$ is a morphism of bimonads or Hopf monads, so is $Z_{f}$.

Remark 5.8. Let $T$ be a centralizable Hopf monad on an autonomous category $\mathcal{C}$, with centralizer $\left(Z_{T}, \partial\right)$. Set

$$
\partial_{X, Y}^{\prime}=\left(\left.s_{X}^{s_{Y}^{r}}\right|_{Y} ^{Z_{T}(X)}: Y \otimes X \rightarrow Z_{T}(X) \otimes T(Y)\right.
$$

and $Z_{T^{\mathrm{cop}}}=\left(Z_{T}\right)^{\mathrm{cop}}$. Then $\left(Z_{T^{\mathrm{cop}}}, \partial^{\prime}\right)$ is a centralizer of $T^{\mathrm{cop}}$ in $\mathcal{C}^{\otimes \mathrm{op}}$. Moreover, $Z_{T^{\text {cop }}}=\left(Z_{T}\right)^{\text {cop }}$ as Hopf monads when $Z_{T}$ and $Z_{T^{\text {cop }}}$ are equipped with the Hopf monad structure of Theorem 5.6. In the language of Remark [5.1, 'left centralizability' and 'right centralizability' are equivalent for a Hopf monad $T$, and a 'left-handed' centralizer $Z_{T}^{\prime}=\left(Z_{T^{\mathrm{cop}}}\right)^{\mathrm{cop}}$ can be identified with a 'right-handed' centralizer $Z_{T}$ in a manner preserving the Hopf monad structures. 
Proof of Theorem [5.6. To simplify notation, set $Z=Z_{T}$. Let us prove part (a). By definition of the product $m$ and unit $u$ of $Z$, we have

$$
\begin{aligned}
& \left(\mathrm{id}_{T \otimes 3} \otimes m Z(m)\right) \partial^{3}=\left(T_{2} \otimes \mathrm{id}_{T} \otimes m\right) \partial_{1_{\mathcal{C}}, \otimes, 1_{\mathcal{C}}}^{2} \\
& =\left(\left(T_{2} \otimes \mathrm{id}_{T}\right) T_{2} \otimes \mathrm{id}_{Z}\right) \partial_{1_{\mathcal{C}}, \otimes_{2}} \\
& =\left(\left(\mathrm{id}_{T} \otimes T_{2}\right) T_{2} \otimes \mathrm{id}_{Z}\right) \partial_{1_{\mathcal{C}}, \otimes_{2}} \\
& =\left(\mathrm{id}_{T} \otimes T_{2} \otimes m\right) \partial_{1_{\mathcal{C}}, 1_{\mathcal{C}}, \otimes}^{2} \\
& =\left(\mathrm{id}_{T \otimes 3} \otimes m m_{Z}\right) \partial^{3} \text {. }
\end{aligned}
$$

Therefore $m Z(m)=m m_{Z}$ by the uniqueness assertion of Lemma 5.4, Likewise, since

$$
\begin{aligned}
\left(\operatorname{id}_{T} \otimes m Z(u)\right) \partial & =\left(\operatorname{id}_{T} \otimes m\right) \partial_{Z, 1_{\mathcal{C}}}\left(u \otimes \operatorname{id}_{1_{\mathcal{C}}}\right) \\
& =\left(T_{0} \otimes \mathrm{id}_{T} \otimes m\right) \partial_{1_{\mathcal{C}}, \mathbb{1}, 1_{\mathcal{C}}}^{2} \\
& =\left(\left(T_{0} \otimes \mathrm{id}_{T}\right) T_{2}(\mathbb{1},-) \otimes \mathrm{id}_{Z}\right) \partial \\
& =\left(\mathrm{id}_{T} \otimes \mathrm{id}_{Z}\right) \partial
\end{aligned}
$$

and

$$
\begin{aligned}
\left(\mathrm{id}_{T} \otimes m u_{Z}\right) \partial & =\left(\mathrm{id}_{T} \otimes T_{0} \otimes m\right) \partial_{1_{\mathcal{C}}, 1_{\mathcal{C}}, \mathbb{1}}^{2} \\
& =\left(\left(\mathrm{id}_{T} \otimes T_{0}\right) T_{2}(-, \mathbb{1}) \otimes \mathrm{id}_{Z}\right) \partial \\
& =\left(\operatorname{id}_{T} \otimes \operatorname{id}_{Z}\right) \partial,
\end{aligned}
$$

we get $m Z(u)=\operatorname{id}_{Z}=m u_{Z}$. Hence $(Z, m, u)$ is a monad on $\mathcal{C}$.

Let us prove part (b). By definition of the natural transformation $Z_{2}$, we have

$$
\begin{aligned}
\left(\operatorname{id}_{T}\right. & \left.\otimes\left(\operatorname{id}_{Z} \otimes Z_{2}\right) Z_{2}\right) \partial_{\otimes_{3}, 1_{\mathcal{C}}} \\
& =\left(\mu T(\mu) \otimes \mathrm{id}_{Z^{\otimes 3}}\right)\left(\partial_{T^{2}, 1_{\mathcal{C}}} \otimes \mathrm{id}_{Z^{\otimes 2}}\right)\left(\mathrm{id}_{1_{\mathcal{C}}} \otimes \partial_{T, 1_{\mathcal{C}}} \otimes \mathrm{id}_{Z}\right)\left(\mathrm{id}_{\otimes} \otimes \partial\right) \\
& =\left(\mu \mu_{T} \otimes \mathrm{id}_{Z^{\otimes 3}}\right)\left(\partial_{T^{2}, 1_{\mathcal{C}}} \otimes \mathrm{id}_{Z^{\otimes 2}}\right)\left(\mathrm{id}_{1_{\mathcal{C}}} \otimes \partial_{T, 1_{\mathcal{C}}} \otimes \mathrm{id}_{Z}\right)\left(\mathrm{id}_{\otimes} \otimes \partial\right) \\
& =\left(\mathrm{id}_{T} \otimes\left(Z_{2} \otimes \mathrm{id}_{Z}\right) Z_{2}\right) \partial_{\otimes_{3}, 1_{\mathcal{C}}},
\end{aligned}
$$

and so $\left(\operatorname{id}_{Z} \otimes Z_{2}\right) Z_{2}=\left(Z_{2} \otimes \operatorname{id}_{Z}\right) Z_{2}$ by Lemma 5.4. Likewise, since

$$
\begin{gathered}
\left(\operatorname{id}_{T} \otimes\left(\operatorname{id}_{Z} \otimes Z_{0}\right) Z_{2}(-, \mathbb{1})\right) \partial=\left(\mu \otimes \operatorname{id}_{Z} \otimes Z_{0}\right)\left(\partial_{1_{\mathcal{C}}, T} \otimes \operatorname{id}_{Z(\mathbb{1})}\right)\left(\operatorname{id}_{1_{\mathcal{C}}} \otimes \partial_{\mathbb{1}, 1_{\mathcal{C}}}\right) \\
=\left(\mu \otimes \operatorname{id}_{Z}\right) \partial_{1_{\mathcal{C}}, T}\left(\operatorname{id}_{1_{\mathcal{C}}} \otimes \eta\right)=\left(\mu T(\eta) \otimes \operatorname{id}_{Z}\right) \partial=\partial
\end{gathered}
$$

and

$$
\begin{aligned}
\left(\operatorname{id}_{T} \otimes\left(Z_{0} \otimes \operatorname{id}_{Z}\right) Z_{2}(\mathbb{1},-)\right) \partial & =\left(\mu \otimes Z_{0} \otimes \operatorname{id}_{Z}\right)\left(\partial_{\mathbb{1}, T} \otimes \operatorname{id}_{Z}\right) \partial \\
& =\left(\mu \eta_{T} \otimes \operatorname{id}_{Z}\right) \partial=\partial,
\end{aligned}
$$

we get $\left(\operatorname{id}_{Z} \otimes Z_{0}\right) Z_{2}\left(1_{\mathcal{C}}, \mathbb{1}\right)=\operatorname{id}_{Z}=\left(Z_{0} \otimes \operatorname{id}_{Z}\right) Z_{2}\left(\mathbb{1}, 1_{\mathcal{C}}\right)$. Hence $Z$ is a comonoidal functor.

Let us prove part (c). We have to show that $m$ and $u$ are comonoidal morphisms. Since $\mu$ and $\eta$ are comonoidal, we have

$$
\begin{aligned}
\left(\mathrm{id}_{T^{\otimes 2}} \otimes Z_{2} m\right) \partial_{\otimes, 1_{\mathcal{C}}, 1_{\mathcal{C}}}^{2} & =\left(T_{2} \mu \otimes \mathrm{id}_{Z_{\otimes 2}}\right)\left(\partial_{1_{\mathcal{C}}, T} \otimes \mathrm{id}_{Z}\right)\left(\mathrm{id}_{1_{\mathcal{C}}} \otimes \partial\right) \\
& =\left((\mu \otimes \mu) T_{2} T\left(T_{2}\right) \otimes \mathrm{id}_{Z \otimes 2}\right)\left(\partial_{1_{\mathcal{C}}, T} \otimes \mathrm{id}_{Z}\right)\left(\mathrm{id}_{1_{\mathcal{C}}} \otimes \partial\right) \\
& =\left(\operatorname{id}_{T^{\otimes 2}} \otimes(m \otimes m) Z_{2} Z\left(Z_{2}\right)\right) \partial_{\otimes, 1_{\mathcal{C}}, 1_{\mathcal{C}}}^{2}
\end{aligned}
$$


and $\left(\mathrm{id}_{T^{\otimes 2}} \otimes Z_{0} m_{\mathbb{1}}\right) \partial_{1,1_{\mathcal{c}, 1} 1_{\mathcal{C}}}^{2}=T_{2} \eta=\eta \otimes \eta=\left(\mathrm{id}_{T^{\otimes 2}} \otimes Z_{0} Z\left(Z_{0}\right)\right) \partial_{1,1_{\mathcal{c},} 1_{\mathcal{C}}}^{2}$. Therefore $Z_{2} m=(m \otimes m) Z_{2} Z\left(Z_{2}\right)$ and $Z_{0} m_{\mathbb{1}}=Z_{0} Z\left(Z_{0}\right)$ by Lemma [5.4, that is, $m$ is comonoidal. Moreover,

$$
\begin{aligned}
Z_{2} u & =\left(T_{0} \otimes Z_{2}\right) \partial_{\otimes, \mathbb{1}}=\left(T_{0} \mu_{\mathbb{1}} \otimes \mathrm{id}_{Z^{\otimes 2}}\right) \partial_{1_{\mathcal{C}}, 1_{\mathcal{C}}, \mathbb{1}}^{2} \\
& =\left(T_{0} T\left(T_{0}\right) \otimes \operatorname{id}_{Z^{\otimes 2}}\right) \partial_{1_{\mathcal{C}, 1}, 1_{\mathcal{C}} \mathbb{1}}^{2}=u \otimes u
\end{aligned}
$$

and $Z_{0} u_{\mathbb{1}}=\left(T_{0} \otimes Z_{0}\right) \partial_{\mathbb{1}, \mathbb{1}}=T_{0} \eta_{\mathbb{1}}=\mathrm{id}_{\mathbb{1}}$. Hence $u$ is comonoidal.

Parts (d) and (e) can be proved in a similar way, but we will instead deduce them in Section 5.8 from the next theorem, Theorem 5.12

5.5. Categorical center relative to a Hopf monad. Let $T$ be a comonoidal endofunctor of a monoidal category $\mathcal{C}$. The center of $\mathcal{C}$ relative to $T$, or for short the $T$-center of $\mathcal{C}$, is the category $\mathcal{Z}_{T}(\mathcal{C})$ defined as follows: objects are pairs $(M, \sigma)$, where $M$ is an object of $\mathcal{C}$ and $\sigma: M \otimes 1_{\mathcal{C}} \rightarrow T \otimes M$ is a natural transformation, such that

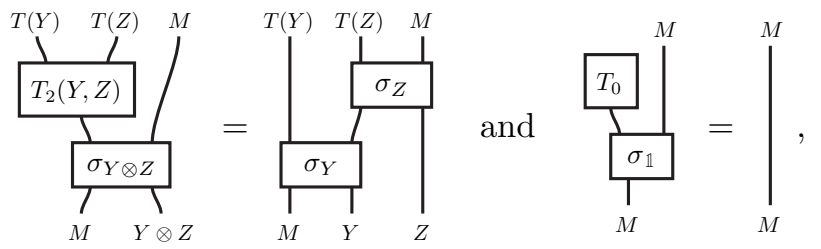

that is,

$$
\begin{aligned}
& \left(T_{2}(Y, Z) \otimes \operatorname{id}_{M}\right) \sigma_{Y \otimes Z}=\left(\operatorname{id}_{T(Y)} \otimes \sigma_{Z}\right)\left(\sigma_{Y} \otimes \operatorname{id}_{Z}\right) \quad \text { for all } Y, Z \in \operatorname{Ob}(\mathcal{C}), \\
& \left(T_{0} \otimes \operatorname{id}_{M}\right) \sigma_{\mathbb{1}}=\operatorname{id}_{M} .
\end{aligned}
$$

A morphism $f:(M, \sigma) \rightarrow\left(M^{\prime}, \sigma^{\prime}\right)$ is a morphism $f: M \rightarrow M^{\prime}$ in $\mathcal{C}$ such that

$$
\left(\operatorname{id}_{T(Y)} \otimes f\right) \sigma_{Y}=\sigma_{Y}^{\prime}\left(f \otimes \operatorname{id}_{Y}\right)
$$

for every object $Y$ of $\mathcal{C}$. The composition and identities are inherited from $\mathcal{C}$.

Let $\mathcal{U}_{T}: \mathcal{Z}_{T}(\mathcal{C}) \rightarrow \mathcal{C}$ be the forgetful functor defined by

$$
\mathcal{U}_{T}(M, \sigma)=M \quad \text { and } \quad \mathcal{U}_{T}(f)=f .
$$

If $\mathcal{C}$ is autonomous and $T$ is a Hopf monad, then $\mathcal{Z}_{T}(\mathcal{C})$ is autonomous. More precisely:

Proposition 5.9. Let $(T, \mu, \eta)$ be a bimonad on a monoidal category $\mathcal{C}$. Then $\mathcal{Z}_{T}(\mathcal{C})$ is monoidal, with monoidal product $(M, \sigma) \otimes(N, \gamma)=(M \otimes N, \rho)$ and unit object $(\mathbb{1}, \eta)$, where

$$
\rho_{Y}=\left(\mu_{Y} \otimes \operatorname{id}_{M \otimes N}\right)\left(\sigma_{T(Y)} \otimes \operatorname{id}_{N}\right)\left(i d_{M} \otimes \gamma_{Y}\right)=\underbrace{\sigma_{T}}_{M}
$$

and the forgetful functor $\mathcal{U}_{T}: \mathcal{Z}_{T}(\mathcal{C}) \rightarrow \mathcal{C}$ is strict monoidal. Now assume $\mathcal{C}$ is autonomous. If $T$ has a right antipode $s^{r}$, then $\mathcal{Z}_{T}(\mathcal{C})$ is left autonomous and left 
duals are given by ${ }^{\vee}(M, \sigma)=\left({ }^{\vee} M, \sigma^{l}\right)$, where

$$
\sigma_{Y}^{l}=\vee^{\vee}\left(\left(s_{Y}^{r} \otimes \operatorname{id}_{M}\right) \sigma_{T(Y)^{\vee}}\right)=\overbrace{\vee_{M}}^{s_{Y}^{r}}
$$

with left evaluation $\mathrm{ev}_{M}$ and left coevaluation $\operatorname{coev}_{M}$. If $T$ has a left antipode $s^{l}$, then $\mathcal{Z}_{T}(\mathcal{C})$ is right autonomous and right duals are given by $(M, \sigma)^{\vee}=\left(M^{\vee}, \sigma^{r}\right)$, where

$$
\sigma_{Y}^{r}=\left(\left(s_{Y}^{l} \otimes \operatorname{id}_{M}\right) \sigma^{\vee_{T(Y)}}\right)^{\vee}=(\left.\overbrace{M^{\vee}}^{T(Y)}\right|_{Y} ^{M^{\vee}},
$$

with right evaluation $\widetilde{\mathrm{ev}}_{M}$ and right coevaluation $\widetilde{\operatorname{cov}}_{M}$. In particular, if $T$ is a Hopf monad, then the category $\mathcal{Z}_{T}(\mathcal{C})$ is autonomous.

Proof. The fact that $(M \otimes N, \rho)$ and $(\mathbb{1}, \eta)$ are objects of $\mathcal{Z}_{T}(\mathcal{C})$ results from the comonoidality of $\mu$ and $\eta$. The associativity and unitarity of the monoidal product of $\mathcal{Z}_{T}(\mathcal{C})$ follows from the associativity of $\mu$ and unitarity of $\eta$, and its functoriality from the definition of morphisms of $\mathcal{Z}_{T}(\mathcal{C})$.

Assume $\mathcal{C}$ is autonomous and $T$ has a right antipode $s^{r}$. The fact that $\left({ }^{\vee} M, \sigma^{l}\right)$ is an object of $\mathcal{Z}_{T}(\mathcal{C})$ results from the anti-comultiplicativity of $s^{r}$ (see Section 2.3). The axioms of a right antipode imply that the left evaluation $\mathrm{ev}_{M}$ and left coevaluation $\operatorname{coev}_{M}$ are morphisms of $\mathcal{Z}_{T}(\mathcal{C})$. Thus $\left({ }^{\vee} M, \sigma^{l}\right)$ is a left dual of $(M, \sigma)$. Likewise, if $T$ has a left antipode, $\left(M^{\vee}, \sigma^{r}\right)$ is a right dual of $(M, \sigma)$.

Remark 5.10. If $\mathcal{C}$ is an autonomous category, then $\mathcal{Z}_{1_{\mathcal{C}}}(\mathcal{C})$ coincides with the usual center $\mathcal{Z}(\mathcal{C})$ of $\mathcal{C}$ (see Section 1.10).

Remark 5.11. The definition of the category $\mathcal{Z}_{T}(\mathcal{C})$ is not left/right symmetric. One may also consider the category $\mathcal{Z}_{T}^{\prime}(\mathcal{C})=\mathcal{Z}_{T^{\mathrm{cop}}}\left(\mathcal{C}^{\otimes \mathrm{op}}\right)^{\otimes \mathrm{op}}$ whose objects are pairs $(M, \sigma)$, where $M$ is an object of $\mathcal{C}$ and $\sigma: 1_{\mathcal{C}} \otimes M \rightarrow M \otimes T$ is a natural transformation satisfying the obvious conditions. If $\mathcal{C}$ is autonomous and $T$ is a Hopf monad, then the category $\mathcal{Z}_{T}^{\prime}(\mathcal{C})$ is autonomous and isomorphic to $\mathcal{Z}_{T}(\mathcal{C})$ via the strict monoidal functor $\mathcal{Z}_{T}(\mathcal{C}) \rightarrow \mathcal{Z}_{T}^{\prime}(\mathcal{C})$ defined by $(M, \sigma) \mapsto\left(M, \sigma^{\prime}\right)$, where

$$
\sigma_{Y}^{\prime}=\left(\widetilde{\operatorname{ev}}_{Y}\left(\operatorname{id}_{Y} \otimes s_{Y}^{r}\right) \otimes \operatorname{id}_{M \otimes T(Y)}\right)\left(\operatorname{id}_{Y} \otimes \sigma_{T(Y)} \otimes \operatorname{id}_{T(Y)}\right)\left(\operatorname{id}_{Y \otimes M} \otimes \widetilde{\operatorname{coev}}_{T(Y)}\right) .
$$

In particular, $\mathcal{Z}_{1_{\mathcal{C}}}^{\prime}(\mathcal{C})=\mathcal{Z}^{\prime}(\mathcal{C})$, see Remark 1.2

5.6. Monadicity of centers. In this section, we show that the center relative to a centralizable Hopf monad is monoidally equivalent to the category of modules of its centralizer.

Theorem 5.12. Let $T$ be a centralizable comonoidal endofunctor of a left autonomous category $\mathcal{C}$, with centralizer $\left(Z_{T}, \partial\right)$. The functor $E: Z_{T}-\mathcal{C} \rightarrow \mathcal{Z}_{T}(\mathcal{C})$, defined by

$$
E(M, r)=\left(M,\left(\operatorname{id}_{T} \otimes r\right) \partial_{M, 1_{\mathcal{C}}}\right) \quad \text { and } \quad E(f)=f,
$$


is an isomorphism of categories such that the following triangle commutes:

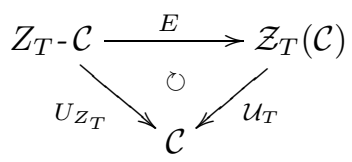

Furthermore, if $T$ is a bimonad (which implies that $Z_{T}$ is a bimonad and $\mathcal{Z}_{T}(\mathcal{C})$ is monoidal), then $E$ is strict monoidal (and so $\mathcal{U}_{T} E=U_{Z_{T}}$ as monoidal functors).

We prove Theorem 5.12 in Section 5.7

Remark 5.13. The functor $\mathcal{F}_{T}=E F_{Z_{T}}: \mathcal{C} \rightarrow \mathcal{Z}_{T}(\mathcal{C})$ is left adjoint to $\mathcal{U}_{T}$ and the adjunction $\left(\mathcal{F}_{T}, \mathcal{U}_{T}\right)$ is monadic with monad $Z_{T}$ (see Remark 3.1). If $T$ is a bimonad, this adjunction is comonoidal and $Z_{T}$ is its associated bimonad (see Theorem 3.3).

A monoidal category $\mathcal{C}$ is said to be centralizable if its identity endofunctor $1_{\mathcal{C}}$ is centralizable. In such a case, the centralizer of $1_{\mathcal{C}}$ is called the centralizer of $\mathcal{C}$. In view of Remark 5.10, we have:

Corollary 5.14. Let $\mathcal{C}$ be a centralizable autonomous category with centralizer $(Z, \partial)$. Then the forgetful functor $\mathcal{U}: \mathcal{Z}(\mathcal{C}) \rightarrow \mathcal{C}$ is monadic with monad $Z$. In fact $Z$ is a Hopf monad and the functor $Z-\mathcal{C} \rightarrow \mathcal{Z}(\mathcal{C})$, defined by

$$
(M, r) \mapsto\left(M,\left(\operatorname{id}_{1_{\mathcal{C}}} \otimes r\right) \partial_{M, 1_{\mathcal{C}}}\right) \quad \text { and } \quad f \mapsto f,
$$

is a strict monoidal isomorphism of categories.

Remark 5.15. The monadicity assertion of Corollary 5.14 is a consequence of DS07, Theorem 4.3].

Remark 5.16. We will see in Section 6.2 that $R=(u \otimes \mathrm{id}) \partial$ is an R-matrix for $Z$ (where $u$ denotes the unit of $Z$ ), making the isomorphism of Corollary 5.14 an isomorphism of braided categories.

5.7. Proof of Theorem 5.12. Throughout this section, let $T$ be a centralizable endofunctor of a left autonomous category $\mathcal{C}$, with centralizer $\left(Z_{T}, \partial\right)$. Recall that $Z_{T}$ is a monad by Theorem 5.6(a). Denote by $m$ and $u$ its product and unit.

Remark first that, by Lemma 5.4 for any object $M$ of $\mathcal{C}$, we have a bijection:

$$
\left\{\begin{array}{cl}
\operatorname{Hom}_{\mathcal{C}}\left(Z_{T}(M), M\right) & \rightarrow \operatorname{Hom}\left(M \otimes 1_{\mathcal{C}}, T \otimes M\right) \\
r & \mapsto \sigma_{(M, r)}=\left\{\left(\operatorname{id}_{T(Y)} \otimes r\right) \partial_{M, Y}\right\}_{Y \in \mathrm{Ob}(\mathcal{C})}
\end{array} .\right.
$$

Lemma 5.17. Let $M$ be an object of $\mathcal{C}$ and $r: Z_{T}(M) \rightarrow M$ be a morphism in $\mathcal{C}$. Then $(M, r)$ is a $Z_{T}$-module if and only if $\left(M, \sigma_{(M, r)}\right)$ is an object of $\mathcal{Z}_{T}(\mathcal{C})$.

Proof. By definition of the multiplication $m$ of $Z_{T}$, we have

$$
\left(T_{2} \otimes \mathrm{id}_{M}\right)\left(\sigma_{(M, r)}\right)_{\otimes}=\left(T_{2} \otimes r\right) \partial_{M, \otimes}=\left(\mathrm{id}_{T^{\otimes 2}} \otimes r m_{M}\right) \partial_{M, 1_{\mathcal{C}}, 1_{\mathcal{C}}}^{2} .
$$

Moreover,

$$
\left(\mathrm{id}_{T} \otimes \sigma_{(M, r)}\right)\left(\sigma_{(M, r)} \otimes \mathrm{id}_{1_{\mathcal{C}}}\right)=\left(\mathrm{id}_{T \otimes 2} \otimes r Z_{T}(r)\right) \partial_{M, 1_{\mathcal{C}}, 1_{\mathcal{C}}}^{2} .
$$

Therefore, by Lemma 5.4, $\left(T_{2} \otimes \mathrm{id}_{M}\right)\left(\sigma_{(M, r)}\right)_{\otimes}=\left(\operatorname{id}_{T} \otimes \sigma_{(M, r)}\right)\left(\sigma_{(M, r)} \otimes \mathrm{id}_{1_{\mathcal{C}}}\right)$ if and only if $r m_{M}=r Z_{T}(r)$. Also, since $\left(T_{0} \otimes \operatorname{id}_{M}\right)\left(\sigma_{(M, r)}\right)_{\mathbb{1}}=\left(T_{0} \otimes r\right) \partial_{M, \mathbb{1}}=r u_{M}$, we have $\left(T_{0} \otimes \operatorname{id}_{M}\right)\left(\sigma_{(M, r)}\right)_{\mathbb{1}}=\mathrm{id}_{M}$ if and only if $r u_{M}=\mathrm{id}_{M}$. 
Lemma 5.18. Let $(M, r)$ and $(N, s)$ be two $Z_{T}$-modules. Let $f: M \rightarrow N$ be a morphism in $\mathcal{C}$. Then $f$ is $Z_{T}$ - linear if and only if it is a morphism from $\left(M, \sigma_{(M, r)}\right)$ to $\left(N, \sigma_{(N, s)}\right)$ in $\mathcal{Z}_{T}(\mathcal{C})$.

Proof. We have $\left(\mathrm{id}_{T} \otimes f\right) \sigma_{(M, r)}=\left(\mathrm{id}_{T} \otimes f r\right) \partial_{M, 1_{\mathcal{C}}}$ and

$$
\sigma_{(N, s)}\left(f \otimes \mathrm{id}_{T}\right)=\left(\mathrm{id}_{T} \otimes s\right) \partial_{N, 1_{\mathcal{C}}}\left(f \otimes \mathrm{id}_{1_{\mathcal{C}}}\right)=\left(\operatorname{id}_{T} \otimes s Z_{T}(f)\right) \partial_{M, 1_{\mathcal{C}}} .
$$

Therefore, by Lemma 5.4, we obtain $\left(\mathrm{id}_{T} \otimes f\right) \sigma_{(M, r)}=\sigma_{(N, s)}\left(f \otimes \mathrm{id}_{T}\right)$ if and only if $f r=s Z_{T}(f)$.

Using Lemmas 5.17 and 5.18, one sees that the functor $E: Z_{T^{-}} \mathcal{C} \rightarrow \mathcal{Z}_{T}(\mathcal{C})$, given by $E(M, r)=\left(M, \sigma_{(M, r)}\right)$ and $E(f)=f$, is a well-defined isomorphism of categories. Furthermore it clearly satisfies $\mathcal{U}_{T} E=U_{Z_{T}}$.

Assume now that $(T, \mu, \eta)$ is a bimonad. Then $Z_{T}$ is a bimonad by Theorem $\left[5.6(\mathrm{c})\right.$ and the category $\mathcal{Z}_{T}(\mathcal{C})$ is monoidal by Proposition [5.9, Since, for any $Z_{T}$ - modules $(M, r)$ and $(N, s)$, we have

$$
\begin{aligned}
E(M, r) \otimes E(N, s) & =\left(M, \sigma_{(M, r)}\right) \otimes\left(N, \sigma_{(N, s)}\right) \\
& =\left(M \otimes N,(\mu \otimes r \otimes s)\left(\partial_{M, T} \otimes i_{Z_{T}(N)}\right)\left(i_{M} \otimes \partial_{N, 1_{\mathcal{C}}}\right)\right) \\
& =\left(M \otimes N,\left(i_{T} \otimes(r \otimes s)\left(Z_{T}\right)_{2}(M, N)\right) \partial_{M \otimes N, 1_{\mathcal{C}}}\right) \\
& =E((M, r) \otimes(N, s))
\end{aligned}
$$

and $E\left(\mathbb{1},\left(Z_{T}\right)_{0}\right)=\left(\mathbb{1},\left(\operatorname{id}_{T} \otimes\left(Z_{T}\right)_{0}\right) \partial_{\mathbb{1}, 1_{\mathcal{C}}}\right)=(\mathbb{1}, \eta)$, the functor $E$ is strict monoidal. Finally, we have $\mathcal{U}_{T} E=U_{Z_{T}}$ as monoidal functors because the forgetful functors $U_{Z_{T}}: Z_{T^{-}} \mathcal{C} \rightarrow \mathcal{C}$ and $\mathcal{U}_{T}: \mathcal{Z}_{T}(\mathcal{C}) \rightarrow \mathcal{C}$ are strict monoidal.

5.8. End of the proof of Theorem [5.6. Let us prove part (d) of Theorem 5.6. Let $(T, \mu, \eta)$ be a centralizable bimonad on an autonomous category $\mathcal{C}$, with centralizer $\left(Z_{T}, \partial\right)$. By Theorem 5.6(c), $Z_{T}$ is a bimonad. By Theorem 5.12, the functor $E: Z_{T^{-}} \mathcal{C} \rightarrow \mathcal{Z}_{T}(\mathcal{C})$, defined by

$$
E(M, r)=\left(M,\left(\operatorname{id}_{T} \otimes r\right) \partial_{M, 1_{\mathcal{C}}}\right) \text { and } E(f)=f,
$$

is a strict monoidal isomorphism. Assume $T$ admits a right antipode $s^{r}$. Then the category $\mathcal{Z}_{T}(\mathcal{C})$ is left autonomous by Proposition 5.9. Hence the category $Z_{T^{-}} \mathcal{C}$ is left autonomous, and so $Z_{T}$ admits a left antipode by Theorem 2.7(b). Denote by $m$ the product of $Z_{T}, u$ its unit, and $S^{l}$ its right antipode. Let $X$ be an object of $\mathcal{C}$. In the category $Z_{T^{-}} \mathcal{C}$, we have a duality

$$
\left({ }^{\vee}\left(Z_{T}(X), m_{X}\right),\left(Z_{T}(X), m_{X}\right), \operatorname{ev}_{Z_{T}(X)}, \operatorname{coev}_{Z_{T}(X)}\right),
$$

where ${ }^{\vee}\left(Z_{T}(X), m_{X}\right)=\left({ }^{\vee} Z_{T}(X), S_{Z_{T}(X)}^{l} Z_{T}\left({ }^{\vee} m_{T}\right)\right)$. Hence, $E$ being strict monoidal, we have a duality in the category $\mathcal{Z}_{T}(\mathcal{C})$ :

$$
\left(E\left({ }^{\vee}\left(Z_{T}(X), m_{X}\right)\right), E\left(Z_{T}(X), m_{X}\right), \operatorname{ev}_{Z_{T}(X)}, \operatorname{coev}_{Z_{T}(X)}\right),
$$

where $E\left({ }^{\vee}\left(Z_{T}(X), m_{X}\right)\right)=\left({ }^{\vee} Z_{T}(X),\left(\operatorname{id}_{T} \otimes S_{Z_{T}(X)}^{l} Z_{T}\left({ }^{\vee} m_{T}\right)\right) \partial^{\vee} Z_{T}(X), 1_{\mathcal{C}}\right)$. Now, by Proposition 5.9, we also have the following duality in $\mathcal{Z}_{T}(\mathcal{C})$ :

$$
\left({ }^{\vee} E\left(Z_{T}(X), m_{X}\right), E\left(Z_{T}(X), m_{X}\right), \mathrm{ev}_{Z_{T}(X)}, \operatorname{coev}_{Z_{T}(X)}\right),
$$

where ${ }^{\vee} E\left(Z_{T}(X), m_{X}\right)=\left({ }^{\vee} Z_{T}(X),{ }^{\vee}\left(\left(s^{r} \otimes m_{X}\right) \partial_{Z_{T}(X), T^{\vee}}\right)\right)$. Hence, by uniqueness of duals up to unique isomorphism,

$$
\left(\operatorname{id}_{T} \otimes S_{Z_{T}(X)}^{l} Z_{T}\left({ }^{\vee} m_{T}\right)\right) \partial^{\vee} Z_{T}(X), 1_{\mathcal{C}}={ }^{\vee}\left(\left(s^{r} \otimes m_{X}\right) \partial_{Z_{T}(X), T^{\vee}}\right) .
$$


Composing on the left with $\left(\operatorname{id}_{T} \otimes{ }^{\vee} u_{X}\right)={ }^{\vee}\left(u_{X} \otimes \operatorname{id}_{T^{\vee}}\right)$, we get

$$
\left(\mathrm{id}_{T} \otimes S_{X}^{l}\right) \partial{ }^{{ }} Z_{T}(X), 1_{\mathcal{C}}={ }^{\vee}\left(\left(s^{r} \otimes \operatorname{id}_{Z_{T}(X)}\right) \partial_{X, T^{\vee}}\right),
$$

which is the defining relation of Theorem 5.6(d). Hence we have proved part (d) of Theorem 5.6. Part (e) can be shown similarly.

\section{The double of A Hopf Monad}

Given a centralizable Hopf monad $T$ on an autonomous category $\mathcal{C}$, we construct the canonical distributive law $\Omega$ of $T$ over its centralizer $Z_{T}$, which serves two purposes. First, $\Omega$ gives rise to a new Hopf $\operatorname{monad} D_{T}=Z_{T} \circ_{\Omega} T$, called the double of $T$. The double $D_{T}$ is actually quasitriangular and $Z(T-\mathcal{C}) \simeq D_{T^{-}} \mathcal{C}$ as braided categories, see Section 6.2. Second, $\Omega$ defines a lift of the Hopf monad $Z_{T}$ to a Hopf monad $\tilde{Z}_{T}^{\Omega}$ on $T$ - $\mathcal{C}$, which turns out to be the centralizer of the category $T$ - $\mathcal{C}$, and so $\tilde{Z}_{T}^{\Omega}\left(\mathbb{1}, T_{0}\right)$ is the coend of $T$ - $\mathcal{C}$, see Section 6.3 .

Most of the results of this section are special cases of results of Section 7 . We state them here for convenience.

6.1. The canonical distributive law. Let $T$ be a centralizable Hopf monad on an autonomous category $\mathcal{C}$ and $\left(Z_{T}, \partial\right)$ be its centralizer.

Recall (see Proposition [5.3) that $Z_{T}(X)=\int{ }^{Y \in \mathcal{C} \vee} T(Y) \otimes X \otimes Y$, with universal dinatural transformation

$$
i_{X, Y}=\left(\operatorname{coev}_{T(Y)} \otimes \operatorname{id}_{Z_{T}(X)}\right)\left(\operatorname{idv}_{T(X)} \otimes \partial_{X, Y}\right),
$$

which is natural in $X$ and dinatural in $Y$. Since $T(i)$ is a universal dinatural transformation (see Section 3.5), we can define a natural transformation $\Omega: T Z_{T} \rightarrow Z_{T} T$ by

$$
\Omega_{X} T\left(i_{X, Y}\right)=i_{T(X), T(Y)}\left({ }^{\vee} \mu_{Y} s_{T(Y)}^{l} T\left({ }^{\vee} \mu_{Y}\right) \otimes \operatorname{id}_{T(X) \otimes T(Y)}\right) T_{3}\left({ }^{\vee} T(Y), X, Y\right),
$$

where $\mu$ and $s^{l}$ are the product and left antipode of $T$ and where $T_{3}: T \otimes_{3} \rightarrow T^{\otimes 3}$ is defined as in Section 1.5 .

Theorem 6.1. The natural transformation $\Omega: T Z_{T} \rightarrow Z_{T} T$ is an invertible comonoidal distributive law.

We call $\Omega$ the canonical distributive law of $T$. We prove Theorem 6.1 in Section 7.4 .

The inverse $\Omega^{-1}: Z_{T} T \rightarrow T Z_{T}$ of the distributive law $\Omega$ is the natural transformation defined by

$$
\begin{aligned}
& \Omega_{X}^{-1} i_{T(X), Y}=\left(\operatorname{ev}_{T(Y)}\left(\operatorname{idv}_{T(Y)} \otimes \mu_{Y} T\left(\mu_{Y}\right)\right) \otimes T\left(i_{X, T(Y)}\right) \otimes \operatorname{ev}_{Y}\left(s_{Y}^{l} \otimes \operatorname{id}_{Y}\right)\right) \\
& \quad \circ T_{3}\left(T^{2}(Y),{ }^{\vee} T^{2}(Y) \otimes X \otimes T(Y),{ }^{\vee} T(Y)\right) T\left(\operatorname{coev}_{T^{2}(Y)} \otimes \operatorname{id}_{X} \otimes \operatorname{coev}_{T(Y)}\right) .
\end{aligned}
$$

Remark 6.2. The canonical distributive law of $T$ is the only natural transformation $\Omega: T Z_{T} \rightarrow Z_{T} T$ satisfying

$$
(\mu \otimes \Omega) T_{2} T(\partial)=\left(\mu \otimes \operatorname{id}_{Z_{T} T}\right) \partial_{T, T} T_{2} .
$$

Remark 6.3. One can show that R-matrices for $T$ correspond bijectively with morphisms of Hopf monads $f: Z_{T} \rightarrow T$ satisfying $\mu T(f)=\mu f_{T} \Omega$. The R-matrix associated with such a morphism $f$ is $R=\left(\operatorname{id}_{T} \otimes f\right) \partial$. 
6.2. The double of a Hopf monad. Let $T$ be a centralizable Hopf monad on an autonomous category $\mathcal{C}$, with centralizer $\left(Z_{T}, \partial\right)$. Let $\Omega: T Z_{T} \rightarrow Z_{T} T$ be the canonical distributive law of $T$. By Corollary 4.11 .

$$
D_{T}=Z_{T} \circ_{\Omega} T
$$

is a Hopf monad on $\mathcal{C}$. Denote by $\eta$ and $u$ the units of $T$ and $Z_{T}$, respectively.

Theorem 6.4. The natural transformation $R=\left\{R_{X, Y}\right\}_{X, Y \in \mathrm{Ob}(\mathcal{C})}$, defined by

$$
R_{X, Y}=\left(u_{T(Y)} \otimes Z_{T}\left(\eta_{X}\right)\right) \partial_{X, Y}: X \otimes Y \rightarrow D_{T}(Y) \otimes D_{T}(X),
$$

is an R-matrix for the Hopf monad $D_{T}$.

The quasitriangular Hopf monad $D_{T}$ is called the double of $T$. This terminology is justified by the fact that the braided categories $Z(T-\mathcal{C})$ and $D_{T^{-}} \mathcal{C}$ coincide. More precisely, let $\mathcal{U}: \mathcal{Z}(T-\mathcal{C}) \rightarrow \mathcal{C}$ be the strict monoidal forgetful functor defined by

$$
\mathcal{U}((M, r), \sigma)=M \quad \text { and } \quad \mathcal{U}(f)=f .
$$

Let $I: D_{T^{-}} \mathcal{C} \rightarrow \mathcal{Z}(T-\mathcal{C})$ be the functor defined by $I(f)=f$ and

$$
I(M, r)=\left(\left(M, r u_{T(M)}\right), \sigma\right) \quad \text { with } \quad \sigma_{(N, s)}=\left(s \otimes r Z_{T}\left(\eta_{M}\right)\right) \partial_{M, N} .
$$

Theorem 6.5. The functor I is a strict monoidal isomorphism of braided categories such that the following triangle of monoidal functors commutes:

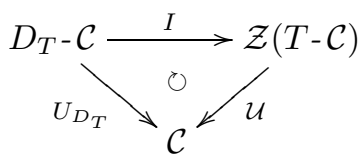

We prove Theorems 6.4 and 6.5 in Section 7.5

Remark 6.6. The functor $\mathcal{F}=I F_{D_{T}}: \mathcal{C} \rightarrow \mathcal{Z}(T-\mathcal{C})$ is left adjoint to $\mathcal{U}$, and the adjunction $(\mathcal{F}, \mathcal{U})$ is monadic with monad $D_{T}$ (see Remark 3.1). Moreover, $D_{T}$ is the Hopf monad associated with this comonoidal adjunction (see Theorem 3.3).

Remark 6.7. According to Remark 5.1 the construction of the double of a Hopf monad $T$ admits a 'right-handed' version: if $Z_{T}^{\prime}$ is a 'right-handed' centralizer of $T$, there exists a 'right-handed' canonical law $\Omega^{\prime}$ of $T$ over $Z_{T}^{\prime}$, and hence a Hopf monad $D_{T}^{\prime}=Z_{T}^{\prime}{ }^{\circ} \Omega^{\prime} T$ endowed with an R-matrix $R^{\prime}$ such that $D_{T^{-}}^{\prime} \mathcal{C} \simeq \mathcal{Z}^{\prime}(T-\mathcal{C})$ as braided categories. If we identify $Z_{T}^{\prime}$ to $Z_{T}$ as in Remark [5.8, then $\Omega^{\prime}=\Omega$, $D_{T}^{\prime}=D_{T}$ as Hopf monads, and $R^{\prime}=R^{*-1}$.

Remark 6.8. Let $T$ be a centralizable Hopf monad on an autonomous category $\mathcal{C}$ and $\left(Z_{T}, \partial\right)$ be its centralizer. Denote by $\eta$ and $u$ the units of $T$ and $Z_{T}$, respectively. Assuming $u_{T}: T \rightarrow Z_{T} T$ is a monomorphism, one can show that the canonical distributive law of $T$ is the only comonoidal distributive law $\Omega: T Z_{T} \rightarrow Z_{T} T$ such that

$$
R=\left(u_{T} \otimes Z_{T}(\eta)\right) \partial
$$

is an R-matrix for the Hopf monad $Z_{T} \circ_{\Omega} T$. This generalizes Drinfeld's original characterization of the double of a finite-dimensional Hopf algebra. 
6.3. The centralizer and the coend of a category of modules. Let $T$ be a centralizable Hopf monad on an autonomous category $\mathcal{C}$. Let $\left(Z_{T}, \partial\right)$ be the centralizer of $T$ and $\Omega: T Z_{T} \rightarrow Z_{T} T$ be the canonical distributive law of $T$. By Corollary 4.11, $\tilde{Z}_{T}^{\Omega}$ is Hopf monad which is a lift of the Hopf monad $Z_{T}$ to $T$ - $\mathcal{C}$. Recall that

$$
\tilde{Z}_{T}^{\Omega}(M, r)=\left(Z_{T}(M), Z_{T}(r) \Omega_{M}\right) \quad \text { and } \quad \tilde{Z}_{T}^{\Omega}(f)=Z_{T}(f)
$$

For any $T$-modules $(M, r)$ and $(N, s)$, set

$$
\tilde{\partial}_{(M, r),(N, s)}=\left(s \otimes \mathrm{id}_{Z_{T}(M)}\right) \partial_{M, N}:(M, r) \otimes(N, s) \rightarrow(N, s) \otimes \tilde{Z}_{T}^{\Omega}(M, r) .
$$

Theorem 6.9. The pair $\left(\tilde{Z}_{T}^{\Omega}, \tilde{\partial}\right)$ is a centralizer of the category $T-\mathcal{C}$.

We prove Theorem 6.9 in Section 7.6 .

Recall that

$$
Z_{T}(\mathbb{1})=\int^{Y \in \mathcal{C}} v_{T}(Y) \otimes Y
$$

with universal dinatural transformation $i_{Y}=\left(\operatorname{ev}_{T(Y)} \otimes \mathrm{id}_{Z_{T}(\mathbb{1})}\right) \partial_{\mathbb{1}, Y}$. Denote by $\alpha=Z_{T}\left(T_{0}\right) \Omega_{\mathbb{1}}$ the $T$-action of $\tilde{Z}_{T}^{\Omega}\left(\mathbb{1}, T_{0}\right)$. It is characterized by

$$
\alpha T\left(i_{Y}\right)=i_{T(Y)}\left({ }^{\vee} \mu_{Y} s_{T(Y)}^{l} T\left({ }^{\vee} \mu_{Y}\right) \otimes \operatorname{id}_{T(Y)}\right) T_{2}\left({ }^{\vee} T(Y), Y\right)
$$

By Theorem 6.9 and Proposition $5.3, \tilde{Z}_{T}^{\Omega}\left(\mathbb{1}, T_{0}\right)=\left(Z_{T}(\mathbb{1}), \alpha\right)$ is the coend of $T-\mathcal{C}$. That is,

$$
\left(Z_{T}(\mathbb{1}), \alpha\right)=\int^{(M, r) \in T-\mathcal{C}} \vee(M, r) \otimes(M, r),
$$

with universal dinatural transformation $\tilde{\imath}_{(M, r)}=i_{M}\left({ }^{\vee} r \otimes M\right)$.

The coend $\left(Z_{T}(\mathbb{1}), \alpha\right)$ of $T-\mathcal{C}$ is a coalgebra in $T-\mathcal{C}$, with coproduct and counit given by

$$
\Delta=\left(Z_{T}\right)_{2}(\mathbb{1}, \mathbb{1}): Z_{T}(\mathbb{1}) \rightarrow Z_{T}(\mathbb{1}) \otimes Z_{T}(\mathbb{1}) \quad \text { and } \quad \varepsilon=\left(Z_{T}\right)_{0}: Z_{T}(\mathbb{1}) \rightarrow \mathbb{1}
$$

Assume now that $T$ is furthermore quasitriangular, with $\mathrm{R}$-matrix $R$, so that the autonomous category $T-\mathcal{C}$ is braided. Then the coalgebra $\left(\left(Z_{T}(\mathbb{1}), \alpha\right), \Delta, \varepsilon\right)$ becomes a Hopf algebra in $T-\mathcal{C}$ endowed with a self-dual Hopf pairing (see Section 8.3). Its unit is

$$
u=\left(T_{0} \otimes \operatorname{id}_{Z_{T}(\mathbb{1})}\right) \partial_{\mathbb{1}, \mathbb{1}}: \mathbb{1} \rightarrow Z_{T}(\mathbb{1}) .
$$

Its product $m$, antipode $S$, and Hopf pairing $\omega$ are given in Figure 2 .

Remark 6.10. In Sectgion 9.3, we treat the case of the centralizer of a fusion category $\mathcal{F}$ (which is a quasitriangular Hopf monad by Theorem 6.5) to get a convenient description of the coend of $\mathcal{Z}(\mathcal{F})$. 


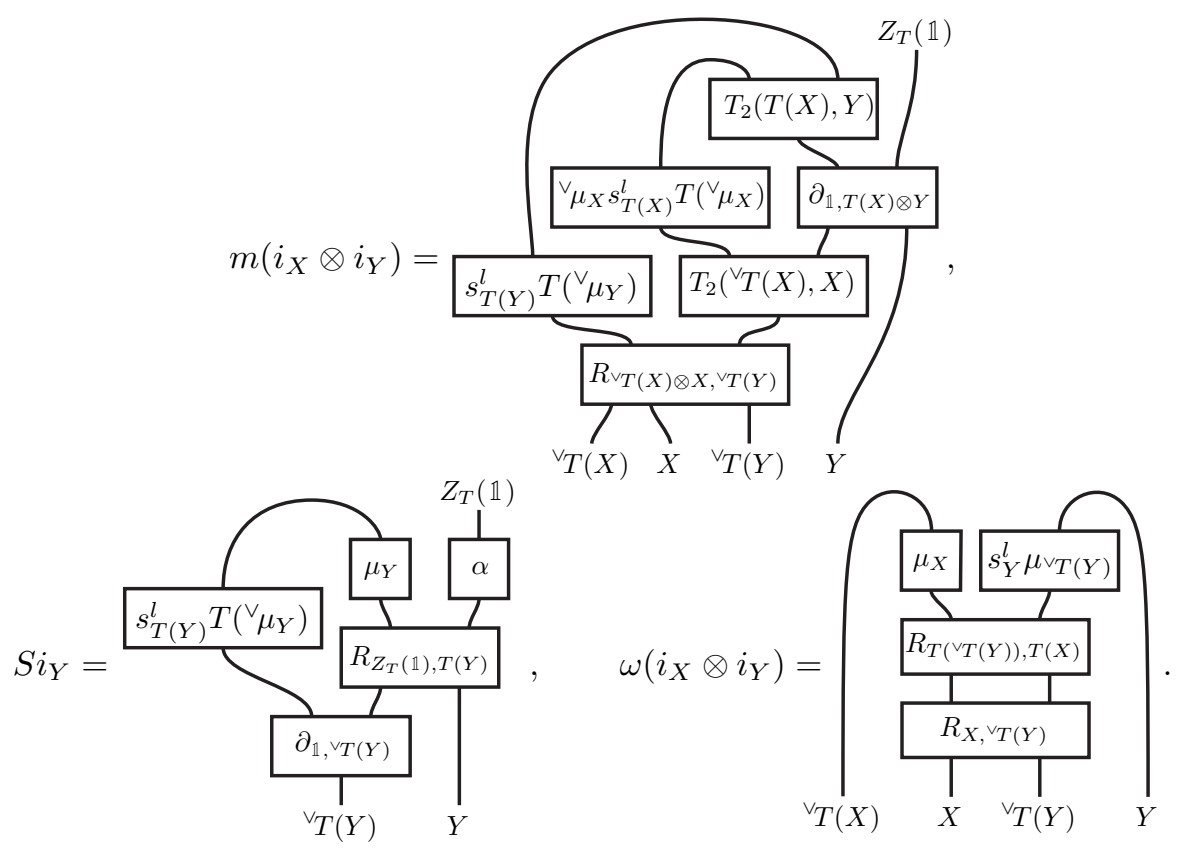

FiguRE 2. Hopf algebra structure of the coend of $T$ - $\mathcal{C}$

\section{The Centralizer of a Hopf monad on a CATEgory of MOdules}

In this section, we study the centralizer of a Hopf monad $Q$ on the category $T$ - $\mathcal{C}$ of modules over a Hopf monad $T$ on an autonomous category $\mathcal{C}$. We show that it is centralizable whenever the cross product $Q \rtimes T$ is centralizable. In that case, the centralizer of $Q \rtimes T$ lifts naturally to a centralizer of $Q$, which also turns out to be a lift of Hopf monads. Hence we have a canonical distributive law $\Omega$ of $T$ over $Z_{Q \rtimes T}$ and a Hopf monad $D_{Q, T}=Z_{Q \rtimes T} \circ_{\Omega} T$ on $\mathcal{C}$. We interpret the category of $D_{Q, T}$ modules as the center of $T-\mathcal{C}$ relative to $Q$.

7.1. Centralizability on categories of modules. In this section, given a Hopf monad $T$ on an autonomous category $\mathcal{C}$, we give a criterion for an endofunctor $Q$ of $T$ - $\mathcal{C}$ to be centralizable in terms of the centralizability of the cross product $Q \rtimes T$ on $\mathcal{C}$ (see Section 3.7 for the definition of cross products).

Proposition 7.1. Let $T$ be a Hopf monad on an autonomous category $\mathcal{C}$ and let $Q$ be an endofunctor of $T$ - $\mathcal{C}$. Let $(M, r)$ be a $T$-module. Then:

(a) The endofunctor $Q$ is centralizable at $(M, r)$ if and only if $Q \rtimes T$ is centralizable at $U_{T}(M, r)=M$.

(b) Assume $Q \rtimes T$ is centralizable at $M$, with centralizer $(Z, \delta)$. Then $Q$ admits a unique centralizer $(\tilde{Z}, \tilde{\delta})$ at $(M, r)$ such that

$$
U_{T}(\tilde{Z})=Z \quad \text { and } \quad \tilde{\delta}_{(N, s)}=\left(Q(s) \otimes \operatorname{id}_{Z}\right) \delta_{N}
$$

for any $T$-module $(N, s)$. 
Remark 7.2. In the second formula of Proposition 7.1(b), $Q(s)$ makes sense because $s:\left(T(N), \mu_{N}\right) \rightarrow(N, s)$ is a morphism in $T$ - $\mathcal{C}$. This formula can be written as

$$
\tilde{\delta}=\left(Q(\varepsilon) \otimes \operatorname{id}_{Z}\right) \delta,
$$

where $\varepsilon$ denotes the counit of the adjunction $\left(U_{T}, F_{T}\right)$.

Proof of Proposition 7.1. Let us prove part (a). Fix a $T$-module $(M, r)$. By Proposition 5.3 $Q$ is centralizable at $(M, r)$ if and only if the coend

$$
\int^{(N, s) \in T-\mathcal{C}}{ }^{\vee} Q(N, s) \otimes(M, r) \otimes(N, s)
$$

exists. Since the functor $U_{T}$ creates and preserves coends (see Section 3.5) and is strict monoidal, this is equivalent to the existence of the coend

$$
\begin{gathered}
\int^{(N, s) \in T-\mathcal{C}} U_{T}\left({ }^{\vee} Q(N, s) \otimes(M, r) \otimes(N, s)\right) \\
=\int^{(N, s) \in T-\mathcal{C}}{ }^{{ }^{(} U_{T} Q(N, s) \otimes M \otimes U_{T}(N, s) .}
\end{gathered}
$$

By Lemma 3.9, this is equivalent to the existence of the coend

$$
\int^{Y \in \mathcal{C}}{ }^{\vee} U_{T} Q F_{T}(Y) \otimes M \otimes Y=\int^{Y \in \mathcal{C}}{ }^{\vee} Q \rtimes T(Y) \otimes M \otimes Y,
$$

and so, by Proposition 5.3. to the fact that $Q \rtimes T$ is centralizable at $M$.

Let us prove part (b). By Proposition 5.3, we have

$$
Z=\int^{Y \in \mathcal{C}}{ }^{\vee} Q \rtimes T(Y) \otimes M \otimes Y,
$$

with universal dinatural transformation $i_{Y}=\left(\operatorname{ev}_{Q \rtimes T(Y)} \otimes \operatorname{id}_{Z}\right)\left(\operatorname{id}_{Q} v_{\rtimes T(Y)} \otimes \delta_{Y}\right)$. By Lemma 3.9, we also have

$$
Z=\int^{(N, s) \in T-\mathcal{C}} \vee U_{T} Q(N, s) \otimes M \otimes U_{T}(N, s)
$$

with universal dinatural transformation $j_{(N, s)}=i_{N}\left({ }^{\vee} U_{T} Q(s) \otimes \operatorname{id}_{M \otimes N}\right)$. Set

$$
\tilde{\delta}_{(N, s)}=\left(\operatorname{id}_{Q(N, s)} \otimes j_{(N, s)}\right)\left(\operatorname{coev}_{Q(N, s)} \otimes \operatorname{id}_{M}\right) .
$$

By Proposition 3.10, there exists a unique $T$-action $\alpha: T(Z) \rightarrow Z$ such that $j_{(N, s)}$, or equivalently $\tilde{\delta}_{(N, s)}$, is $T$ - linear for all $T$-modules $(N, s)$. Furthermore we have

$$
(Z, \alpha)=\int^{(N, s) \in T-\mathcal{C}} \vee Q(N, s) \otimes(M, r) \otimes(N, s)
$$

with universal dinatural transformation $j$. Set $\tilde{Z}=(Z, \alpha)$. By Proposition [5.3, $(\tilde{Z}, \tilde{\delta})$ is a centralizer of $Q$ at $(M, r)$. By construction, we have $U_{T}(\tilde{Z})=Z$ and $\tilde{\delta}_{(N, s)}=\left(U_{T} Q(s) \otimes \operatorname{id}_{Z}\right) \delta_{N}$ for every $T$-module $(N, s)$. Furthermore, since $\alpha$ is the only action of $T$ on $Z=U_{T}(\tilde{Z})$ such that every $\tilde{\delta}_{(N, s)}$ is $T$-linear, $(\tilde{Z}, \tilde{\delta})$ is the only centralizer of $Q$ at $(M, r)$ satisfying the conditions of part (b).

Applying Lemma 5.2 and Proposition 7.1(a), we immediately deduce:

Corollary 7.3. Let $T$ be a Hopf monad on an autonomous category $\mathcal{C}$ and let $Q$ be an endofunctor of $T-\mathcal{C}$. Then $Q$ is centralizable if and only if, for any $T$-module $(M, r)$, the endofunctor $Q \rtimes T$ of $\mathcal{C}$ is centralizable at $M$. 
7.2. Lifting centralizers. In this section, given a centralizable Hopf monad $T$ on an autonomous category $\mathcal{C}$ and an endofunctor $Q$ of $T-\mathcal{C}$, we show that a centralizer of $Q \rtimes T$ lifts uniquely to a centralizer of $Q$. Furthermore, if $Q$ is comonoidal (resp. a bimonad), then it is also a lift as a monad (resp. a bimonad).

Theorem 7.4. Let $T$ be a Hopf monad on an autonomous category $\mathcal{C}$ and let $Q$ be an endofunctor of $T$ - $\mathcal{C}$. Assume $Q \rtimes T$ is centralizable, with centralizer $\left(Z_{Q \rtimes T}, \partial\right)$. Then:

(a) The centralizer of $Q \rtimes T$ lifts uniquely to a centralizer of $Q$. More precisely, $Q$ admits a unique centralizer $\left(Z_{Q}, \tilde{\partial}\right)$ such that $U_{T} Z_{Q}=Z_{Q \rtimes T} U_{T}$ and

$$
\tilde{\partial}_{(M, r),(N, s)}=\left(Q(s) \otimes \operatorname{id}_{Z_{Q \rtimes T}(M)}\right) \partial_{M, N}
$$

for all $T$-modules $(M, r)$ and $(N, s)$.

(b) If $Q$ is comonoidal, the monad $Z_{Q}$ is a lift of the monad $Z_{Q \rtimes T}$ to T-C.

(c) If $Q$ is a bimonad, the bimonad $Z_{Q}$ is a lift of the bimonad $Z_{Q \rtimes T}$ to T-C.

Proof. Part (a) is a direct consequence of Lemma 5.2 and Proposition 7.1(b). Let $\left(Z_{Q}, \tilde{\partial}\right)$ be the centralizer of $Q$ given by part (a).

Assume $Q$ is comonoidal. Then $Q \rtimes T$ is comonoidal by Section 3.7. Therefore both $Z_{Q}$ and $Z_{Q \rtimes T}$ are monads by Theorem 5.6(a). Denote by $\eta$ and $\varepsilon$ the unit and counit of the adjunction $\left(U_{T}, F_{T}\right)$. By part (a), we have

$$
U_{T}(\tilde{\partial})=\left(U_{T} Q(\varepsilon) \otimes \operatorname{id}_{Z_{Q \rtimes T} U_{T}}\right) \partial_{U_{T}, U_{T}} .
$$

By definition of the product $\tilde{m}$ of $Z_{Q}$, we have

$$
\left(\operatorname{id}_{Q F_{T} \otimes Q F_{T}} \otimes \tilde{m}\right) \tilde{\partial}_{1_{T-\mathcal{c}}, F_{T}, F_{T}}^{2}=\left(Q_{2}\left(F_{T}, F_{T}\right) \otimes \mathrm{id}_{Z_{Q}}\right) \tilde{\partial}_{1_{T-\mathcal{c}}, F_{T} \otimes F_{T}} .
$$

Hence, we get

$$
\begin{aligned}
\left(U_{T} Q\left(\varepsilon_{F_{T}}\right)\right. & \left.\otimes U_{T} Q\left(\varepsilon_{F_{T}}\right) \otimes U_{T}(\tilde{m})\right) \partial_{U_{T}, T, T}^{2} \\
& =\left(U_{T}\left(Q_{2}\left(F_{T}, F_{T}\right) Q\left(\varepsilon_{F_{T} \otimes F_{T}}\right)\right) \otimes i_{U_{T} Z_{Q}}\right) \partial_{U_{T}, T \otimes T} .
\end{aligned}
$$

Composing on the right with $\left(\mathrm{id}_{U_{T}} \otimes \eta \otimes \eta\right)$ and then using the expression of the comonoidal structure of $Q \rtimes T$ (see Section 3.7) and the identity $\varepsilon_{F_{T}} F_{T}(\eta)=\operatorname{id}_{F_{T}}$, we obtain

$$
\left(\operatorname{id}_{(Q \rtimes T)^{\otimes 2}} \otimes U_{T}(\tilde{m})\right) \partial_{U_{T}, 1_{\mathcal{C}}, 1_{\mathcal{C}}}^{2}=\left((Q \rtimes T)_{2} \otimes \operatorname{id}_{Z_{Q \rtimes T} U_{T}}\right) \partial_{U_{T}, \otimes},
$$

and so, by definition of the product $m$ of $Z_{Q \rtimes T}$, we have $U_{T}(\tilde{m})=m_{U_{T}}$. Moreover, denoting by $\tilde{u}$ and $u$ the units of $Z_{Q}$ and $Z_{Q \rtimes T}$, respectively, we have

$$
\begin{aligned}
U_{T}(\tilde{u}) & =U_{T}\left(\left(Q_{0} \otimes \operatorname{id}_{Z_{Q}}\right) \tilde{\partial}_{1_{T-\mathcal{c}},\left(\mathbb{1}, T_{0}\right)}\right) \\
& =\left(U_{T}\left(Q_{0}\right) U_{T} Q\left(T_{0}\right) \otimes \operatorname{id}_{U_{T} Z_{Q}}\right) \partial_{U_{T}, \mathbb{1}} \\
& =\left((Q \rtimes T)_{0} \otimes \operatorname{id}_{Z_{Q \rtimes T} U_{T}}\right) \partial_{U_{T}, \mathbb{1}}=u_{U_{T}} .
\end{aligned}
$$

Hence we have proved part (b).

Suppose now $Q$ is a bimonad. Then $Q \rtimes T$ is a bimonad (see Section 3.7). Therefore both $Z_{Q}$ and $Z_{Q \rtimes T}$ are bimonads by Theorem 5.6)(c). By definition of the morphism $\left(Z_{Q}\right)_{2}$, we have

$$
\left(\operatorname{id}_{Q F_{T}} \otimes\left(Z_{Q}\right)_{2}\right) \tilde{\partial}_{\otimes, F_{T}}=\left(q_{F_{T}} \otimes \operatorname{id}_{Z_{Q}^{\otimes 2}}\right)\left(\tilde{\partial}_{1_{T-\mathcal{c}}, Q F_{T}} \otimes \operatorname{id}_{Z_{Q}}\right)\left(\operatorname{id}_{1_{T-\mathcal{c}}} \otimes \tilde{\partial}_{1_{T-\mathcal{c}}, F_{T}}\right),
$$


where $q$ is the product of $Q$. Thus

$$
\begin{aligned}
\left(U_{T} Q\left(\varepsilon_{F_{T}}\right) \otimes U_{T}\left(\left(Z_{Q}\right)_{2}\right)\right) \partial_{U_{T} \otimes U_{T}, T}=\left(U_{T}\left(q_{F_{T}} Q\left(\varepsilon_{Q F_{T}}\right)\right) \otimes \operatorname{id}_{\left(U_{T} Z_{Q}\right)^{\otimes 2}}\right) & \\
& \circ\left(\partial_{U_{T}, Q \rtimes T} \otimes \operatorname{id}_{U_{T} Z_{Q}}\right)\left(\operatorname{id}_{U_{T}} \otimes\left(U_{T} Q\left(\varepsilon_{F_{T}}\right) \otimes \operatorname{id}_{U_{T} Z_{Q}}\right) \partial_{U_{T}, T}\right) .
\end{aligned}
$$

Composing on the right with $\left(\operatorname{id}_{U_{T}} \otimes \operatorname{id}_{U_{T}} \otimes \eta\right)$, since the product of $Q \rtimes T$ is given by $p=U_{T}\left(q_{F_{T}} Q\left(\varepsilon_{Q F_{T}}\right)\right)$, we obtain

$$
\begin{aligned}
\left(\operatorname{id}_{Q \rtimes T}\right. & \left.\otimes U_{T}\left(\left(Z_{Q}\right)_{2}\right)\right) \partial_{U_{T} \otimes U_{T}, 1_{\mathcal{C}}} \\
& =\left(p \otimes \operatorname{id}_{\left(Z_{Q \rtimes T} U_{T}\right)^{\otimes 2}}\right)\left(\partial_{U_{T}, Q \rtimes T} \otimes \operatorname{id}_{Z_{Q \rtimes T} U_{T}}\right)\left(\operatorname{id}_{U_{T}} \otimes \partial_{U_{T}, 1_{\mathcal{C}}}\right),
\end{aligned}
$$

and so, by definition of the morphism $\left(Z_{Q \rtimes T}\right)_{2}$, we obtain $\left(U_{T} Z_{Q}\right)_{2}=\left(Z_{Q \rtimes T} U_{T}\right)_{2}$. Now, by definition of the morphism $\left(Z_{Q}\right)_{0}$, we have

$$
\left(\operatorname{id}_{Q F_{T}} \otimes\left(Z_{Q}\right)_{0}\right) \tilde{\partial}_{\left(\mathbb{1}, T_{0}\right), F_{T}}=v_{F_{T}} .
$$

where $v$ is the unit of $Q$. Applying $U_{T}$ and composing with $\eta$, we get

$$
\left(\operatorname{id}_{Q \rtimes T} \otimes U_{T}\left(\left(Z_{Q}\right)_{0}\right)\right) \partial_{\mathbb{1}, 1_{\mathcal{C}}}=U_{T}\left(v_{F_{T}}\right) \eta .
$$

Since $U_{T}\left(v_{F_{T}}\right) \eta$ is the unit of $Q \rtimes T$ and by definition of the morphism $\left(Z_{Q \rtimes T}\right)_{0}$, we have $\left(U_{T} Z_{Q}\right)_{0}=\left(Z_{Q \rtimes T} U_{T}\right)_{0}$. Hence $U_{T} Z_{Q}=Z_{Q \rtimes T} U_{T}$ as comonoidal functors, and we have proved part $(\mathrm{c})$.

7.3. The canonical distributive law and the double. Throughout this section, let $T$ be a Hopf monad on an autonomous category $\mathcal{C}$ and $Q$ be a comonoidal endofunctor of $T$ - $\mathcal{C}$ such that $Q \rtimes T$ is centralizable with centralizer $\left(Z_{Q \rtimes T}, \partial\right)$.

By Theorem 7.4 the centralizer $\left(Z_{Q \rtimes T}, \partial\right)$ lifts to a centralizer $\left(Z_{Q}, \tilde{\partial}\right)$ of $Q$ and the monad $Z_{Q}$ is a lift of the monad $Z_{Q \rtimes T}$ to $T$ - $\mathcal{C}$. The monad

$$
D_{Q, T}=Z_{Q} \rtimes T
$$

is called the double of the pair $(Q, T)$. Since lifts correspond bijectively with distributive laws (see Theorem 4.5), there exists a unique distributive law $\Omega$ of $T$ over $Z_{Q \rtimes T}$ such that

$$
Z_{Q}=\tilde{Z}_{Q \rtimes T}^{\Omega}
$$

This distributive law is called the canonical distributive law of the pair $(Q, T)$. It provides a description of structure of the monad $D_{Q, T}$ :

$$
D_{Q, T}=Z_{Q \rtimes T} \circ_{\Omega} T \text {. }
$$

Proposition 7.5. (a) If $Q$ is a bimonad, then the canonical distributive law $\Omega$ is comonoidal, $D_{Q, T}$ is a bimonad, and $Z_{Q}=\tilde{Z}_{Q \rtimes T}^{\Omega}$ as bimonads.

(b) If $Q$ is a Hopf monad, then $D_{Q, T}$ is a Hopf monad.

Proof. Let us prove part (a). By Theorem 7.4, $Z_{Q}$ is a lift of $Z_{Q \rtimes T}$ as a bimonad. Therefore, by Theorem 4.7 $\Omega$ is comonoidal and $D_{Q, T}$ is a bimonad.

Let us prove part (b). Since $Q \rtimes T$ is a Hopf monad (see Section 3.7), so is $Z_{Q \rtimes T}$ (by Theorem 5.6). Therefore $D_{Q, T}$ is a Hopf monad (by Corollary 4.11).

Let $\mathcal{U}: \mathcal{Z}_{Q}(T-\mathcal{C}) \rightarrow \mathcal{C}$ be the functor defined as the composition of the forgetful functors $\mathcal{U}_{Q}: \mathcal{Z}_{Q}(T-\mathcal{C}) \rightarrow T-\mathcal{C}$ and $U_{T}: T-\mathcal{C} \rightarrow \mathcal{C}$, that is,

$$
\mathcal{U}((M, r), \sigma)=M \quad \text { and } \quad \mathcal{U}(f)=f .
$$


Denoting $\eta$ and $u$ the units of $T$ and $Z_{Q \rtimes T}$, let $I: D_{Q, T^{-}} \mathcal{C} \rightarrow \mathcal{Z}_{Q}(T-\mathcal{C})$ be the functor defined by

$$
I(M, r)=\left(\left(M, r u_{T(M)}\right), \sigma\right) \quad \text { and } \quad I(f)=f
$$

where $\sigma_{(N, s)}=\left(U_{T} Q(s) \otimes r Z_{Q \rtimes T}\left(\eta_{M}\right)\right) \partial_{M, N}$.

Theorem 7.6. The functor $I$ is an isomorphism of categories such that the following triangle commutes:

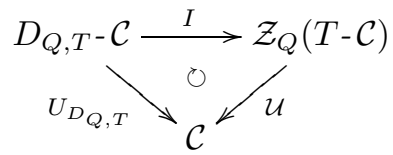

Furthermore, if $Q$ is a bimonad (so that $D_{Q, T}$ is a bimonad and $\mathcal{Z}_{Q}(T-\mathcal{C}$ ) is monoidal), then the functor $I$ is strict monoidal (and so $\mathcal{U} I=U_{D_{Q, T}}$ as monoidal functors).

Remark 7.7. The functor $\mathcal{F}=I F_{D_{Q}, T}: \mathcal{C} \rightarrow \mathcal{Z}_{Q}(T-\mathcal{C})$ is left adjoint to $\mathcal{U}$ and the adjunction $(\mathcal{F}, \mathcal{U})$ is monadic with monad $D_{Q, T}$ (see Remark 3.1). If $Q$ is a bimonad, this adjunction is comonoidal and $D_{Q, T}$ is its associated bimonad (see Theorem 3.3).

Proof. By Section 4.3, since $D_{Q, T}=Z_{Q \rtimes T} \circ_{\Omega} T$ and $Z_{Q}=\tilde{Z}_{Q \rtimes T}^{\Omega}$, the functor

$$
L:\left\{\begin{aligned}
D_{Q, T^{-}} \mathcal{C} & \longrightarrow Z_{Q^{-}}(T-\mathcal{C}) \\
(M, r) & \longmapsto\left(\left(M, r u_{T(M)}\right), r Z_{Q \rtimes T}\left(\eta_{M}\right)\right)
\end{aligned}\right.
$$

is an isomorphism of categories. By Theorem 5.12, the functor

$$
E: \begin{cases}Z_{Q^{-}}(T-\mathcal{C}) & \longrightarrow Z_{Q}(T-\mathcal{C}) \\ ((M, r), s) & \longmapsto\left((M, r),\left(\operatorname{id}_{Q} \otimes s\right) \tilde{\partial}_{(M, r), 1_{T^{-}}}\right)\end{cases}
$$

is an isomorphism of categories. Using Theorem $7.4(\mathrm{a})$, one verifies that $I=E L$. Thus $I$ is an isomorphism of categories, and it clearly satisfies $\mathcal{U} I=U_{D_{Q, T}}$.

Assume $Q$ is a bimonad. Then $L$ is strict monoidal (by Theorem 4.7) and $E$ is strict monoidal (by Theorem 5.12). Hence $I=E L$ is strict monoidal, and so $\mathcal{U} I=U_{D_{Q, T}}$ as monoidal functors (since $\mathcal{U}$ and $U_{D_{Q, T}}$ are strict monoidal).

The canonical distributive law $\Omega$ can be described explicitly as follows. By Proposition 5.3, we have

$$
Z_{Q \rtimes T}(X)=\int^{Y \in \mathcal{C}} \vee Q \rtimes T(Y) \otimes X \otimes Y
$$

with universal dinatural transformation

$$
i_{X, Y}=\left(\operatorname{ev}_{Q \rtimes T(Y)} \otimes \operatorname{id}_{Z_{Q \rtimes T}(X)}\right)\left(\operatorname{id}_{Q \rtimes T(Y)} \otimes \partial_{X, Y}\right) .
$$

Recall that $T(i)$ is a universal dinatural transformation (see Proposition 3.10). Denote by $s^{l}$ the left antipode of $T$ and by $\varepsilon$ the counit of the adjunction $\left(F_{T}, U_{T}\right)$.

Proposition 7.8. The canonical distributive law $\Omega$ of the pair $(Q, T)$ is invertible, and $\Omega$ and $\Omega^{-1}$ are characterized as natural transformations by

$$
\begin{aligned}
& \Omega_{X} T\left(i_{X, Y}\right)=i_{T(X), T(Y)}\left({ }^{\vee} b_{Y} s_{Y}^{l} T\left({ }^{\vee} a_{Y}\right) \otimes \operatorname{id}_{T(X) \otimes T(Y)}\right) T_{3}\left({ }^{\vee} Q \rtimes T(Y), X, Y\right), \\
& \Omega_{X}^{-1} i_{T(X), Y}=\left(\operatorname{ev}_{Q \rtimes T(Y)} \otimes T\left(i_{X, T(Y)}\right) \otimes \operatorname{ev}_{Y}\right)\left(\operatorname{id} \vee_{Q \rtimes T(Y)} \otimes E_{X, Y} \otimes \operatorname{id}_{Y}\right),
\end{aligned}
$$


where $a_{Y}=U_{T}\left(\varepsilon_{Q F_{T}(Y)}\right), b_{Y}=U_{T} Q\left(\varepsilon_{F_{T}(Y)}\right)$, and

$$
\begin{aligned}
E_{X, Y}= & \left(a_{Y} T\left(b_{Y}\right) \otimes \operatorname{id}_{T\left({ }^{\vee} Q \rtimes T T(Y) \otimes X \otimes T(Y)\right)} \otimes s_{Y}^{l}\right) \\
& \circ T_{3}\left(Q \rtimes T T(Y),{ }^{\vee} Q \rtimes T T(Y) \otimes X \otimes T(Y),{ }^{\vee} T(Y)\right) \\
& \circ T\left(\operatorname{coev}_{Q \rtimes T T(Y)} \otimes \operatorname{id}_{X} \otimes \operatorname{coev}_{T(Y)}\right) .
\end{aligned}
$$

Remark 7.9. In the special case $Q=1_{T-\mathcal{C}}$, we have $1_{T-\mathcal{C}} \rtimes T=T$ and so, by Proposition 7.8 the canonical distributive law of the pair $\left(1_{T-\mathcal{C}}, T\right)$ is nothing but the canonical law of $T$ defined in Section 6.1 and the double $D_{1_{T-\mathcal{C}}, T}$ of the pair $\left(1_{T-\mathcal{C}}, T\right)$ coincides with the double $D_{T}$ of $T$ defined in Section 6.2

Proof. Note that $a_{Y}$ and $a_{Y}^{\prime}=s_{Q \rtimes T(Y)}^{l} T\left({ }^{\vee} a_{Y}\right)$ are the $T$-actions of $Q F_{T}(Y)$ and ${ }^{\vee} Q F_{T}(Y)$, respectively. By adjunction we have $b_{Y} Q \rtimes T\left(\eta_{Y}\right)=\operatorname{id}_{Q \rtimes T(Y)}$.

Recall that $\tilde{Z}_{Q \rtimes T}^{\Omega}$ is the centralizer of $Q$, with universal dinatural transformation

$$
j_{(M, r),(N, s)}=i_{M, N}\left({ }^{\vee} U_{T} Q\left(\varepsilon_{(N, s)}\right) \otimes \operatorname{id}_{M} \otimes \operatorname{id}_{N}\right) .
$$

In particular, given two objects $X, Y$ of $\mathcal{C}$, the morphism $j_{F_{T}(X), F_{T}(Y)}$ is $T$-linear, that is,

where

$$
Z_{Q \rtimes T}\left(\mu_{X}\right) \Omega_{T(X)} T\left(j_{F_{T}(X), F_{T}(Y)}\right)=j_{F_{T}(X), F_{T}(Y)} \gamma_{X, Y},
$$

$$
\gamma_{X, Y}=\left(a_{Y}^{\prime} \otimes \mu_{X} \otimes \mu_{Y}\right) T_{3}\left({ }^{\vee} Q \rtimes T(Y), T(X), T(Y)\right)
$$

is the $T$-action of ${ }^{\vee} Q F_{T}(Y) \otimes F_{T}(X) \otimes F_{T}(Y)$. Composing on the right with $T\left(\operatorname{id}_{Q \rtimes T(Y)} \otimes \eta_{X} \otimes \eta_{Y}\right)$, the left-hand side becomes

$$
\begin{aligned}
& Z_{Q \rtimes T}\left(\mu_{X}\right) \Omega_{T(X)} T\left(i_{T(X), T(Y)}\left({ }^{\vee} b_{Y} \otimes \eta_{X} \otimes \eta_{Y}\right)\right) \\
& \quad=Z_{Q \rtimes T}\left(\mu_{X}\right) \Omega_{T(X)} T Z_{Q \rtimes T}\left(\eta_{X}\right) T\left(i_{X, Y}\right)=\Omega_{X} T\left(i_{X, Y}\right),
\end{aligned}
$$

and the right-hand side becomes

$$
i_{T(X), T(Y)}\left({ }^{\vee} b_{Y} a_{Y}^{\prime} \otimes \mu_{X} T\left(\eta_{X}\right) \otimes \mu_{Y} T\left(\eta_{Y}\right)\right) T_{3}\left({ }^{\vee} Q \rtimes T(Y), X, Y\right) .
$$

Hence the formula for $\Omega$.

Let $\Omega^{\prime}: Z_{Q \rtimes T} T \rightarrow T Z_{Q \rtimes T}$ be the natural transformation defined by

$$
\Omega_{X}^{\prime} i_{T(X), Y}=\left(\operatorname{ev}_{Q \rtimes T(Y)} \otimes T\left(i_{X, T(Y)}\right) \otimes \mathrm{ev}_{Y}\right)\left(\mathrm{id}_{Q \rtimes T(Y)} \otimes E_{X, Y} \otimes \operatorname{id}_{Y}\right) .
$$

Using the axioms of a left antipode, one shows that $\Omega^{\prime} \Omega=\operatorname{id}_{T Z_{Q \rtimes T}}$ and $\Omega^{\prime} \Omega=$ $\operatorname{id}_{Z_{Q \rtimes T} T}$ by verifying that $\Omega_{X}^{\prime} \Omega_{X} T\left(i_{X, Y}\right)=T\left(i_{X, Y}\right)$ and $\Omega_{X} \Omega_{X}^{\prime} i_{T(X), Y}=i_{T(X), Y}$. This is left to the reader. Note that when $Q$ is a Hopf monad, the invertibility of $\Omega$ follows from Proposition 4.12, since in this case both $T$ and $Z_{Q \rtimes T}$ are Hopf monads.

Remark 7.10. Let $T$ be a Hopf monad on an autonomous category $\mathcal{C}$ and $Q$ be a comonoidal endofunctor of $T$ - $\mathcal{C}$ such that $Q \rtimes T$ is centralizable. Consider the following diagram:

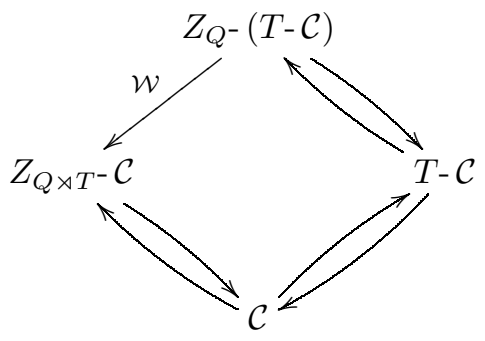


where a double arrow represents the adjunction of the corresponding monad and the functor $\mathcal{W}$ is defined by $\mathcal{W}((M, r), s)=(M, s)$ and $\mathcal{W}(f)=f$. This diagram is a distributive adjoint square in the sense of Beck [Bec69] whose distributive law is precisely the canonical distributive law $\Omega$ of the pair $(Q, T)$. Furthermore, since $\Omega$ is invertible by Proposition 7.8 , the monad $T$ lifts to a monad $\tilde{T}^{\Omega^{-1}}$ on $Z_{Q \rtimes T^{-}} \mathcal{C}$. Therefore, since $Z_{Q}=\tilde{Z}_{Q \rtimes T}^{\Omega}$, we have an isomorphism of categories:

$$
\tilde{T}^{\Omega^{-1}}-\left(Z_{Q \rtimes T^{-}} \mathcal{C}\right) \simeq\left(T \circ_{\Omega^{-1}} Z_{Q \rtimes T}\right)-\mathcal{C} \simeq\left(Z_{Q \rtimes T} \circ_{\Omega} T\right)-\mathcal{C} \simeq Z_{Q^{-}}(T-\mathcal{C}) .
$$

Via this isomorphism, $\mathcal{W}$ is the forgetful functor $U_{\tilde{T}^{\Omega^{-1}}}$. Hence $\mathcal{W}$ is monadic. Note that when $Q$ is a bimonad, the four monadic adjunctions are comonoidal.

7.4. Proof of Theorem 6.1. This is a direct consequence of Remark 7.9 and Propositions 7.5 and 7.8 applied to the Hopf monad $Q=1_{T-\mathcal{C}}$.

7.5. Proof of Theorems 6.4 and 6.5. By Theorem 7.6 applied to the Hopf monad $Q=1_{T-\mathcal{C}}$ and Remark 7.9 , the functor $I: D_{T^{-}} \mathcal{C} \rightarrow \mathcal{Z}(T-\mathcal{C})$ of Theorem 6.5 is a strict monoidal isomorphism of monoidal categories such that $\mathcal{U} I=U_{D_{T}}$.

Now, by Remark 5.10, the category $\mathcal{Z}(T-\mathcal{C})$ is a braided category with braiding:

$$
\tau_{((M, r), \gamma),((N, s), \delta)}=\gamma_{(N, s)}
$$

Therefore, since $I$ is a strict monoidal isomorphism, there exists a unique braiding $c$ on $D_{T^{-}} \mathcal{C}$ such that $I$ is braided. By Theorem 2.11, $c$ is encoded by an R-matrix $R$ for $D_{T}$. Let $p$ and $e=u_{T} \eta$ be the product and unit of $D_{T}$. Then $R$ is given by

$$
\begin{aligned}
R_{X, Y} & =c_{F_{D_{T}(X)}, F_{D_{T}(Y)}}\left(e_{X} \otimes e_{Y}\right) \\
& =\tau_{I F_{D_{T}(X)}, I F_{D_{T}(Y)}}\left(e_{X} \otimes e_{Y}\right) \\
& =\left(p_{Y} u_{D_{T}(Y)} \otimes p_{X} Z_{T}\left(\eta_{D_{T}(X)}\right)\right) \partial_{D_{T}(X), D_{T}(Y)}\left(e_{X} \otimes e_{Y}\right) \\
& =\left(p_{Y} u_{D_{T}(Y)} T\left(e_{Y}\right) \otimes p_{X} Z_{T}\left(\eta_{D_{T}(X)} e_{X}\right)\right) \partial_{X, Y} \\
& =\left(p_{Y} D_{T}\left(e_{Y}\right) u_{T(Y)} \otimes p_{X} D_{T}\left(e_{X}\right) Z_{T}\left(\eta_{X}\right)\right) \partial_{X, Y} \\
& =\left(u_{T(Y)} \otimes Z_{T}\left(\eta_{X}\right)\right) \partial_{X, Y} .
\end{aligned}
$$

This concludes the proof of Theorems 6.4 and 6.5 .

7.6. Proof of Theorem 6.9. This is a direct consequence of Remark 7.9 and Theorem 7.4 applied to the Hopf monad $Q=1_{T-\mathcal{C}}$.

\section{The double of A Hopf Algebra in A BRAided CATEgory}

In this section, we extend several classical notions concerning a Hopf algebra over a field to a Hopf algebra $A$ in a braided autonomous category $\mathcal{B}$, namely, quasitriangularity and R-matrices and the double $D(A)$ of $A$. Our approach consists in applying the results of previous sections to the Hopf monad ? $\otimes A$.

We need to assume that $\mathcal{B}$ admits a coend $C$. Then the Hopf monad ? $\otimes A$ is centralizable, and its centralizer is of the form ? $\otimes Z(A)$, where $Z(A)$ is a certain Hopf algebra in $\mathcal{B}$ called the centralizer of $A$. As an object of $\mathcal{B}, Z(A)={ }^{\vee} A \otimes C$.

We then define the double of $A$ as $D(A)=A \otimes_{\Omega} Z(A)=A \otimes{ }^{\vee} A \otimes C$, where $\Omega$ is an explicit distributive law. The double $D(A)$ is a quasitriangular Hopf algebra in $\mathcal{B}$ such that $D_{? \otimes A}=? \otimes D(A)$ (as quasitriangular Hopf monads). It satisfies $\mathcal{Z}\left(\mathcal{B}_{A}\right) \simeq \mathcal{B}_{D(A)}$ (as braided categories). When $\mathcal{B}=$ vect $_{\mathbb{k}}$, we have that $C=\mathbb{k}, A$ 
is a finite-dimensional Hopf algebra over $\mathbb{k}, Z(A)=\left(A^{*}\right)^{\text {cop }}$, and $D(A)$ is the usual Drinfeld double of $A$.

8.1. Hopf monads represented by Hopf algebras. Let $\mathcal{B}$ be a braided autonomous category and let $A$ be a Hopf algebra in $\mathcal{B}$. A Hopf monad $T$ on $\mathcal{B}$ is said to be represented on the left (resp. on the right) by $A$ if it is isomorphic to the Hopf monad $A \otimes$ ? (resp. ? $\otimes A$ ) defined in Example 2.4

More generally, let $T$ be a Hopf monad on an autonomous category $\mathcal{C}$. If $(A, \sigma)$ is a Hopf algebra in the center $\mathcal{Z}(\mathcal{C})$ of $\mathcal{C}$, then the Hopf monad $T$ is said to be represented on the left by $(A, \sigma)$ if it is isomorphic to the Hopf monad $A \otimes_{\sigma}$ ? on $\mathcal{C}$ defined in Example 2.5. Likewise, if $(A, \sigma)$ is a Hopf algebra in $\mathcal{Z}^{\prime}(\mathcal{C})$, then the Hopf monad $T$ is said to be represented on the right by $(A, \sigma)$ if it is isomorphic to the Hopf monad $? \otimes_{\sigma} A$ on $\mathcal{C}$.

Not all Hopf monads can be so represented by Hopf algebras (see Remark 8.5 for an example).

8.2. Coends as Hopf algebras. Let $T$ be an endofunctor of an autonomous category $\mathcal{C}$. If $\mathcal{C}$ admits a braiding $\tau$, then, by Proposition 5.3, $T$ is centralizable if and only if the coend

$$
C_{T}=\int^{Y \in \mathcal{B}}{ }^{\vee} T(Y) \otimes Y
$$

exists. Assume this is the case. By Lemma 3.9, if $T$ is a monad, then $C_{T}$ coincides with the coend $\int^{(M, r) \in T-\mathcal{C}} \vee_{U_{T}}(M, r) \otimes U_{T}(M, r)$ of $U_{T}$. According to Majid Maj95, the (co)end of a strong monoidal functor from an autonomous category to a braided category is a Hopf algebra. In particular, if $T$ is a Hopf monad and $\tau$ a braiding on $\mathcal{C}$, then $C_{T}$ is a Hopf algebra in $\mathcal{C}$ braided by $\tau$. In this section we recover this structure explicitly in terms of the braiding $\tau$ and the Hopf monad structure of $T$.

Let $T$ be an endofunctor of an autonomous category $\mathcal{C}$ such that the coend

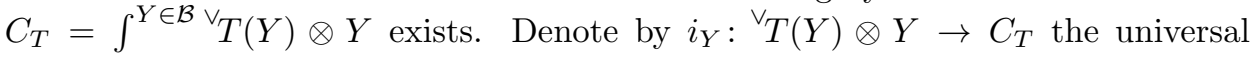
dinatural transformation of $C_{T}$, and set

$$
\delta_{Y}=\underbrace{T(Y)}_{Y} \sum_{i_{Y}}^{C_{T}}=\left(\operatorname{id}_{T(Y)} \otimes i_{Y}\right)\left(\operatorname{coev}_{T(Y)} \otimes \operatorname{id}_{Y}\right): X \rightarrow T(Y) \otimes C_{T} \text {, depicted as } \bigsqcup_{Y}^{T(Y)} C_{T}^{C_{T}} .
$$

If $T$ is a monad on $\mathcal{C}$, then $C_{T}$ is a coalgebra in $\mathcal{C}$, with coproduct $\Delta$ and counit $\varepsilon$ defined by

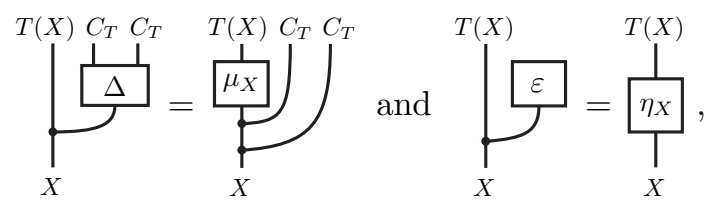

where $\mu$ and $\eta$ are the product and unit of $T$. 
If $T$ is comonoidal and $\tau$ is a braiding on $\mathcal{C}$, then $C_{T}$ becomes an algebra in $\mathcal{C}$ with product $m_{\tau}$ and unit $u$ defined by

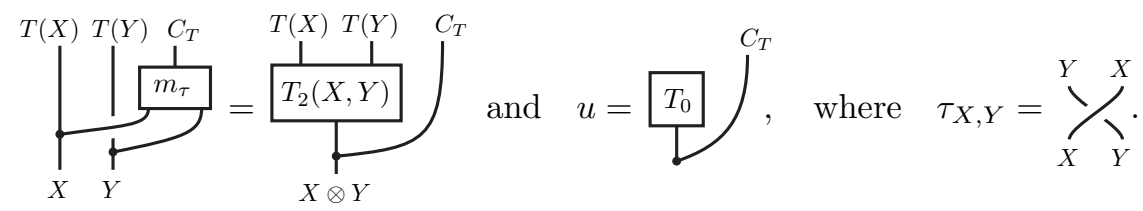

If $T$ is a bimonad and $\tau$ a braiding on $\mathcal{C}$, then $\left(C_{T}, m^{\tau}, u, \Delta, \varepsilon\right)$ is a bialgebra in $\mathcal{C}$ braided by $\tau$. Furthermore, if $T$ is a Hopf monad, then $C_{T}$ is a Hopf algebra, whose antipode $S_{\tau}$ and its inverse $S_{\tau}^{-1}$ are defined by

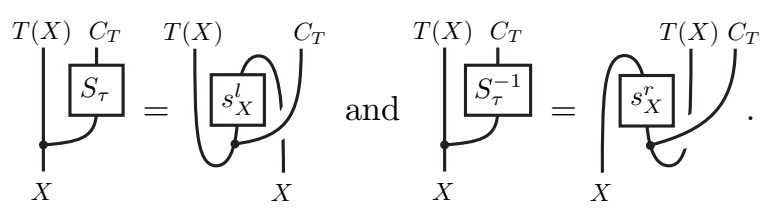

We denote this Hopf algebra by $C_{T}^{\tau}$.

8.3. The coend of a braided autonomous category. Let $\mathcal{B}$ be an autonomous category. The coend

$$
C=\int^{Y \in \mathcal{B}}{ }^{\vee} Y \otimes Y
$$

if it exists, is called the coend of $\mathcal{B}$.

Assume that $\mathcal{B}$ admits a coend $C$ and denote by $i_{Y}:{ }^{\vee} Y \otimes Y \rightarrow C$ its universal dinatural transformation. The universal coaction of $C$ on the objects of $\mathcal{B}$ is the natural transformation $\delta$ defined by

$$
\delta_{Y}=\left(\operatorname{id}_{Y} \otimes i_{Y}\right)\left(\operatorname{coev}_{Y} \otimes \operatorname{id}_{Y}\right): Y \rightarrow Y \otimes C, \quad \text { depicted as } \delta_{Y}=\zeta_{Y}^{Y} .
$$

If $\mathcal{B}$ is braided, then $C$ is a Hopf algebra in $\mathcal{B}$. This well-known fact may be viewed as a special case of the construction of Section 8.4 as $1_{\mathcal{B}}$ is a Hopf monad on $\mathcal{B}$ and $C=C_{1_{\mathcal{B}}}^{\tau}$, where $\tau$ is the braiding of $\mathcal{B}$. Furthermore, the morphism $\omega: C \otimes C \rightarrow \mathbb{1}$, defined by

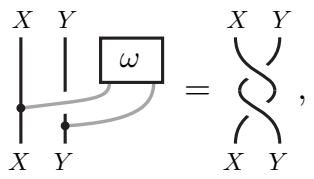

is a Hopf pairing for $C$, that is, it satisfies

$$
\begin{aligned}
& \omega\left(m \otimes \operatorname{id}_{C}\right)=\omega\left(\operatorname{id}_{C} \otimes \omega \otimes \operatorname{id}_{C}\right)\left(\operatorname{id}_{C \otimes 2} \otimes \Delta\right), \quad \omega\left(u \otimes \operatorname{id}_{C}\right)=\varepsilon, \\
& \omega\left(\mathrm{id}_{C} \otimes m\right)=\omega\left(\mathrm{id}_{C} \otimes \omega \otimes \mathrm{id}_{C}\right)\left(\Delta \otimes \operatorname{id}_{C \otimes 2}\right), \quad \omega\left(\mathrm{id}_{C} \otimes u\right)=\varepsilon .
\end{aligned}
$$

These axioms imply $\omega\left(S \otimes \operatorname{id}_{C}\right)=\omega\left(\operatorname{id}_{C} \otimes S\right)$. Moreover, the pairing $\omega$ satisfies the self-duality condition $\omega \tau_{C, C}(S \otimes S)=\omega$.

In this section, the structural morphisms of $C$ are drawn in grey and the Hopf pairing $w: C \otimes C \rightarrow \mathbb{1}$ is depicted as

$$
\omega=\bigcap_{C} \text {. }
$$


Remark 8.1. The category $\mathcal{B}$ is symmetric if and only if $\omega=\epsilon \otimes \epsilon$. In particular, this is the case when $C=\mathbb{1}$.

Remark 8.2. The universal coaction of the coend on itself can be expressed in terms of its Hopf algebra structure as follows:

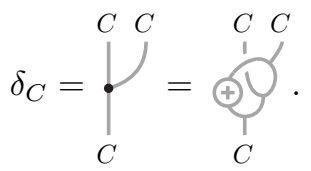

Remark 8.3. The coend of the mirror $\overline{\mathcal{B}}$ of $\mathcal{B}$ is the Hopf algebra $C^{\text {op }}$, with self-dual pairing $\omega\left(S \otimes \operatorname{id}_{C}\right)$.

8.4. Centralizers in braided categories. Let $T$ be an endofunctor of a braided autonomous category $\mathcal{B}$, with braiding $\tau$. Assume that the coend

$$
C_{T}=\int^{Y \in \mathcal{B}}{ }^{\vee} T(Y) \otimes Y
$$

exists. Set

$$
\partial_{X, Y}=\nearrow_{X}^{T(Y)} \int_{Y}^{X}=\left(\tau_{X, T(Y)}^{C_{T}} \otimes \operatorname{id}_{C_{T}}\right)\left(\operatorname{id}_{X} \otimes \delta_{Y}\right): X \otimes Y \rightarrow T(Y) \otimes X \otimes C_{T} .
$$

Then $\left(? \otimes C_{T}, \partial\right)$ is a centralizer of $T$. Likewise, set

$$
\partial_{X, Y}^{\prime}=\overbrace{X}^{T(Y) C_{Y}^{C_{T} X}}=\left(\delta_{Y} \otimes \operatorname{id}_{X}\right) \tau_{Y, X}^{-1}: X \otimes Y \rightarrow T(Y) \otimes C_{T} \otimes X .
$$

Then $\left(C_{T} \otimes\right.$ ?, $\left.\partial^{\prime}\right)$ is also a centralizer of $T$.

Assume furthermore that $T$ is a Hopf monad. By Section 8.2 the object $C_{T}$ is endowed with two Hopf algebra structures in $\mathcal{B}$, namely, $C_{T}^{\tau}$ and $\left(C_{T}^{\bar{\tau}}\right)^{\text {op }}$, where $\bar{\tau}$ is the mirror of $\tau$. One verifies that the Hopf monad structure on ? $\otimes C_{T}$ (resp. $C_{T} \otimes$ ?) given by Theorem 5.6 coincides with that induced by the Hopf algebra $C_{T}^{\tau}$ (resp. $\left.\left(C_{T}^{\bar{\tau}}\right)^{\text {op }}\right)$. Thus:

Theorem 8.4. Let $T$ be a Hopf monad on a braided autonomous category $\mathcal{B}$, with braiding $\tau$. Then $T$ is centralizable if and only if the coend

$$
C_{T}=\int^{Y \in \mathcal{B}} \vee T(Y) \otimes Y
$$

exists. If such is the case, the centralizer of $T$ is represented on the right by the Hopf algebra $C_{T}^{\tau}$ and on the left by the Hopf algebra $\left(C_{T}^{\bar{\tau}}\right)^{\mathrm{op}}$.

Remark 8.5. In general, the centralizer $Z_{T}$ of a Hopf monad $T$ on an autonomous category $\mathcal{C}$ is isomorphic neither to $Z_{T}(\mathbb{1}) \otimes$ ? nor to ? $\otimes Z_{T}(\mathbb{1})$ as an endofunctor of $\mathcal{C}$, see Remark 9.2 for a counterexample. In particular, it cannot be represented on the left by a Hopf algebra of $\mathcal{Z}(\mathcal{C})$ or on the right by a Hopf algebra of $\mathcal{Z}^{\prime}(\mathcal{C})$, in the sense of Section 8.1 
8.5. Centralizers of Hopf algebras. Let $\mathcal{B}$ be a braided autonomous category, with braiding $\tau$, admitting a coend $C=\int^{Y \in \mathcal{B} \vee} Y \otimes Y$. Recall that $C$ is a Hopf algebra in $\mathcal{B}$ endowed with a Hopf pairing, and denote by $\delta$ the universal coaction of $C$ (see Section 8.3).

Let $A$ be a Hopf algebra in $\mathcal{B}$. Set $Z(A)={ }^{\vee} A \otimes C$. Then

$$
Z(A)=\int^{Y \in \mathcal{B}} \vee(Y \otimes A) \otimes Y
$$

with universal dinatural transformation given by

$$
i_{Y}=\left.\right|_{\vee_{A}} ^{\vee_{A}} \bigcap_{\vee_{Y}}^{C}:{ }^{\vee}(Y \otimes A) \otimes Y={ }^{\vee} A \otimes{ }^{\vee} Y \otimes Y \rightarrow{ }^{\vee} A \otimes C .
$$

The object $Z(A)$ is endowed with the Hopf algebra structure $C_{? \otimes A}^{\tau}$ defined in Section 8.2 and is called the centralizer of $A$. Explicitly, the structural morphisms of $Z(A)$ are

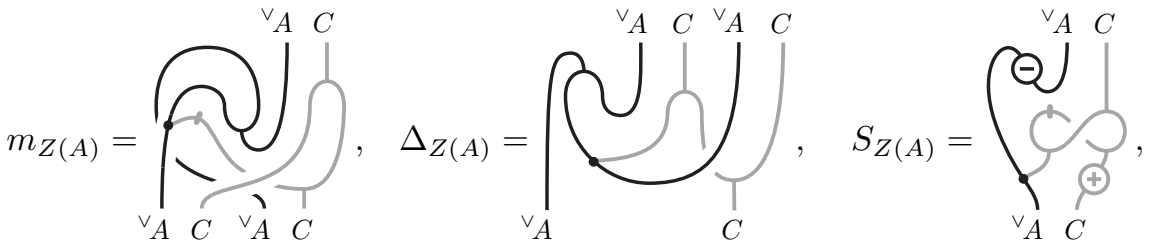

$$
\begin{aligned}
& u_{Z(A)}=q \int_{0}^{\vee_{A}}, \quad \varepsilon_{Z(A)}^{C}=\underbrace{0}_{\vee_{A}} \underbrace{}_{C} .
\end{aligned}
$$

Example 8.6. Let $H$ be a finite-dimensional Hopf algebra over a field $\mathbb{k}$. Note that $\mathbb{k}$ is the coend of the category vect $\mathbb{k}_{\mathbb{k}}$ of finite-dimensional vector spaces. Then the centralizer of $H$ is $Z(H)=\left(H^{*}\right)^{\mathrm{cop}}$.

By Theorem 8.4, $(? \otimes Z(A), \partial)$ is a centralizer of $? \otimes A$, where

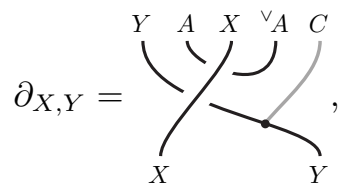

and the Hopf monad structure on ? $\otimes Z(A)$ given by Theorem [5.6 is that induced by the Hopf algebra $Z(A)$. Hence, by Theorem 5.12 , we have

$$
\mathcal{Z}_{? \otimes A}(\mathcal{B}) \simeq(? \otimes Z(A))-\mathcal{B}=\mathcal{B}_{Z(A)}
$$

as monoidal categories.

Remark 8.7. One can show that the Hopf algebra $Z(A)$ represents on the left the centralizer $Z_{A \otimes \text { ? }}$ of $A \otimes$ ?, and so

$$
\mathcal{Z}_{A \otimes ?}(\mathcal{B}) \simeq(Z(A) \otimes ?)-\mathcal{B}={ }_{Z(A)} \mathcal{B}
$$

as monoidal categories. 
8.6. R-matrices for Hopf algebras in braided categories. In [Dri90, Drinfeld introduced the notion of R-matrix for a Hopf algebra $H$ over a field $\mathbb{k}$. When $H$ is finite-dimensional, R-matrices for $H$ are in bijection with braidings on the category of finite-dimensional $H$-modules. The aim of this section is to extend the notion of an R-matrix to a Hopf algebra $A$ in braided autonomous category so as to preserve this bijective correspondence. Note that the definition of an R-matrix for $A$ as a morphism $\mathfrak{r}: \mathbb{1} \rightarrow A \otimes A$ by straightforward extension of Drinfeld's axioms (sometimes found in the literature) does not fulfil this objective. Recall that braidings on the autonomous category $\mathcal{B}_{A}=(? \otimes A)-\mathcal{B}$ are encoded by R-matrices for the Hopf monad ? $\otimes A$. When $\mathcal{B}$ admits a coend, we can encode R-matrices for $? \otimes A$ in terms of $A$, which leads to our definition of an R-matrix for $A$.

Let $A$ be a Hopf algebra in a braided autonomous category $\mathcal{B}$, with braiding $\tau$. Assume that $\mathcal{B}$ admits a coend $C$. Any R-matrix $R_{X, Y}: X \otimes Y \rightarrow Y \otimes A \otimes X \otimes A$ for the Hopf monad ? $\otimes A$ gives rise to a unique morphism $\mathfrak{r}: C \otimes C \rightarrow A \otimes A$ in $\mathcal{B}$, defined by

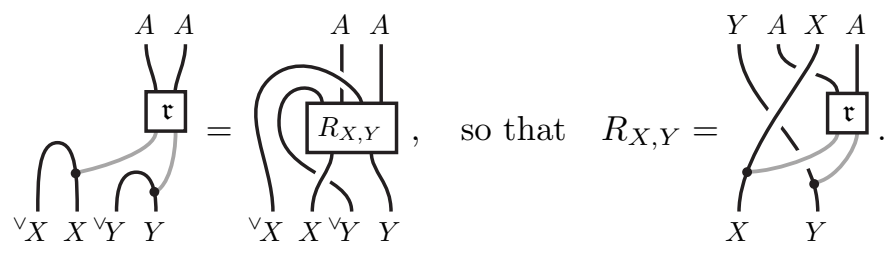

Re-writing the axioms for $R_{X, Y}$ (see Section 2.7) in terms of $\mathfrak{r}$ leads to the following definition: an R-matrix for $A$ is a morphism

$$
\mathfrak{r}: C \otimes C \rightarrow A \otimes A
$$

in $\mathcal{B}$ which satisfies
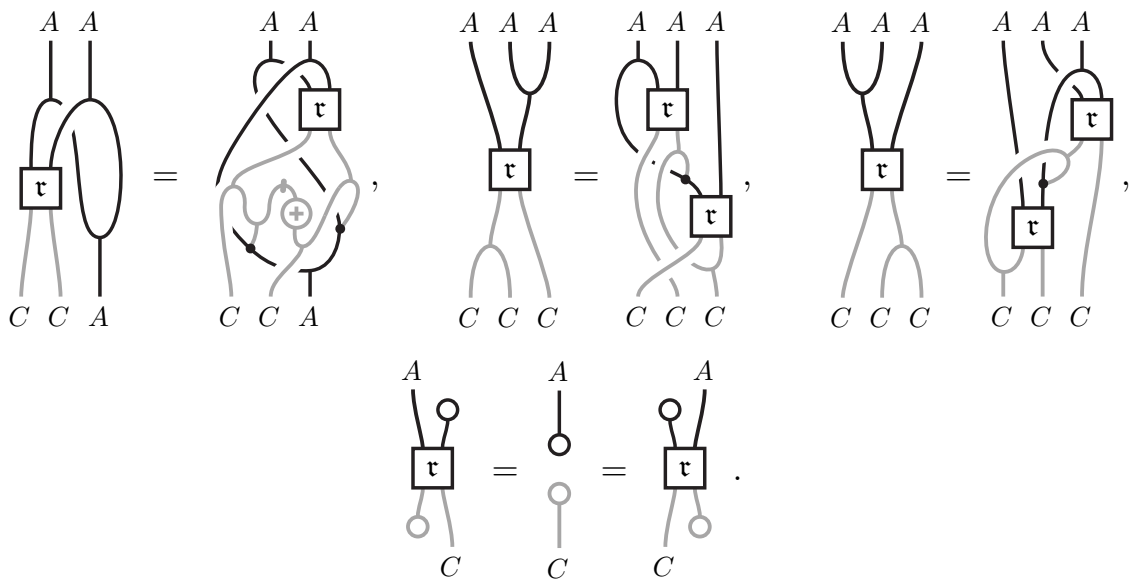

Remark 8.8. For finite-dimensional Hopf algebras over a field $\mathbb{k}$, our definition of an R-matrix coincides with Drinfeld's definition (as the coend of vect $\mathbb{k}_{\mathbb{k}}$ is $\mathbb{k}$ ). 
An R-matrix $\mathfrak{r}$ for $A$ defines an R-matrix for ? $\otimes A$ (by definition) and so a braiding $c$ on $\mathcal{B}_{A}=(? \otimes A)-\mathcal{B}$ (by Theorem 2.11):

$$
c_{(M, r),(N, s)}=(s \otimes r) R_{M, N}=\underbrace{r}_{M N}
$$

As braidings on ${ }_{A} \mathcal{B}$ are in bijective correspondence with braidings on $\mathcal{B}_{A}$ (see Remark 1.3), an R-matrix $\mathfrak{r}$ for $A$ also defines a braiding $c^{\prime}$ on ${ }_{A} \mathcal{B}$ as

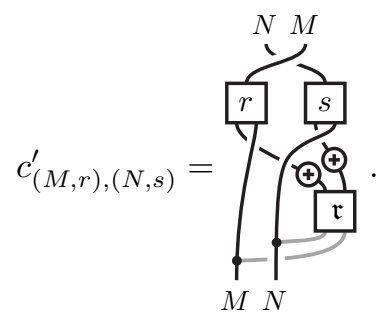

Furthermore, the map $\mathfrak{r} \mapsto c$ (resp. $\mathfrak{r} \mapsto c^{\prime}$ ) is a bijection between R-matrices for $A$ and braidings on $\mathcal{B}_{A}$ (resp. on ${ }_{A} \mathcal{B}$ ).

A quasitriangular Hopf algebra in $\mathcal{B}$ is a Hopf algebra in $\mathcal{B}$ endowed with an R-matrix.

Remark 8.9. Let $A$ be a quasitriangular Hopf algebra in $\mathcal{B}$. By construction, the monoidal isomorphism $F_{A}:\left({ }_{A} \mathcal{B}\right)^{\otimes \mathrm{op}} \rightarrow \mathcal{B}_{A}$ of Remark 1.3 is braided.

Remark 8.10. Let $A$ be a quasitriangular Hopf algebra in $\mathcal{B}$. Combining Remark 8.9 with Example 1.1 we obtain that ${ }_{A} \mathcal{B}$ and $\mathcal{B}_{A}$ are braided isomorphic.

8.7. The canonical distributive law of a Hopf algebra. Let $A$ be a Hopf algebra in a braided autonomous category $\mathcal{B}$ which admits a coend $C$. By Section 8.5 the centralizer of ? $\otimes A$ is $Z ? \otimes A=? \otimes Z(A)$, where $Z(A)={ }^{\vee} A \otimes C$ is the centralizer of $A$. It turns out that the canonical distributive law of ? $\otimes A$ over $Z$ ? $\otimes A$ is of the form $\operatorname{id}_{1_{\mathcal{B}}} \otimes \Omega$, where $\Omega: Z(A) \otimes A \rightarrow A \otimes Z(A)$ is a comultiplicative distributive law of $Z(A)$ over $A$ (see Example 4.3). We have

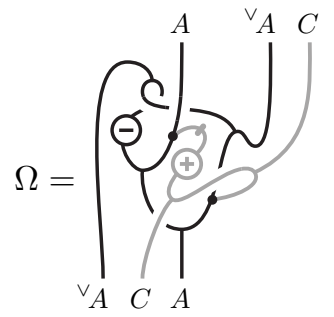

and

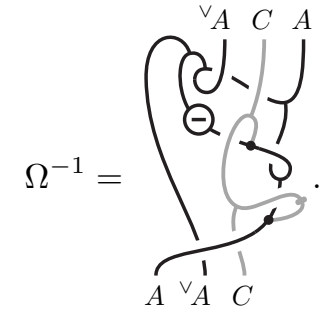

We call $\Omega$ the canonical distributive law of $A$.

Remark 8.11. By Theorem 6.9, $Z_{\mathcal{B}_{A}}(M, r)=\left(M \otimes Z(A),\left(r \otimes \operatorname{id}_{Z(A)}\right)\left(\operatorname{id}_{M} \otimes \Omega\right)\right)$ is the centralizer of the category $\mathcal{B}_{A}$. In particular, the coend of $\mathcal{B}_{A}$ is $Z_{\mathcal{B}_{A}}\left(\mathbb{1}, \varepsilon_{A}\right)=$ 
$(Z(A), \alpha)$, where

$$
\alpha=\left(\varepsilon_{A} \otimes \operatorname{id}_{Z(A)}\right) \Omega=\text { ( }_{\vee_{A}}
$$

If $A$ is quasitriangular, so that $\mathcal{B}_{A}$ is braided, then $(Z(A), \alpha)$ is a Hopf algebra in $\mathcal{B}_{A}$ which represents $Z_{\mathcal{B}_{A}}$ on the right (see Theorem 8.4). However, in this case, this Hopf algebra $(Z(A), \alpha)$ is not in general a lift to $\mathcal{B}_{A}$ of the Hopf algebra $Z(A)$.

8.8. The double of a Hopf algebra in a braided category. Let $A$ be a Hopf algebra in a braided autonomous category $\mathcal{B}$ which admits a coend $C$.

Let $Z(A)$ be the centralizer of $A$ (see Section 8.5) and $\Omega$ be the canonical distributive law of $A$ (see Section 8.7). By Example 4.3.

$$
D(A)=A \otimes_{\Omega} Z(A)=A \otimes{ }^{\vee} A \otimes C
$$

is a Hopf algebra in $\mathcal{B}$. Since ? $\otimes Z(A)$ is the centralizer of ? $\otimes A$ (see Section 8.5), the Hopf monad $? \otimes D(A)$ is the double of $? \otimes A$, and so admits an R-matrix by Theorem 6.5. which turns out to be encoded by the following R-matrix for $D(A)$ :

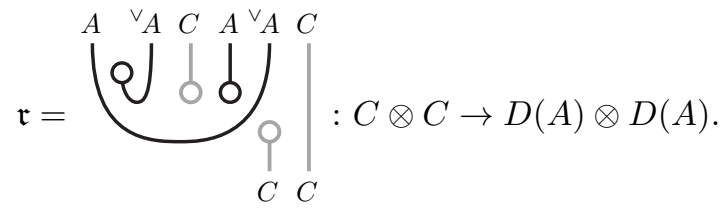

The quasitriangular Hopf algebra $D(A)$ is called the double of $A$.

Remark 8.12. The canonical distributive law of $A$ is the unique comultiplicative distributive law $\Omega$ of $Z(A)$ over $A$ such that the morphism $\mathfrak{r}$ above is an R-matrix for $A \otimes_{\Omega} Z(A)$, see Remark 6.8 .

Theorem 8.13. Let $A$ be a Hopf algebra in a braided autonomous category $\mathcal{B}$ admitting a coend $C$ and let $D(A)=A \otimes_{\Omega} Z(A)$ be the double of $A$. We have the following isomorphisms of braided categories:

$$
\mathcal{Z}\left(\mathcal{B}_{A}\right) \simeq \mathcal{B}_{D(A)} \simeq{ }_{D(A)} \mathcal{B} \simeq \mathcal{Z}^{\prime}\left({ }_{A} \mathcal{B}\right) \simeq \overline{\mathcal{Z}\left({ }_{A} \mathcal{B}\right)}
$$

Proof. By construction, the quasitriangular Hopf monad ? $\otimes D(A)$ is the double of ? $\otimes A$, hence the first braided isomorphism by Theorem 6.5. By Remark 8.10, $D(A) \mathcal{B} \simeq \mathcal{B}_{D(A)}$ as braided categories since $D(A)$ is quasitriangular. Finally, we have the following isomorphisms of braided categories:

$$
\begin{aligned}
\mathcal{Z}\left(\mathcal{B}_{A}\right) & \simeq \mathcal{Z}\left(\mathcal{B}_{A}\right)^{\otimes \mathrm{op}} \quad \text { by Remark } 1.1 \\
& \simeq \mathcal{Z}\left(\left({ }_{A} \mathcal{B}\right)^{\otimes \mathrm{op}}\right)^{\otimes \mathrm{op}} \quad \text { by Remark } 8.9 \\
& \simeq \mathcal{Z}^{\prime}\left({ }_{A} \mathcal{B}\right) \simeq \overline{\mathcal{Z}\left({ }_{A} \mathcal{B}\right)} \quad \text { by Remark } 1.2
\end{aligned}
$$

This completes the proof of the theorem.

Remark 8.14. When $\mathcal{B}=$ vect $_{\mathbb{k}}$ is the category of finite-dimensional vector spaces over a field $\mathbb{k}$, we recover the usual Drinfeld double and the interpretation of its category of modules in terms of the center. More precisely, let $H$ be a finitedimensional Hopf algebra over $\mathbb{k}$ and $\left(e_{i}\right)$ be a basis of $H$ with dual basis $\left(e^{i}\right)$. 
Then $D(H)=H \otimes\left(H^{*}\right)^{\text {cop }}$ is a quasitriangular Hopf algebra over $\mathbb{k}$, with R-matrix $\mathfrak{r}=\sum_{i} e_{i} \otimes \varepsilon \otimes 1_{H} \otimes e_{i}$, such that

$$
\mathcal{Z}\left(\left(\operatorname{vect}_{\mathbb{k}}\right)_{H}\right) \simeq\left(\text { vect }_{\mathbb{k}}\right)_{D(H)} \simeq{ }_{D(H)}\left(\operatorname{vect}_{\mathbb{k}}\right) \simeq \mathcal{Z}^{\prime}\left({ }_{H}\left(\text { vect }_{\mathbb{k}}\right)\right) \simeq \overline{\mathcal{Z}\left({ }_{H}\left(\text { vect }_{\mathbb{k}}\right)\right)}
$$

as braided categories.

Remark 8.15. By Remark 4.2, $\Omega^{-1}$ is a distributive law of $Z(A)$ over $A$ and induces an isomorphism of Hopf algebras

$$
D(A)=A \otimes_{\Omega} Z(A) \stackrel{\sim}{\longrightarrow} Z(A) \otimes_{\Omega^{-1}} A .
$$

Via this isomorphism, the R-matrix $\mathfrak{r}$ of $D(A)$ is sent to the R-matrix

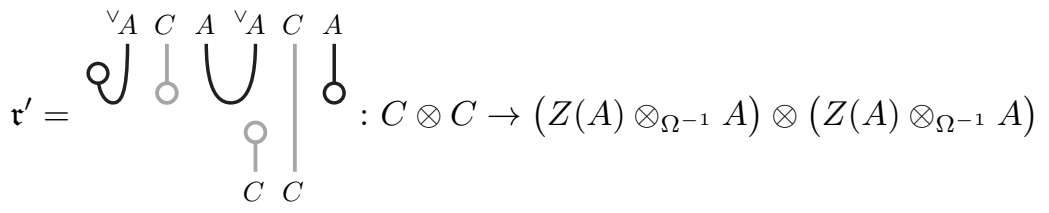

of $Z(A) \otimes_{\Omega^{-1}} A$.

Remark 8.16. Let $\mathcal{B}$ be a braided autonomous category which admits a coend $C$. Then $D(\mathbb{1})=C$ as a Hopf algebra. Hence $C$ is quasitriangular, with R-matrix $\mathfrak{r}=u_{C} \varepsilon_{C} \otimes \operatorname{id}_{C}$, and $\mathcal{Z}(\mathcal{B}) \simeq \mathcal{B}_{C} \simeq{ }_{C} \mathcal{B} \simeq \overline{\mathcal{Z}(\mathcal{B})}$ as braided categories. In other words, the center of $\mathcal{B}$ is self-mirror and is the category of $C$-modules in $\mathcal{B}$.

\section{HOPF MONADS AND FUSION CATEGORIES}

In this section, given a $\mathbb{k}$-linear Hopf monad $T$ of a fusion category $\mathcal{F}$, we describe explicitly the centralizer of $T$ and the canonical distributive law of $T$. Hence, in particular, a description of the coend of $\mathcal{Z}(\mathcal{F})$, which is used in BV10 to show that the center of a spherical fusion category is modular and in BV08 to provide an algorithm for computing the Reshetikhin-Turaev invariant $\mathrm{RT}_{\mathcal{Z}(\mathcal{F})}$ in terms of $\mathcal{F}$ itself.

9.1. Fusion categories. A fusion category $\mathcal{F}$ over a commutative ring $\mathbb{k}$ is a $\mathbb{k}$ linear 4 autonomous category whose monoidal product $\otimes$ is $\mathbb{k}$-bilinear and such that there exists a finite set $I$ of objects of $\mathcal{F}$ satisfying:

- $\operatorname{Hom}_{\mathcal{F}}(i, j)=\delta_{i, j} \mathbb{k}$ for all $i, j \in I$;

- $\mathbb{1} \in I$

- each object of $\mathcal{F}$ is a finite direct sum of elements of $I$.

The set $I$ is then a representative set of scalar objects of $\mathcal{F}$ (an object $X$ of $\mathbb{k}$-linear category is said to be scalar if $\operatorname{End}(X)=\mathbb{k}$ ). The Hom spaces in $\mathcal{F}$ are free $\mathbb{k}$-modules of finite rank. The multiplicity of $i \in I$ in an object $X$ of $\mathcal{F}$ is defined as

$$
N_{X}^{i}=\operatorname{rank}_{\mathbb{k}} \operatorname{Hom}_{\mathcal{F}}(i, X)=\operatorname{rank}_{\mathbb{k}_{\mathrm{k}}} \operatorname{Hom}_{\mathcal{F}}(X, i) .
$$

For each object $X$ of $\mathcal{F}$, we choose families of morphisms $\left(p_{X}^{i, \alpha}: X \rightarrow i\right)_{1 \leq \alpha \leq N_{X}^{i}}$ and $\left(q_{X}^{i, \alpha}: i \rightarrow X\right)_{1 \leq \alpha \leq N_{X}^{i}}$ such that

$$
\operatorname{id}_{X}=\sum_{\substack{i \in I \\ 1 \leq \alpha \leq N_{X}^{i}}} q_{X}^{i, \alpha} p_{X}^{i, \alpha} \quad \text { and } \quad p_{X}^{i, \alpha} q_{X}^{j, \beta}=\delta_{i, j} \delta_{\alpha, \beta} \operatorname{id}_{i} .
$$

\footnotetext{
${ }^{4}$ In particular, finite direct sums exist in $\mathcal{F}$.
} 
9.2. Centralizers in fusion categories. Let $\mathcal{F}$ be a fusion category over a commutative ring $\mathbb{k}$ and $T$ be a $\mathbb{k}$-linear endofunctor $T$ of $\mathcal{F}$. Then $T$ is centralizable, with centralizer $\left(Z_{T}, \partial\right)$ given by

$$
Z_{T}(X)=\bigoplus_{i \in I}^{\vee} T(i) \otimes X \otimes i
$$

and

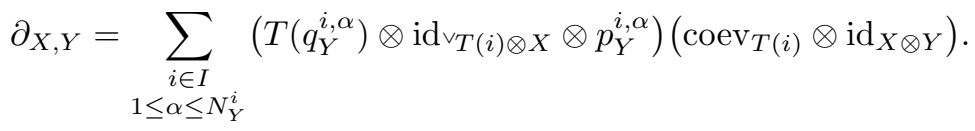

In particular, a fusion category is centralizable, with centralizer $Z=Z_{1_{\mathcal{F}}}$ given by

$$
Z(X)=\bigoplus_{i \in I} \vee i \otimes X \otimes i
$$

Remark 9.1. By Corollary 5.14, the centralizer $Z$ of $\mathcal{F}$ provides in particular a left adjoint $F_{Z}$ to the forgetful functor $\mathcal{U}: \mathcal{Z}(\mathcal{F}) \simeq Z-\mathcal{F} \rightarrow \mathcal{F}$, which is called the induction functor in ENO05.

Remark 9.2. In general, the centralizer $Z$ of $\mathcal{F}$ is not isomorphic (as an endofunctor of $\mathcal{F})$ to $Z(\mathbb{1}) \otimes$ ? or to ? $\otimes Z(\mathbb{1})$, as shown by the following counterexample. Let $G$ be a non-commutative finite group and let $\mathcal{F}$ be the fusion category of finitedimensional $G$-graded vector spaces over a field $\mathbb{k}$. The elements of $G$ form a representative set of scalar objects of $\mathcal{F}$. Then $Z(x)=\bigoplus_{g \in G} g^{-1} x g$ for $x \in G$. In particular, $Z(\mathbb{1})=\mathbb{1}^{\# G}$. Now, if $x \in G$ is not central, $Z(x)$ is not isomorphic to $Z(\mathbb{1}) \otimes x \simeq x^{\# G} \simeq x \otimes Z(\mathbb{1})$.

Assume $T$ is a centralizable Hopf monad. By Theorem 5.6, its centralizer $Z_{T}$ is a Hopf monad on $\mathcal{F}$ and its structural morphisms can be described purely in terms of those of $T$ and of the category $\mathcal{F}$ (that is, the $p, q$ 's and the duality morphisms). They are depicted in Figure 3, where $\mu, \eta, s^{l}, s^{r}$ (resp. $m, u, S^{l}, S^{r}$ ) denote the product, unit, left antipode, and right antipode of $T$ (resp. $\left.Z_{T}\right)$. The canonical distributive law of $T$ is

$$
\Omega_{X}=\sum_{\substack{i, j \in I \\ 1 \leq \alpha \leq N_{T(i)}^{j}}}\left({ }^{\vee} T\left(q_{T(i)}^{j, \alpha}\right){ }^{\vee} \mu_{i} s_{T(i)}^{l} T\left({ }^{\vee} \mu_{i}\right) \otimes \operatorname{id}_{T(X)} \otimes p_{T(i)}^{j, \alpha}\right) T_{3}\left({ }^{\vee} T(i), X, i\right) .
$$

Hence, we have an explicit description of the double $D_{T}=Z_{T} \circ_{\Omega} T$ of $T$ and of the lift $\tilde{Z}_{T}^{\Omega}$ of $Z_{T}$ to $T-\mathcal{F}$. Note that the R-matrix of $D_{T}$ is

$$
R_{X, Y}=\sum_{\substack{i \in I \\ 1 \leq \alpha \leq N_{Y}^{i}}}\left({ }^{\vee} T_{0} \otimes T\left(q_{Y}^{i, \alpha}\right) \otimes \operatorname{id} \vee_{T(i)} \otimes \eta_{X} \otimes p_{Y}^{i, \alpha}\right)\left(\operatorname{coev}_{T(i)} \otimes \operatorname{id}_{X \otimes Y}\right) .
$$

9.3. The coend of the center of a fusion category. Let $\mathcal{F}$ be a fusion category over a commutative ring $\mathbb{k}$, and denote by $Z$ the centralizer of $\mathcal{F}$. Recall that $Z$ is a quasitriangular Hopf monad on $\mathcal{F}$ such that $\mathcal{Z}(\mathcal{F}) \simeq Z-\mathcal{F}$, see Section 9.2 . Since $Z$ is $\mathbb{k}$-linear, it is centralizable. Denote by $Z_{Z}$ its centralizer and by $\Omega$ the canonical distributive law of $Z$ over $Z_{Z}$. Then the coend of $Z(\mathcal{F})$ is

$$
C=\tilde{Z}_{Z}^{\Omega}\left(\mathbb{1}, Z_{0}\right)=\left(Z_{Z}(\mathbb{1}), Z_{Z}\left(Z_{0}\right) \Omega_{\mathbb{1}}\right) .
$$




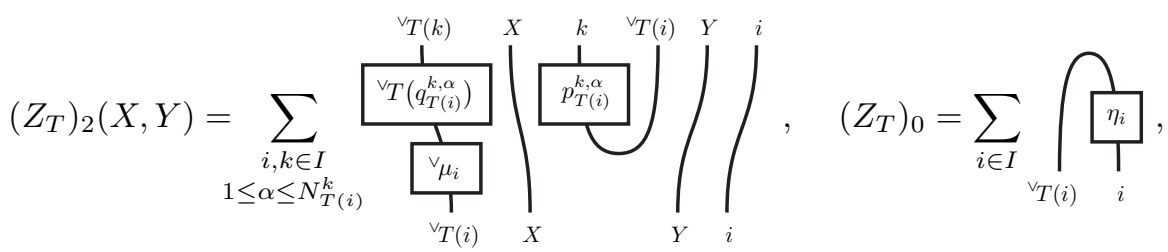

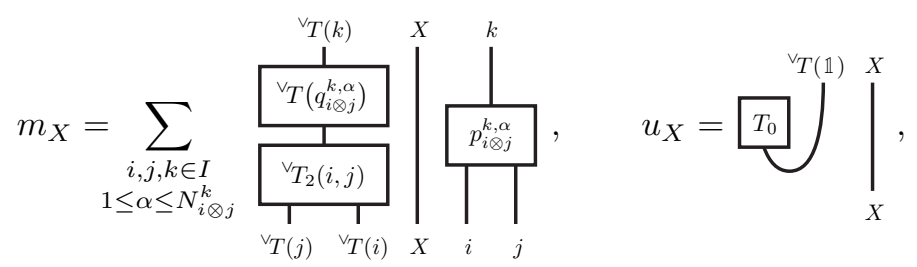

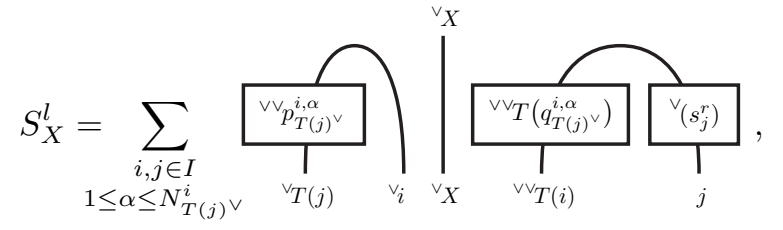

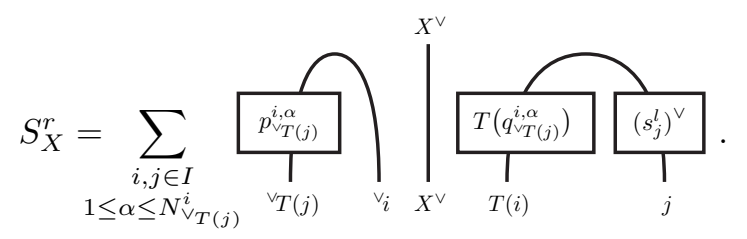

FIGURE 3. Structural morphisms of $Z_{T}$

Note that

$$
Z_{Z}(\mathbb{1})=\bigoplus_{j \in I}{ }^{\vee} Z(j) \otimes j=\bigoplus_{i, j \in I} \vee^{\vee} \otimes{ }^{\vee} j \otimes{ }^{\vee}{ } i \otimes j
$$

Using the results of Section 6.3. one computes the Hopf algebra structure of $C$ and its self-dual Hopf pairing. These are depicted in Figure 4, where the dotted lines represent the relevant canonical isomorphisms between $\mathbb{1}$ and its duals.

In BV10, we use this explicit description of the coend of $\mathcal{Z}(\mathcal{F})$ to show that the center $\mathcal{Z}(\mathcal{F})$ of a spherical fusion category $\mathcal{F}$ is modular. In particular, this implies that if $\mathcal{F}$ is a spherical fusion category of invertible dimension over an algebraic closed field $\mathbb{k}$, then $\mathcal{Z}(\mathcal{F})$ is a modular ribbon fusion category (this last result was first shown in [Mü03] using different methods).

Also, this description of the coend of $\mathcal{Z}(\mathcal{F})$ leads to an explicit algorithm (involving Hopf diagrams BV05]) for computing the Reshetikhin-Turaev invariants defined with $\mathcal{Z}(\mathcal{F})$. Moreover, this approach allows one to define these invariants over an arbitrary base ring, without assumption on the dimension of $\mathcal{F}$ (if the dimension of $\mathcal{F}$ is not invertible, this yields 'non-semisimple' invariants). See [BV08] for details. 


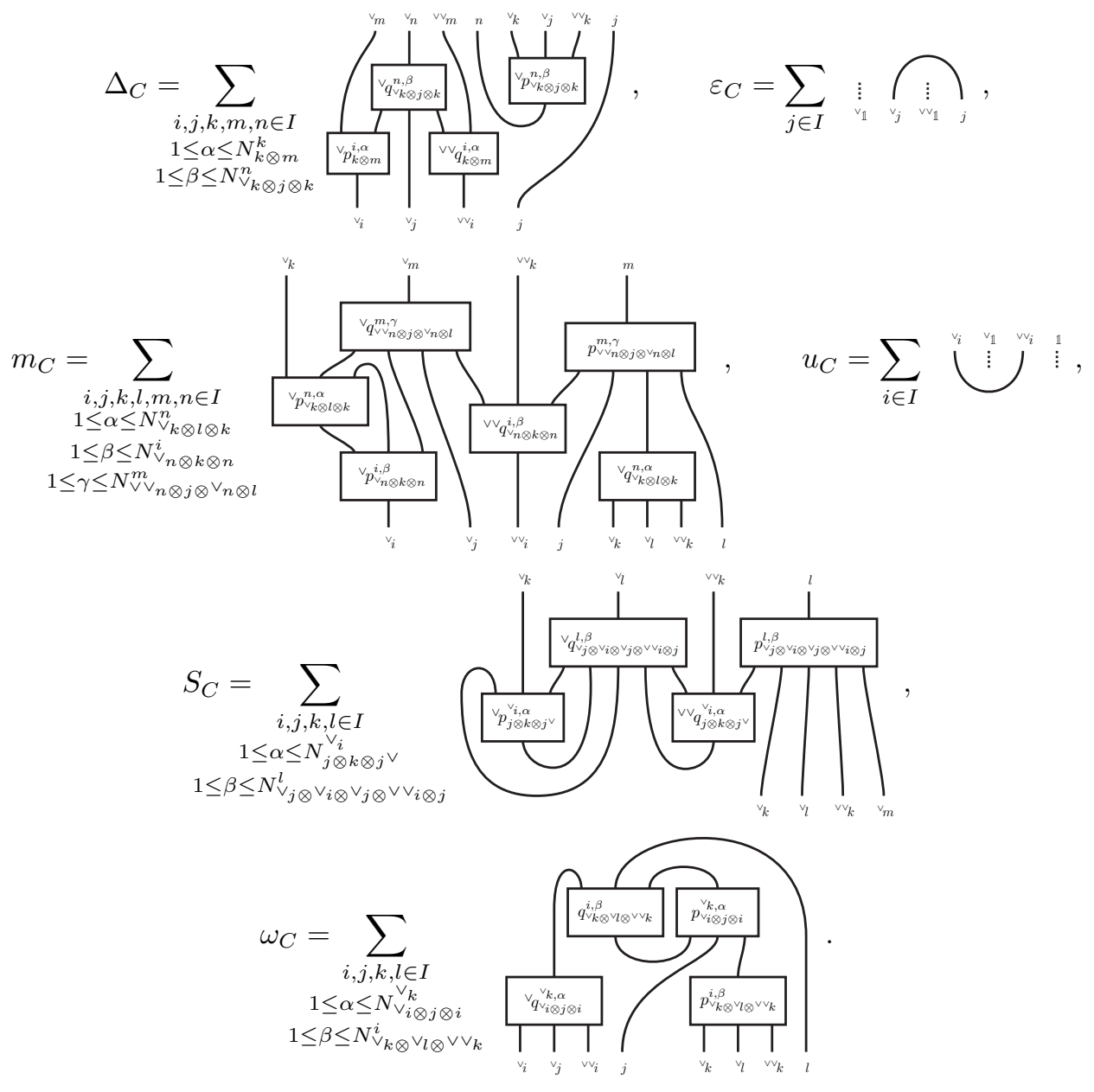

Figure 4. Structural morphisms of the coend of $\mathcal{Z}(\mathcal{F})$ 


\section{REFERENCES}

[Bec69] J. Beck, Distributive laws, Sem. on Triples and Categorical Homology Theory (ETH, Zürich, 1966/67), Springer, Berlin, 1969, pp. 119-140. MR0241502 (39:2842)

[Bes97] Y. Bespalov, Crossed modules and quantum groups in braided categories, App. Cat. Struc. 5 (1997), 155-204. MR1456522 (98k:18005)

[Bor94] F. Borceux, Handbook of categorical algebra. 2. Encyclopedia of Mathematics and its Applications, vol. 51, Cambridge University Press, Cambridge, 1994. MR 1313497 (96g:18001b)

[BV05] A. Bruguières and A. Virelizier, Hopf diagrams and quantum invariants, Algebr. Geom. Topol. 5 (2005), 1677-1710 (electronic). MR2186115 (2006k:57029)

[BV07] , Hopf monads, Advances in Math. 215 (2007), 679-733. MR2355605 (2009b:18006)

[BV08] , Categorical centers and Reshetikhin-Turaev invariants, Acta Math. Viet. 33 (2008). MR.2501845

[BV10] - On the center of fusion categories, in preparation.

[BW96] J. Barrett and B. Westbury, Invariants of piecewise-linear 3-manifolds, Trans. Amer. Math. Soc. 348 (1996), no. 10, 3997-4022. MR1357878 (97f:57017)

[Dri90] V. Drinfeld, On almost cocommutative Hopf algebras, Leningrad Math. J. 1 (1990), no. 2, 321-342. MR 1025154 (91b:16046)

[DS07] B. Day and R. Street, Centres of monoidal categories of functors, Categories in Algebra, Geometry and Mathematical Physics, Contemporary Mathematics, 431, 2007. MR2342829 (2008m:18011)

[ENO05] P. Etingof, D. Nikshych, and V. Ostrik, On fusion categories, Ann. of Math. 162 (2005), no. 2, 581-642. MR.2183279 (2006m:16051)

[Kas95] C. Kassel, Quantum Groups, Springer-Verlag, New York,1995. MR,1321145 (96e:17041)

[Lyu95] V. Lyubashenko, Invariants of 3-manifolds and projective representations of mapping class groups via quantum groups at roots of unity, Comm. Math. Phys. 172 (1995), no. 3, 467-516. MR.1354257 (97c:57018)

[Mü03] M. Müger, From subfactors to categories and topology. ii. The quantum double of tensor categories and subfactors, J. Pure Appl. Alg. 180 (2003), 159-219. MR1966525 (2004f:18014)

[Mac98] S. Mac Lane, Categories for the Working Mathematician, second ed., Springer-Verlag, New York, 1998. MR1712872(2001j:18001)

[Maj95] S. Majid, Foundations of quantum group theory, Cambridge Univ. Press, Cambridge, xix, 607 p., 1995. MR1381692 (97g:17016)

[Moe02] I. Moerdijk, Monads on tensor categories, J. Pure Appl. Algebra 168 (2002), no. 2-3, 189-208. MR1887157(2003e:18012)

[RT91] N. Reshetikhin and V. Turaev, Invariants of 3-manifolds via link polynomials and quantum groups, Invent. Math. 103 (1991), 547-597. MR.1091619 (92b:57024)

[Str72] R. Street, The formal theory of monads, J. Pure Appl. Algebra 348 (1972), no. 2, 149-168. MR0299653(45:8701)

[Tur94] V. Turaev, Quantum Invariants of Knots and 3-manifolds, Walter de Gruyter \& Co., Berlin, 1994. MR1292673(95k:57014)

Département de Mathématiques, Université Montpellier II, Place Eugène Bataillan, 34095 Montpellier Cedex 05, France

E-mail address: bruguier@math.univ-montp2.fr

Département de Mathématiques, Université Montpellier iI, Place Eugène Bataillan, 34095 Montpellier Cedex 05, France

E-mail address: virelizi@math.univ-montp2.fr 